\title{
Initial Investigation of Improved Volumetric Imaging of Concrete Using Advanced Processing Techniques
}

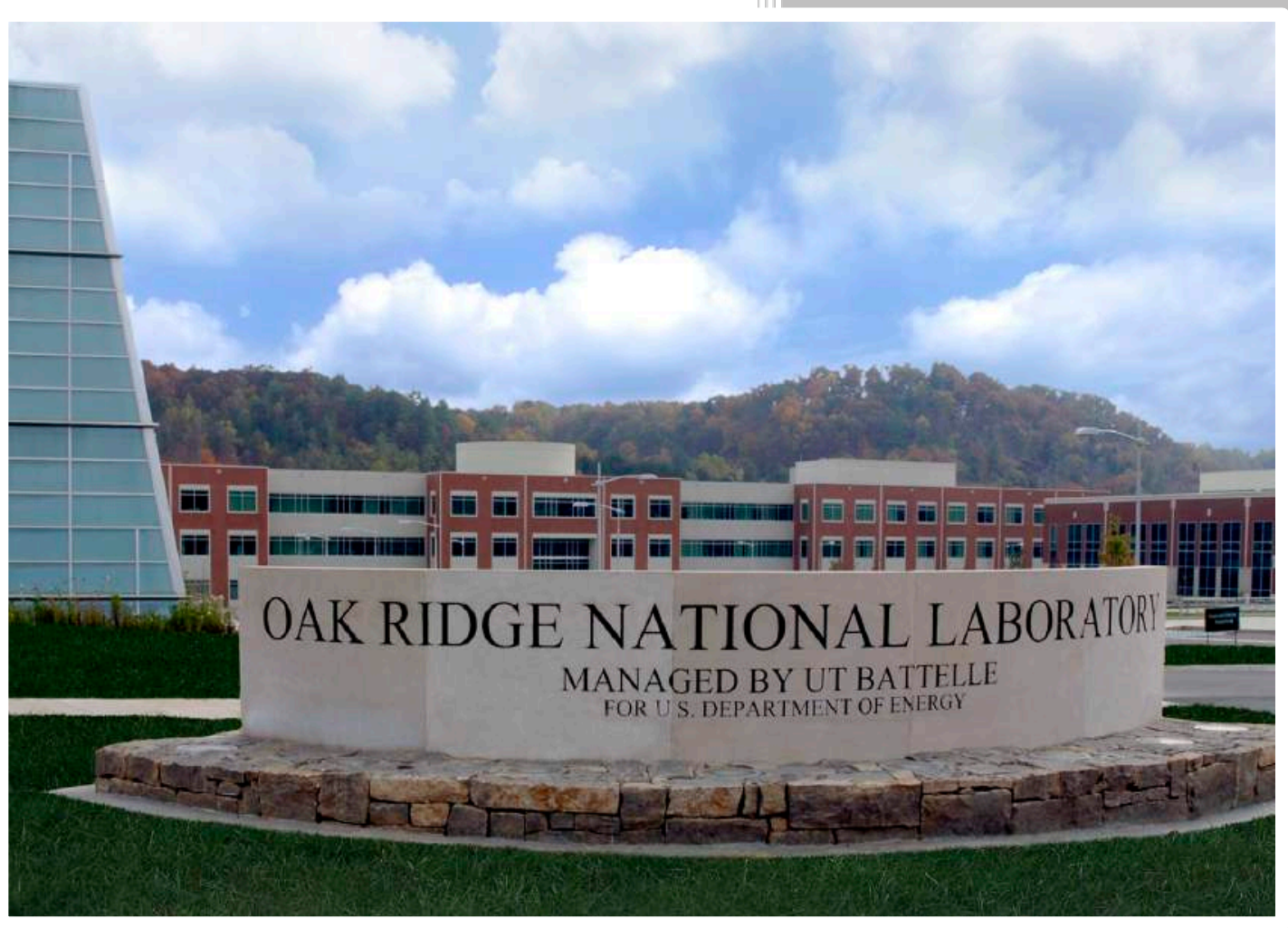

\section{Approved for public release.} Distribution is unlimited.
D. A. Clayton

A. P. Albright

H. J. Santos-Villalobos

September 2014 


\section{DOCUMENT AVAILABILITY}

Reports produced after January 1, 1996, are generally available free via US Department of Energy (DOE) SciTech Connect.

Website http://www.osti.gov/scitech/

Reports produced before January 1, 1996, may be purchased by members of the public from the following source:

National Technical Information Service

5285 Port Royal Road

Springfield, VA 22161

Telephone 703-605-6000 (1-800-553-6847)

TDD 703-487-4639

Fax 703-605-6900

E-mail info@ntis.gov

Website http://www.ntis.gov/help/ordermethods.aspx

Reports are available to DOE employees, DOE contractors, Energy Technology Data Exchange representatives, and International Nuclear Information System representatives from the following source:

Office of Scientific and Technical Information

PO Box 62

Oak Ridge, TN 37831

Telephone 865-576-8401

Fax 865-576-5728

E-mail reports@osti.gov

Website http://www.osti.gov/contact.html

This report was prepared as an account of work sponsored by an agency of the United States Government. Neither the United States Government nor any agency thereof, nor any of their employees, makes any warranty, express or implied, or assumes any legal liability or responsibility for the accuracy, completeness, or usefulness of any information, apparatus, product, or process disclosed, or represents that its use would not infringe privately owned rights. Reference herein to any specific commercial product, process, or service by trade name, trademark, manufacturer, or otherwise, does not necessarily constitute or imply its endorsement, recommendation, or favoring by the United States Government or any agency thereof. The views and opinions of authors expressed herein do not necessarily state or reflect those of the United States Government or any agency thereof. 
Light Water Reactor Sustainability Program

\section{INITIAL INVESTIGATION OF IMPROVED VOLUMETRIC IMAGING OF CONCRETE USING ADVANCED PROCESSING TECHNIQUES}

D. A. Clayton

A. P. Albright

H. J. Santos-Villalobos

Date Published:

September 2014

Prepared by

OAK RIDGE NATIONAL LABORATORY

Oak Ridge, Tennessee 37831-6283

managed by

UT-BATTELLE, LLC

for the

US DEPARTMENT OF ENERGY

under contract DE-AC05-00OR22725 



\section{CONTENTS}

Page

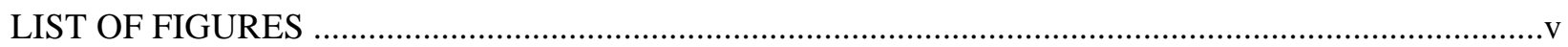

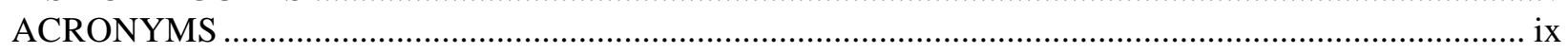

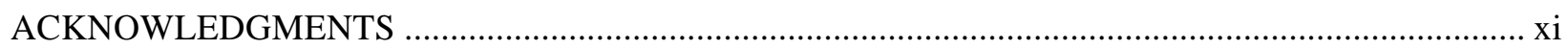

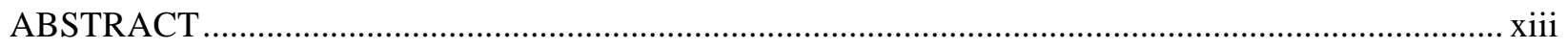

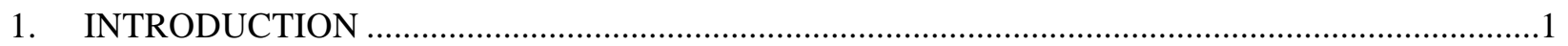

2. DEVELOPMENT OF THE FREQUENCY BANDING TECHNIQUE ........................................

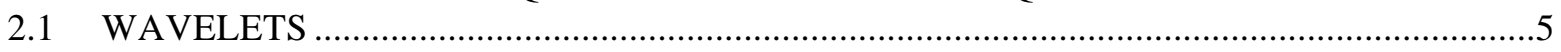

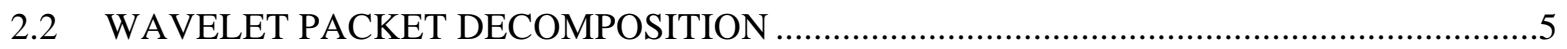

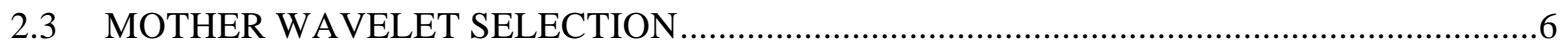

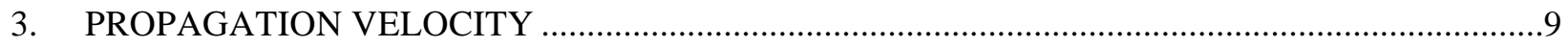

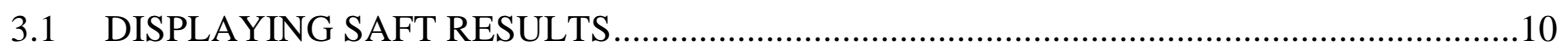

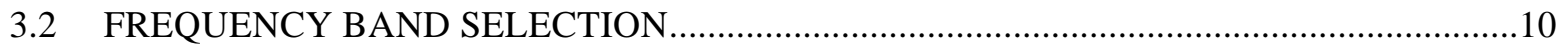

3.3 PROCEDURE OF THE FREQUENCY BANDING TECHNIQUE ...................................13

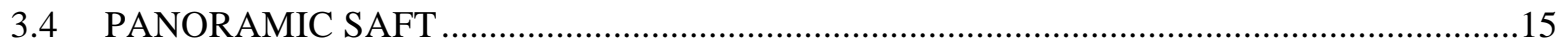

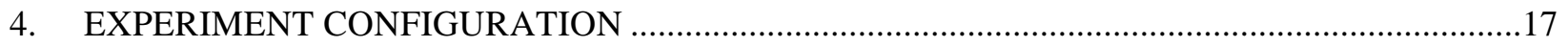

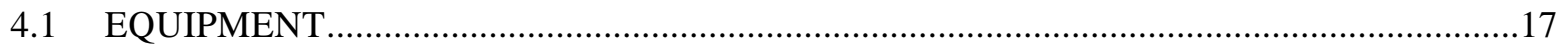

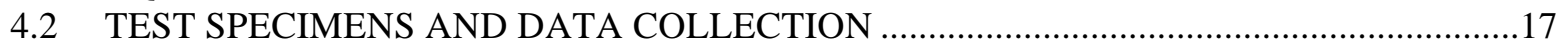

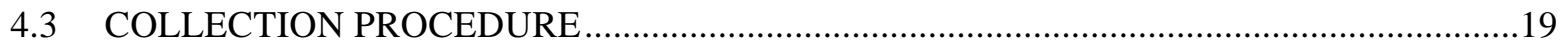

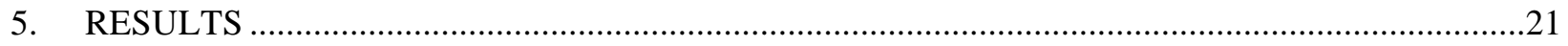

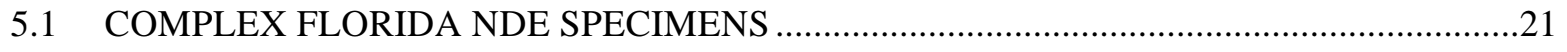

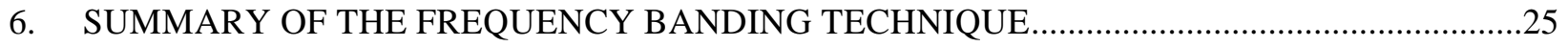

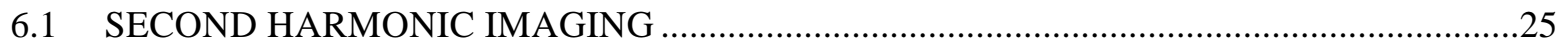

7. TOTAL FOCUSING METHOD - IMPLEMENTATION OF THE TOTAL FOCUSING

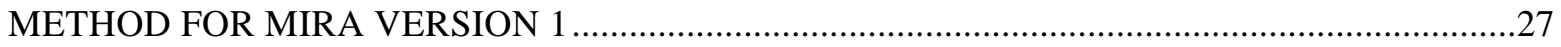

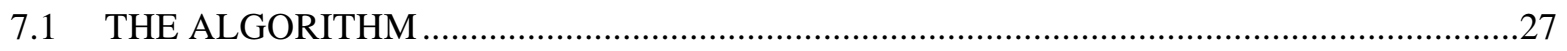

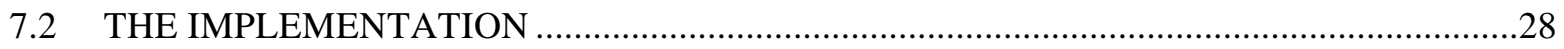

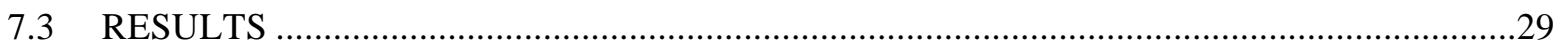

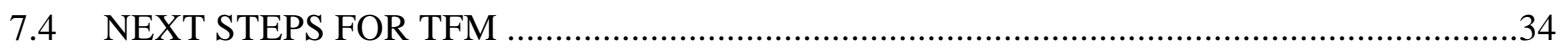

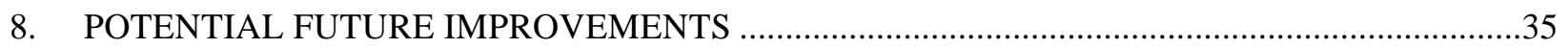

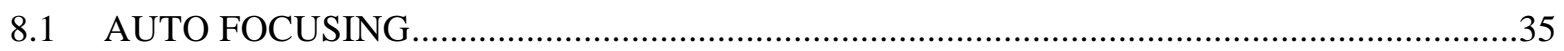

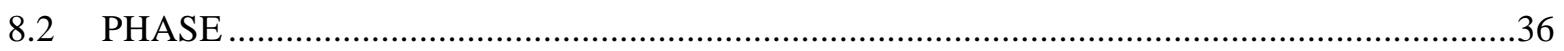

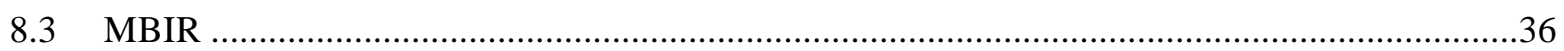

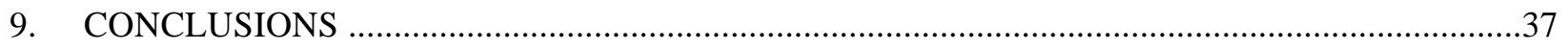

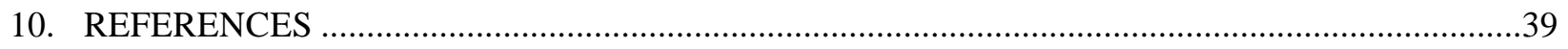

APPENDIX A. INTERPRETATION AND ANALYSIS OF ULTRASONIC LINEAR ARRAY

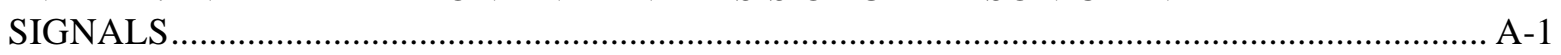

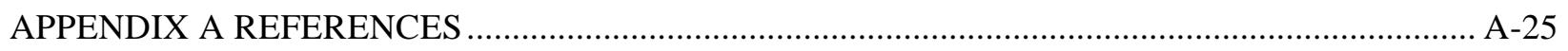

APPENDIX B. COMPLETE DETAILS OF FREQUENCY BAND SELECTION ............................... 



\section{LIST OF FIGURES}

Figure

Page

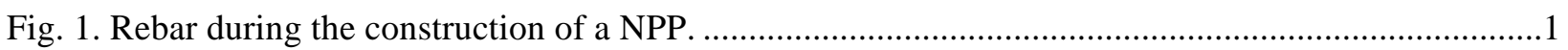

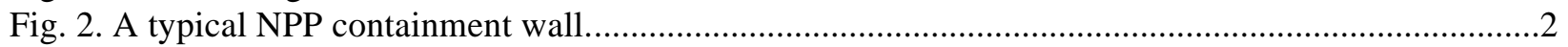

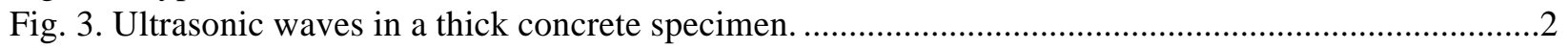

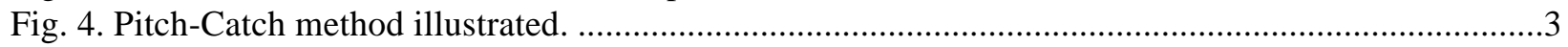

Fig. 5. Comparison of signal strength of transmitted shear waves from a $10 \mathrm{in.}$ test specimen..................4

Fig. 6. (a) Block diagram of a wavelet decomposition and (b) block diagram of a wavelet packet decomposition.

Fig. 7. Example of physical similarity between fundamental signal and the $\psi$ function of mother wavelet......

Fig. 8. A "good" energy segmentation in which nearly half the energy is in the $31-63 \mathrm{kHz}$ band.

Fig. 9. A "bad" energy segmentation in which only a quarter of the energy is in the $31-63 \mathrm{kHz}$

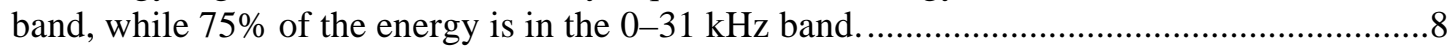

Fig. 10. (a) The SAFT results displayed as generated and (b) the SAFT results displayed after taking the absolute value of the Hilbert transform of the results. ............................................. 10

Fig. 11. Original data with a frequency range of $0 \sim 500 \mathrm{kHz}$ (bandwidth $500 \mathrm{kHz}$ )..........................11

Fig. 12. Data details - data: Node 15; frequency range: $0 \sim 31.25 \mathrm{kHz}$; bandwith: $31.25 \mathrm{kHz} . \ldots \ldots \ldots \ldots \ldots . .11$

Fig. 13. Data details - data: Node 16; frequency range: $31.25 \sim 62.5 \mathrm{kHz}$; bandwith: $31.25 \mathrm{kHz} . \ldots \ldots \ldots \ldots .12$

Fig. 14. Data details - data: Node 18; frequency range: $93.75 \sim 125 \mathrm{kHz}$; bandwith: $31.25 \mathrm{kHz}$............12

Fig. 15. Data details - data: Node 34; frequency range: $46.875 \sim 62.5 \mathrm{kHz}$; bandwidth: $15.625 \mathrm{kHz} . \ldots \ldots .12$

Fig. 16. Data details - data: Node 69; frequency range: $46.875 \sim 54.6875$; bandwidth: $7.8125 \mathrm{kHz} \ldots \ldots . .13$

Fig. 17. Data details - data: Node 139; frequency range: $46.875 \sim 50.78125$; bandwidth: 3.90625

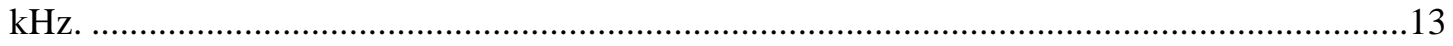

Fig. 18. Flowchart showing the typical work flow for frequency banding a dataset..................................14

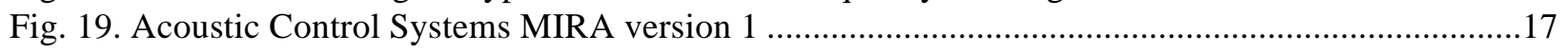

Fig. 20. Orientation and location of the rebar mats in the rebar test specimen........................................18

Fig. 21. Schematic of the honeycomb, crack, and rebar bonding defects contained in Specimen-6..........18

Fig. 22. Locations of each horizontal scan set and its positions. The red lines with arrowheads are

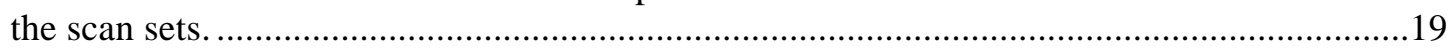

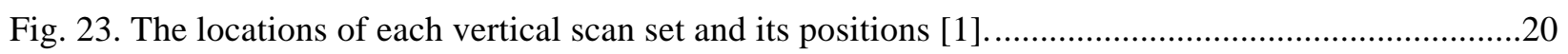

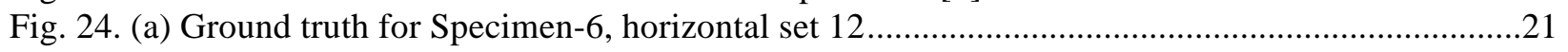

Fig. 25. The Panoramic SAFT B-scans of a vertical scan of Specimen-6, which passed over four

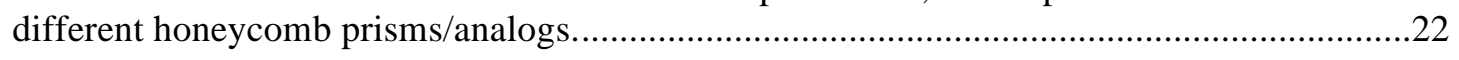

Fig. 26. Comparison between Panoramic SAFT B-scans with and without frequency banding.................23

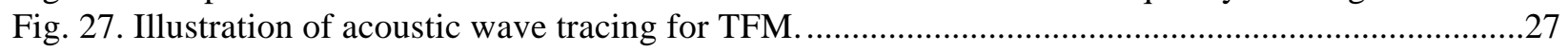

Fig. 28. (a) Illustration of divergence angle for transmitters and receivers and (b) plot of weight values as a function of divergence angle..........................................................................29

Fig. 29. Illustration of regions used for our quality tests: (a) contrast and noise and (b) PSF effects..........30

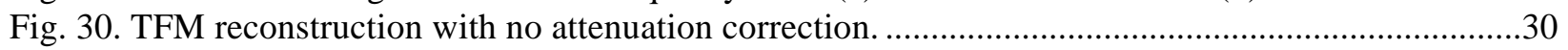

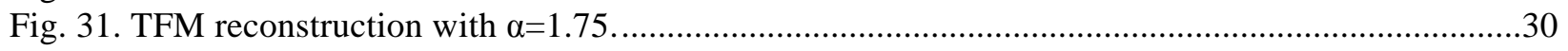

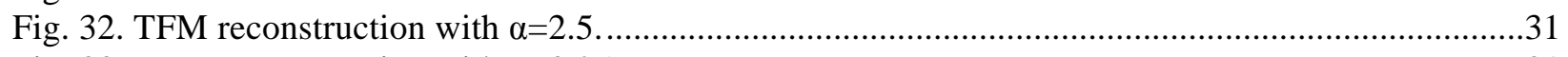

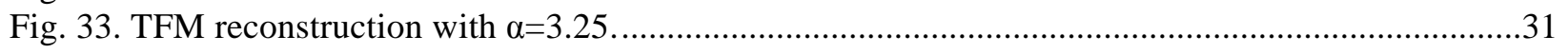

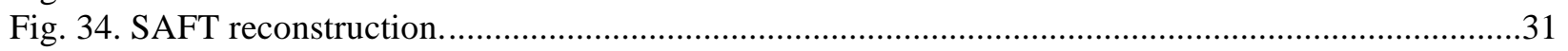

Fig. 35. Rebar line profiles assuming transducers in full contact with concrete surface. Results after

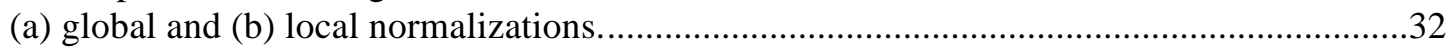

Fig. 36. Illustration of region used for local normalization. ...............................................................32 
Fig. 37. Normalized image reconstructions assuming a couplant thickness $d c=5 \mathrm{~mm}$. (a) TFM reconstruction with (a) linear and (b) non-linear $(\alpha=2.5)$ attenuation correction and (c) SAFT reconstruction.

Fig. 38. Rebar line profiles assuming a couplant gap of $5 \mathrm{~mm}$. Results after (a) global and (b) local normalizations.

Fig. A.1. Representation of potential contributing point sources at a constant time (Roundtrip) from the emitting/receiving transducer according to the fundamental expression. ....................... A-4

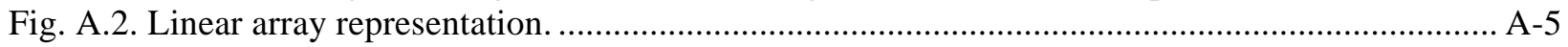

Fig. A.3. Schematic of the CRCP subsurface at the example scan location. ........................................ A-6

Fig. A.4. Example emitting-receiving pairs from an ultrasonic linear array scan................................ A-7

Fig. A.5. Example SAFT reconstruction with the apodization factor equal to (a) 1 and (b) defined

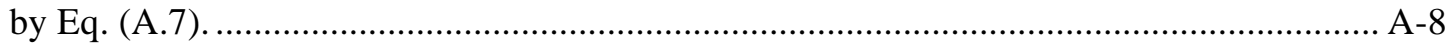

Fig. A.6. Determination of the direct arrival peak using the instantaneous amplitude envelope. .......... A-10

Fig. A.7. SAFT reconstruction and example column data................................................................. A-11

Fig. A.8. SAFT-IA reconstruction and example column data. ...................................................... A-12

Fig. A.9. Forensic verification of the focused reinforcement location within the SAFT-IA B-scan...... A-12

Fig. A.10. SAFT-IA B-scans from the cored location as well as 10 subsequent scans in the longitudinal direction.

A-13

Fig. A.11. Schematic representation of the process of creating SAFT-3D reconstructions. ................. A-14

Fig. A.12. SAFT 3D reconstruction using the SAFT-IA B-scan reconstructions shown in Fig. A.10... A-14

Fig. A.13. Example set of nine overlapping SAFT-IA B-scans used to create a SAFT Panoramic....... A-16

Fig. A.14. Five SAFT-Pan examples at a PCC joint.................................................................... A-17

Fig. A.15. Progression in identifying the centroid of reflections caused by round inclusions. .............. A-18

Fig. A.16. SAFT-Pan reconstruction with imprecise step size input............................................... A-20

Fig. A.17. Determination of overlapping regions between the reconstructions and determination of the next panoramic reconstruction............................................................................ A-21

Fig. A.18. Similarity of overlapping region curves used for placement of SAFT-IA reconstructions into the SAFT-EPan reconstruction. ........................................................................... A-23

Fig. A.19. Reconstruction of nine overlapping scans over three dowels to create (a) SAFT-Pan and (b) SAFT-EPan reconstructions.

Fig. B.1. Each node is labeled with its index number, the percentage of the total energy in the signal it contains, and the frequency range (rounded to nearest integer for readability)...................

Fig. B.2. Data details - data: original; frequency range: $0 \sim 500 \mathrm{kHz}$; bandwidth: $500 \mathrm{kHz} \ldots \ldots \ldots \ldots \ldots \ldots . . . . . . .4$

Fig. B.3. Data details - data: Node 15; frequency range: $0 \sim 31.25 \mathrm{kHz}$; bandwidth: $31.25 \mathrm{kHz} \ldots \ldots \ldots \ldots . . . \mathrm{B}-4$

Fig. B.4. Data details - data: Node 16; frequency range: $31.25 \sim 62.5 \mathrm{kHz}$; bandwidth: $31.25 \mathrm{kHz} \ldots \ldots . . \mathrm{B}-5$

Fig. B.5. Data details - data: Node 17; frequency range: $62.5 \sim 93.75 \mathrm{kHz}$; bandwidth: $31.25 \mathrm{kHz} \ldots \ldots \ldots . . \mathrm{B}-5$

Fig. B.6. Data details - data: Node 18; frequency range: $93.75 \sim 125 \mathrm{kHz}$; bandwidth: $31.25 \mathrm{kHz} \ldots \ldots \ldots . . .6-6$

Fig. B.7. Data details - data: Node 31; frequency range: $0 \sim 15.625 \mathrm{kHz}$; bandwidth: $15.625 \mathrm{kHz} \ldots \ldots . . . \mathrm{B}-6$

Fig. B.8. Data details - data: Node 32; frequency range: $15.625 \sim 31.25 \mathrm{kHz}$; bandwidth: 15.625

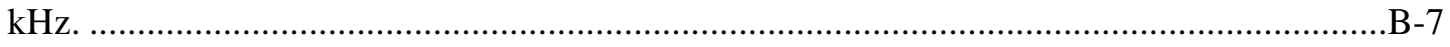

Fig. B.9. Data details - data: Node 33; frequency range: $31.25 \sim 46.875$ kHz; bandwidth: 15.625

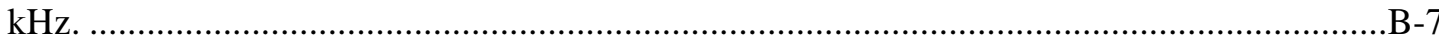

Fig. B.10. Data details - data: Node 34; frequency range: $46.875 \sim 62.5 \mathrm{kHz}$; bandwidth: 15.625

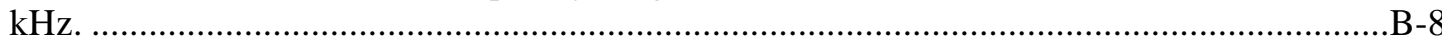

Fig. B.11. Data details - data: Node 37; frequency range: $93.75 \sim 109.375 \mathrm{kHz}$; bandwidth: 15.625

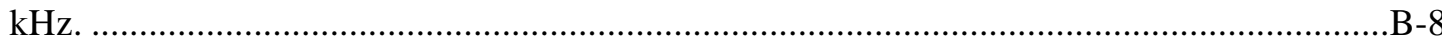

Fig. B.12. Data details - data: Node 38; frequency range: $109.375 \sim 125 \mathrm{kHz}$; bandwidth: 15.625

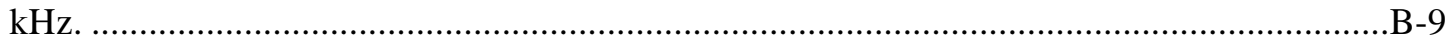

Fig. B.13. Data details - data: Node 63; frequency range: $0 \sim 7.8125 \mathrm{kHz}$; bandwidth: $7.8125 \mathrm{kHz} \ldots \ldots . . \mathrm{B}-9$ Fig. B.14. Data details - data: Node 64; frequency range: $7.8125 \sim 15.625 \mathrm{kHz}$; bandwidth: 7.8125 $\mathrm{kHz}$. 
Fig. B.15. Data details - data: Node 65; frequency range: $15.625 \sim 23.4375 \mathrm{kHz}$; bandwidth:

$7.8125 \mathrm{kHz}$.

Fig. B.16. Data details - data: Node 66; frequency range: $23.4375 \sim 31.25 \mathrm{kHz}$; bandwidth: 7.8125 $\mathrm{kHz}$.

Fig. B.17. Data details - data: Node 67; frequency range: $31.25 \sim 39.0625 \mathrm{kHz}$; bandwidth: 7.8125 $\mathrm{kHz}$

Fig. B.18. Data details - data: Node 68; frequency range: $39.0625 \sim 46.875 \mathrm{kHz}$; bandwidth: $7.8125 \mathrm{kHz}$.

Fig. B.19. Data details - data: Node 69; frequency range: $46.875 \sim 54.6875 \mathrm{kHz}$; bandwidth: $7.8125 \mathrm{kHz}$.

Fig. B.20. Data details - data: Node 70; frequency range: $54.6875 \sim 62.5 \mathrm{kHz}$; bandwidth: 7.8125 $\mathrm{kHz}$.

Fig. B.21. Data details - data: Node 75; frequency range: $93.75 \sim 101.5625 \mathrm{kHz}$; bandwidth: $7.8125 \mathrm{kHz}$.

Fig. B.22. Data details - data: Node 76; frequency range: $101.5625 \sim 109.375 \mathrm{kHz}$; bandwidth: $7.8125 \mathrm{kHz}$.

Fig. B.23. Data details - data: Node 139; frequency range: $46.875 \sim 50.78125 \mathrm{kHz}$; bandwidth: $3.90625 \mathrm{kHz}$.

Fig. B.24. Data details - data: Node 140; frequency range: $50.78125 \sim 54.6875 \mathrm{kHz}$; bandwidth: $3.90625 \mathrm{kHz}$.

Fig. B.25. Data details - data: Node 141; frequency range: $54.6875 \sim 58.59375 \mathrm{kHz}$; bandwidth: $3.90625 \mathrm{kHz}$.

Fig. B.26. Data details - data: Node 142; frequency range: $58.59375 \sim 62.5 \mathrm{kHz}$; bandwidth: 



\section{ACRONYMS}

$\begin{array}{ll}\text { ASTM } & \text { The American Society for Testing and Materials } \\ \text { CRCP } & \text { Reinforced Concrete Pavement } \\ \text { CWT } & \text { Continuous Wavelet Transform } \\ \text { DWT } & \text { Discrete Wavelet Transform } \\ \text { FMC } & \text { Full Matrix Capture } \\ \text { LWR } & \text { light water reactor } \\ \text { MBIR } & \text { Model-Based Image Reconstruction } \\ \text { NDE } & \text { Nondestructive Evaluation } \\ \text { NPPs } & \text { Nuclear Power Plants } \\ \text { PSF } & \text { Point Spread Function } \\ \text { ROI } & \text { Region of Interest } \\ \text { SAFT } & \text { Synthetic Aperture Focusing Technique } \\ \text { SAR } & \text { Synthetic Aperture Radar } \\ \text { TF } & \text { time-frequency } \\ \text { TFM } & \text { Total Focusing Method } \\ \text { ToF } & \text { time-of-flight }\end{array}$





\section{ACKNOWLEDGMENTS}

The authors at Oak Ridge National Laboratory would like to express our appreciation to our summer student research intern from North Carolina State University, Patrick Hoon. Using and building on his existing MATLAB skills, Patrick generated several thousand nondestructive evaluation images from ultrasonic array data previously collected. Several of these images appear in this report. 



\begin{abstract}
Materials issues are a key concern for the existing nuclear reactor fleet as material degradation can lead to increased maintenance, increased downtime, and increased risk. Extending reactor life to 60 years and beyond will likely increase susceptibility and severity of known forms of degradation. Additionally, new mechanisms of materials degradation are also possible. A multitude of concrete-based structures are typically part of a light water reactor (LWR) plant to provide foundation, support, shielding, and containment functions. Concrete has been used in the construction of Nuclear Power Plants (NPPs) because of three primary properties: its inexpensiveness, its structural strength, and its ability to shield radiation. Examples of concrete structures important to the safety of LWR plants include containment buildings, spent fuel pools, and cooling towers. This use has made its long-term performance crucial for the safe operation of commercial NPPs. With respect to the concrete structures, age-related degradation may affect engineering properties, structural resistance/capacity, failure mode, and location of failure initiation that in turn may affect the ability of a structure to withstand challenges in service. To ensure the safe operation of NPPs, it is essential that the effects of potential degradation of the plant structures, as well as systems and components, be assessed and managed during both the current operating license period as well as subsequent license renewal periods. In contrast to many mechanical and electrical components, replacing many concrete structures is impractical. Therefore, it is necessary that safety issues related to plant aging and continued service of the concrete structures are resolved through sound scientific and engineering understanding.

Unlike most metallic materials, reinforced concrete is a nonhomogeneous material; a composite with a low-density matrix, reinforced concrete is a mixture of cement, sand, aggregate and water, and with a high-density reinforcement (typically 5\% in NPP containment structures) consisting of steel rebar or tendons. This heterogeneous nature increases the complexity of performing nondestructive evaluations (NDE) by adding "noise" to ultrasonic volumetric images.

This report examines the benefits of using time-frequency analysis with Synthetic Aperture Focusing Technique (SAFT). SAFT is an image reconstruction technique commonly used in conjunction with ultrasonic arrays. By using wavelet packet decomposition, the original ultrasound signals are decomposed into various frequency bands. Selected frequency bands are then reconstructed back into a time-series dataset. This facilitates highly selective analysis of the signal's frequency content and can be visualized using the familiar and reliable SAFT image reconstruction algorithm. Additionally, this paper briefly examines the benefits of using the Total Focusing Method (TFM) as compared to SAFT and explores possible benefits from Model Based Image Reconstruction (MBIR).
\end{abstract}





\section{INTRODUCTION}

Extending reactor life to 60 years and beyond will likely increase the reactor's susceptibility to and the severity of known forms of materials degradation. With respect to the concrete structures, age-related degradation may affect engineering properties, structural resistance/capacity, failure mode, and location of failure initiation that in turn may affect the ability of a structure to withstand challenges in service. In order to ensure the safe operation of Nuclear Power Plants (NPPs), it is essential that the effects of potential degradation of the plant structures, as well as systems and components, be assessed and managed during both the current operating license period as well as subsequent license renewal periods. In contrast to many mechanical and electrical components, replacement of many concrete structures is impractical. Therefore it is necessary that safety issues related to plant aging and continued service of the concrete structures are resolved through sound scientific and engineering understanding.

Unlike most metallic materials, reinforced concrete is a nonhomogeneous material; a composite with a low-density matrix, a mixture of cement, sand, aggregate and water, and a high-density reinforcement (typically 5\% in NPP containment structures) made up of steel rebar or tendons. Figure 1 illustrates the vast amount of rebar that appears in a typical NPP. Plants have been typically built with local cement and aggregate fulfilling the design specification regarding strength, workability, and durability, but as a consequence, each plant's concrete composition is unique and complex.

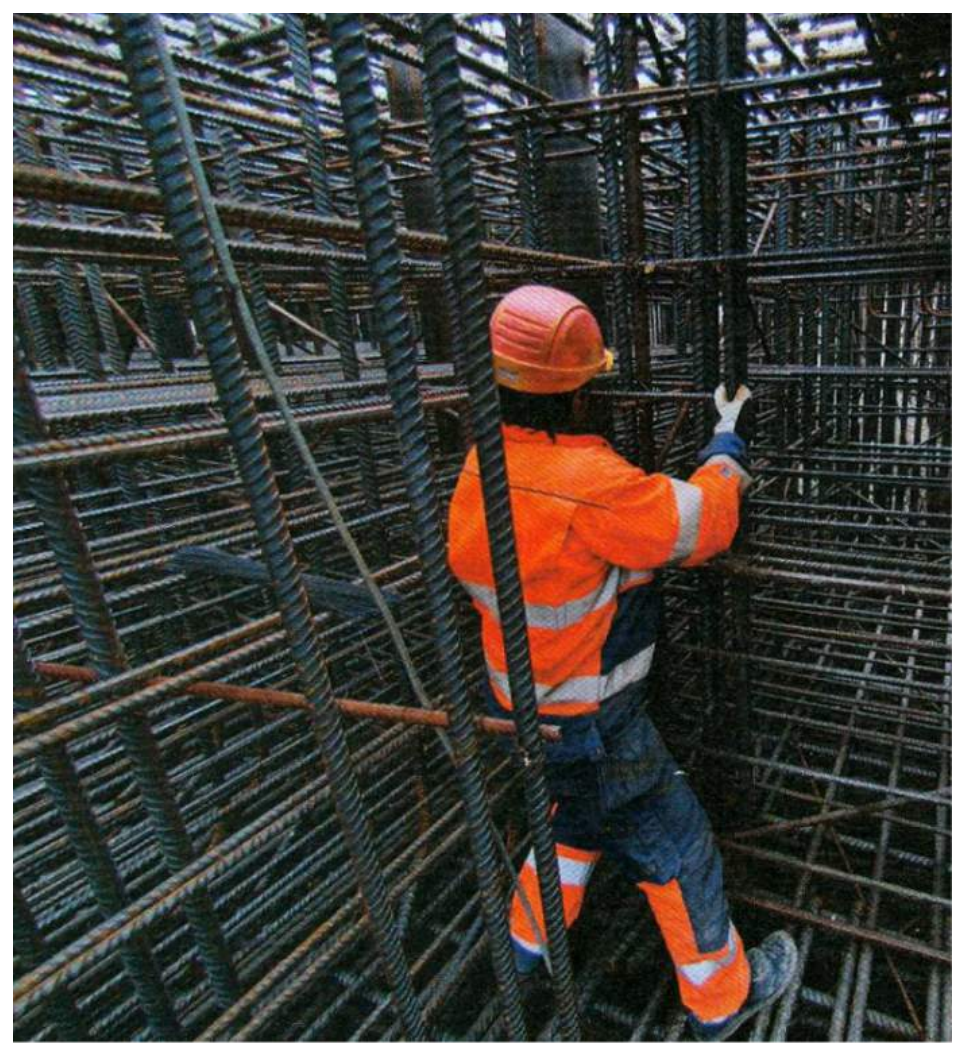

Fig. 1. Rebar during the construction of a NPP.

NPP concrete structures are often inaccessible, containing large volumes and massively thick concrete sections that are exposed to different environments (moisture, temperature) and a variety of degradation mechanisms (high temperatures, radiation exposure, chemical reactions) at different plant sites, all of which add to the complexity of determining the integrity/quality of the concrete. Often only one side of the concrete structure is readily available or one side has a steel liner, as illustrated in Fig. 2. Since the aspect ratio is so different from typical transportation concrete structures due to the thickness of concrete 
sections, additional reflections from lateral boundaries add to the complexity of received ultrasonic signals. Figure 3 illustrates the multiple ultrasonic reflections that are possible when the source is near a lateral boundary.

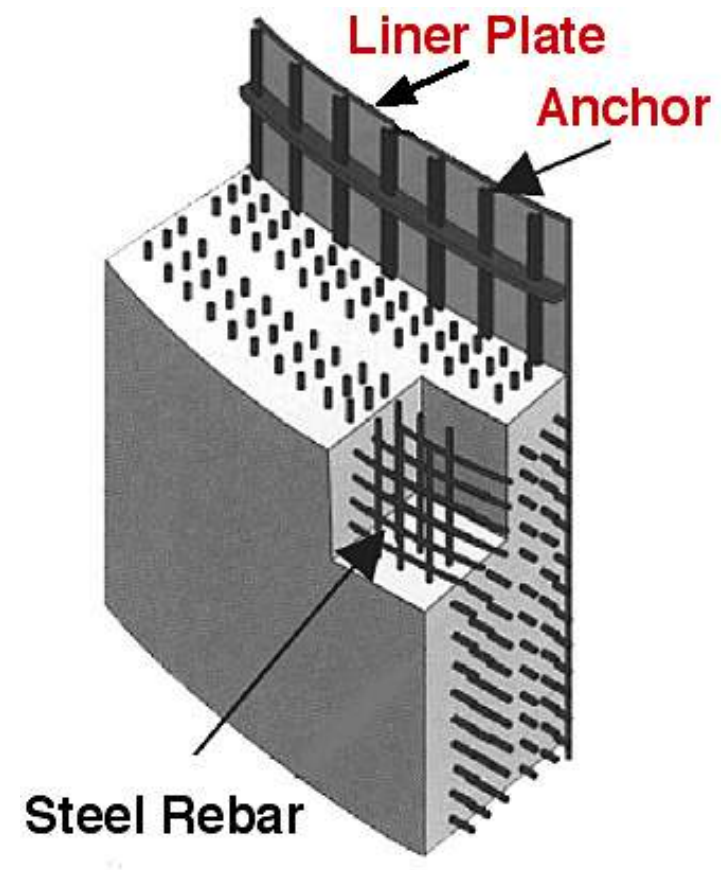

Fig. 2. A typical NPP containment wall.
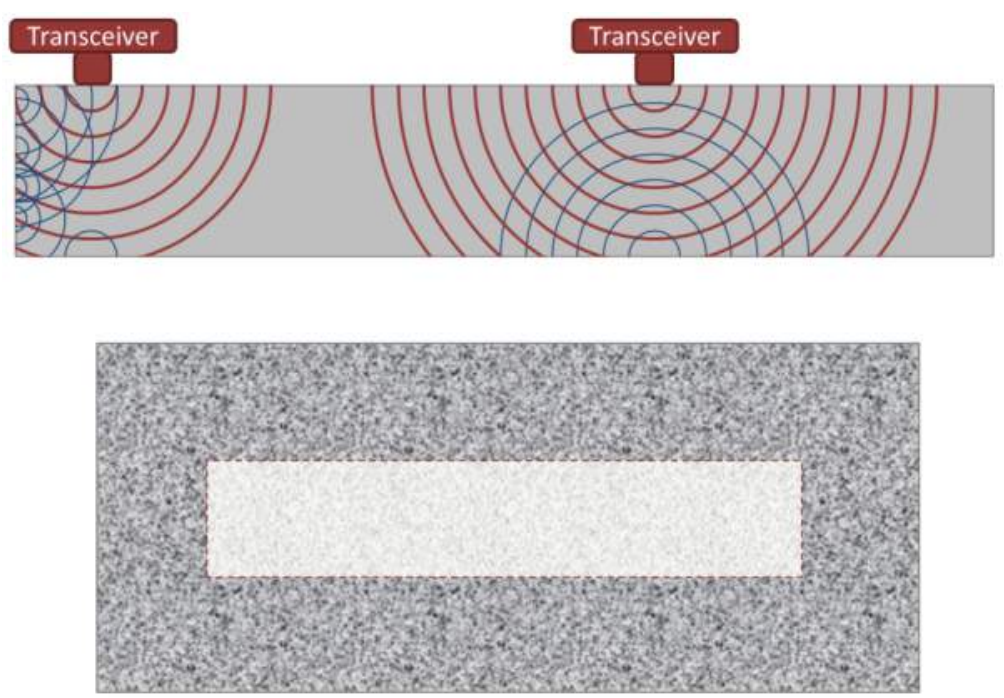

Fig. 3. Ultrasonic waves in a thick concrete specimen.

In ORNL/TM-2013/430 [1], seven different nondestructive evaluation (NDE) technologies were compared using two concrete test specimens from the Florida Department of Transportation's NDE Validation Facility in Gainesville, Florida. As documented in that report, ultrasonic linear array devices with 40 or more transducers performed best at volumetric imaging. These devices are based on the "pitchcatch" method of sending and receiving shear wave impulses at the surface, requiring only one-sided access and receiving the echoes at the original surface. Figure 4 illustrates how the "pitch-catch" method examines the volume under the instrument. 


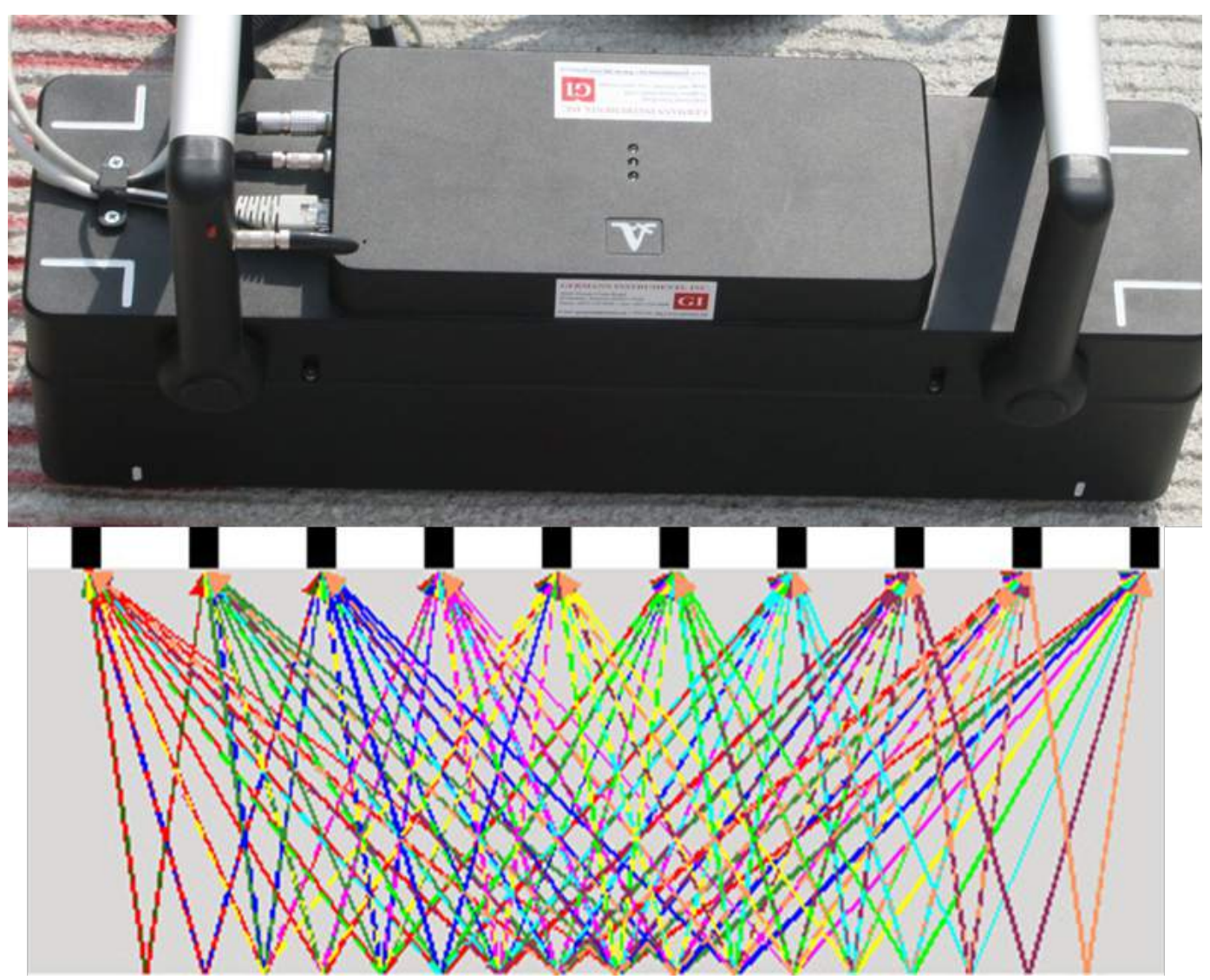

Fig. 4. Pitch-Catch method illustrated.

Improvements in transducer coupling technology have increased productivity by eliminating the need for application of a coupling agent to transfer the vibration to the concrete. The transducers have been developed to transmit and receive shear wave impulses, which allows for measurement pairs with multiple angles of transmission and reception at reduced transducer spacing for high-precision shear wave impulse measurements and eliminates the need for a manual mechanical impact. The redundancy and spatial diversity of the measurements provide an opportunity to use the Kirchoff migration-based focusing to create cross sections of the subsurface structure that correlate to the physical location of the internal concrete structure.

The additional thickness of concrete in NPP applications drastically decreases the signal-to-noise ratio on returned ultrasound signals since the signals must travel through the concrete twice so the echo can be received and analyzed. For the 10 in. thick test specimens examined in ORNL/TM-2013/430 [1], this reduction in signal strength is shown in Fig. 5. This reduction in signal to noise necessitates the development of advance signal processing techniques so NPP concrete structures, which may be a meter or more thick, can be examined. 


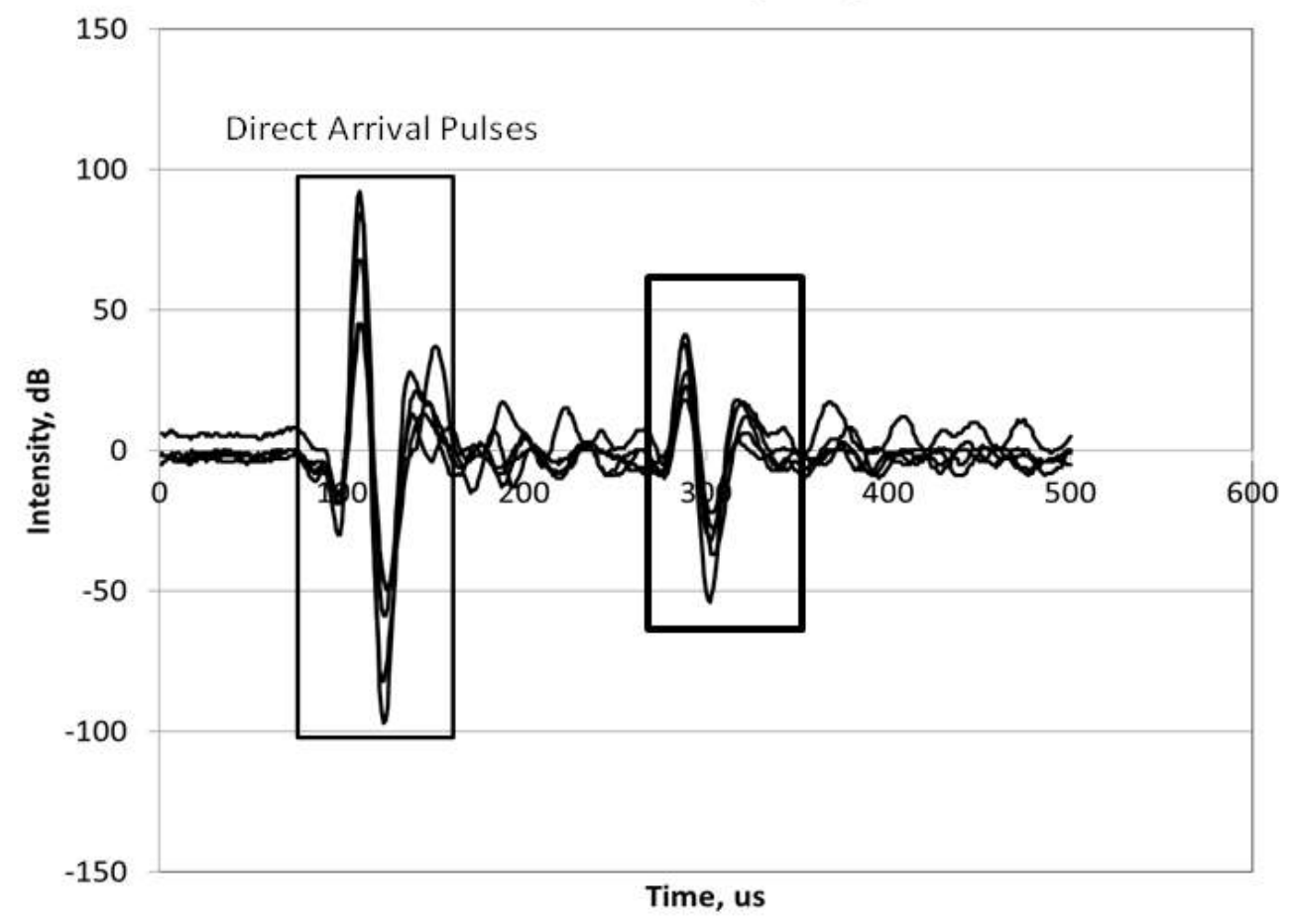

Fig. 5. Comparison of signal strength of transmitted shear waves from a $10 \mathrm{in}$. test specimen.

While a test specimen more typical of a NPP concrete structure is being designed and built (expected to be completed in early FY 2015), the test specimens from the Florida Department of Transportation's NDE Validation Facility serve as a good baseline comparison of methods. This report examines the benefits of using time-frequency analysis with Synthetic Aperture Focusing Technique (SAFT). SAFT is an image reconstruction technique commonly used in conjunction with ultrasonic arrays. By using wavelet packet decomposition, the original ultrasound signals are decomposed into various frequency bands. Selected frequency bands are then reconstructed back into a time-series dataset. This facilitates highly selective analysis of the signal's frequency content and can be visualized using the familiar and reliable SAFT image reconstruction algorithm. Additionally, this paper briefly examines the benefits of using the Total Focusing Method (TFM) as compared to SAFT and explores possible benefits from Model Based Image Reconstruction (MBIR). 


\section{DEVELOPMENT OF THE FREQUENCY BANDING TECHNIQUE}

The Time Frequency (TF) technique forming the core of our approach is the wavelet packet decomposition. However, instead of directly using the coefficients from the wavelet packet decomposition, we decompose the original received ultrasound signals into various frequency bands (nodes), select a node based on the frequency band it contains, and then reconstruct the node back to a time-series dataset with the same duration and sampling rate as the original signal. However, this reconstructed dataset contains only the specific band of frequencies contained in the reconstructed node. This process takes advantage of the reconstruction property of the mother wavelet, and the natural frequency segmentation provided by the continued decomposition of the previous decomposition results' nodes. Any decomposition node can be reconstructed. The end effect is a highly selective analysis of the received signal's frequency content which can be visualized using the familiar and reliable SAFT image reconstruction algorithm.

\subsection{WAVELETS}

Far more in-depth and detailed introductions to wavelets and multi-resolution analysis can be found in numerous publications such as [2], [3], and [4]. The basic terminology used in this paper is reviewed below.

The mother wavelet, also known as a basis function, is scaled and translated and then "compared" (i.e., convolved) with the signal being analyzed as shown in Eq. (1), where $a$ is the scale factor, $\tau$ is the translation parameter, $t$ is time, $x$ is the signal, and $\psi$ is the mother wavelet [2].

$$
C W T(a, \tau)=\int x(t) \Psi_{a \tau}(t) d t
$$

Using the multi-resolution analysis approach, each scaling and translation of the mother wavelet is convolved with the signal being analyzed, resulting in a decomposition of the analyzed signal into two nodes. These two decomposition nodes each contain half the bandwidth of the signal that was decomposed. The node containing the lower half of the original bandwidth is called the approximation. The node containing the upper half of the bandwidth is called the detail. Detail nodes and approximation nodes can be further decomposed. The results of an individual node's decomposition create two more nodes. One node contains the upper half of the decomposed node's bandwidth, while the other contains the lower half of the decomposed node's bandwidth. A graphical representation of this is shown in Fig. 6.

When the scale and translation parameters are swept across a continuous range, this convolution is known as the Continuous Wavelet Transform (CWT). Similarly, the Discrete Wavelet Transform (DWT) is the "adjustment" of the scale and translation parameters in discrete steps [4]. We perform a selective CWT packet decomposition.

\subsection{WAVELET PACKET DECOMPOSITION}

The motivation behind the use of wavelet packet decomposition over wavelet decomposition is based on the greater level of selectivity provided by wavelet packet decomposition. When performing a wavelet decomposition, only the approximation portion is decomposed by each successive decomposition as shown in Fig. 6(a). For wavelet packet decompositions, any approximation or detail can be decomposed, which Daubechies terms as the "splitting trick [4]," and is illustrated in Fig. 6(b). This allows a greater range of frequency bands and bandwidths to be selected and analyzed. Wavelet packet decomposition has the same advantageous properties as wavelet decomposition. The most important properties to this application are the low-frequency leakage and exact reconstruction properties. 


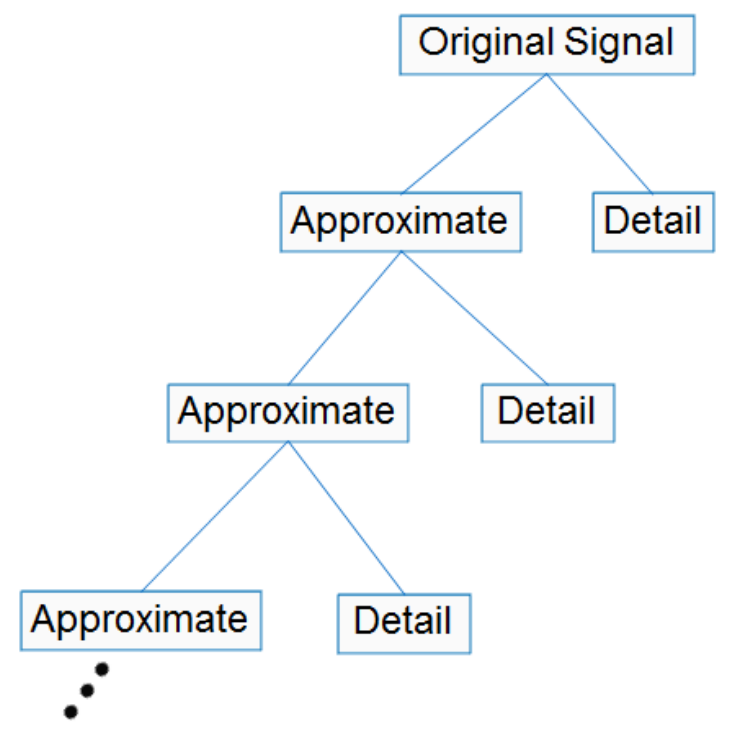

(a)

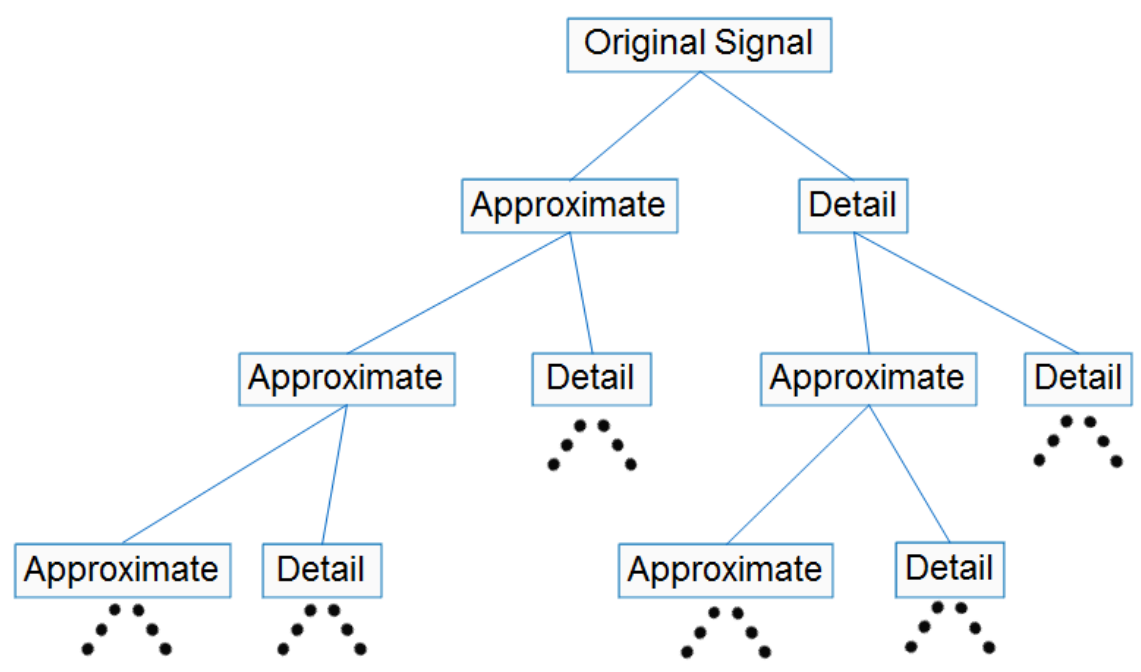

(b)

Fig. 6. (a) Block diagram of a wavelet decomposition and (b) block diagram of a wavelet packet decomposition.

\subsection{MOTHER WAVELET SELECTION}

One of the more challenging aspects of the wavelet decomposition, packet or otherwise, is the selection of the mother wavelet. The selection of a mother wavelet is outside the scope of this report, but listed below are a few general recommendations that proved useful in our selection experiments.

A sampling of individual channels collected using an ultrasonic linear array system from a known sound concrete sample should be analyzed first. Channels were chosen, which included at least one transmitter and receiver pairing that are side-by-side and one pairing where the transmitting and receiving transducers are on the extreme opposite edges of the linear array. The selection of a mother wavelet was then made using the following general rules of thumb. 
1. Physical Similarity - A mother wavelet whose shape generally resembles the "fundamental" shape of the signal is one of the simplest places to start. Ideally, the comparison would be made to the ultrasonic pulse coming directly from the transmitting transducer in a perfect situation (i.e., directly coupled to a receiving transducer). However, if this is not available, the closest equivalent should be used. Having access only to the data collected from a linear array instrument and not the actual instrument itself, the closest equivalent was the initial portion of the received signal. This signal contained the surface propagating wave front, which is what should be used in the comparison. Figure 7 shows an example of this comparison. Figure 7(a) shows three different received signals with $40 \mathrm{~mm}$ spacing between transmitter and receiver. Figure 7(b) shows the Symlet-7 mother wavelet.

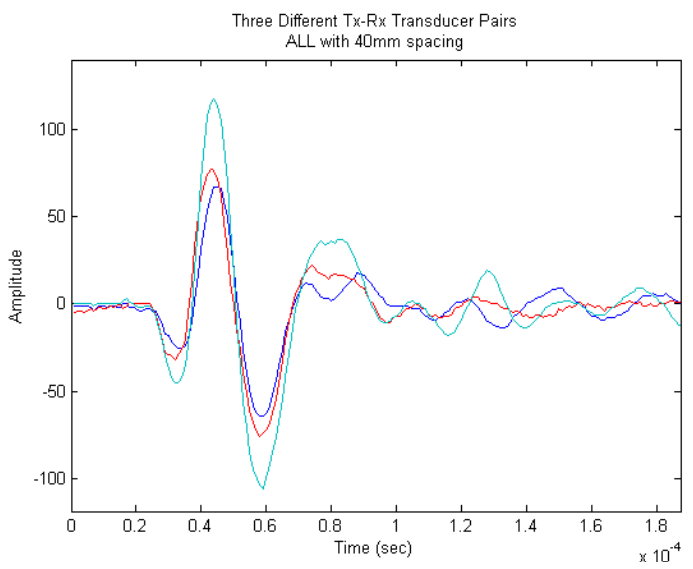

(a)

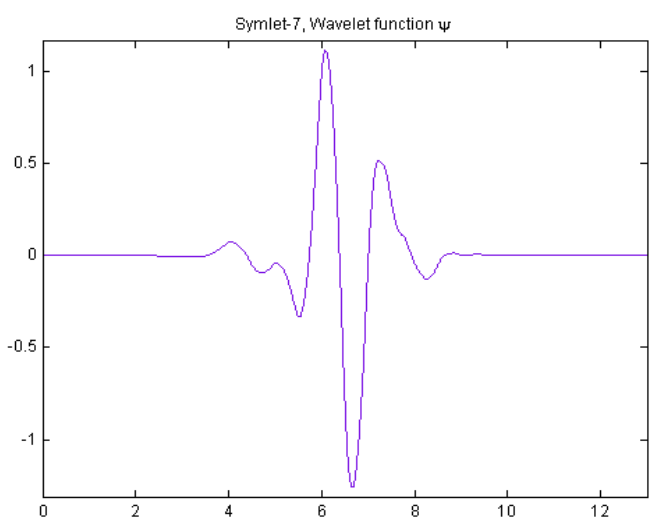

(b)

Fig. 7. Example of physical similarity between fundamental signal and the $\psi$ function of mother wavelet.

2. Energy Segmentation - If information on the excitation is available, specifically the nominal center frequency, then the quality of the frequency banding can be evaluated based on the amount of energy segmented into a band. For the instrument used to collect our data, the nominal center frequency is $50 \mathrm{kHz}$. When selecting the mother wavelet for our data, the percentage of energy segmented into the bands containing $50 \mathrm{kHz}$ were examined. In one case, the mother wavelet that appeared to have close physical similarities segmented the majority of the energy into a frequency band whose upper edge was nearly $20 \mathrm{kHz}$ less than the nominal center frequency. Figure 8 shows an example in which the mother wavelet has segmented the energy where expected. The nominal center frequency of the ultrasonic impulse was $50 \mathrm{kHz}$. Therefore, it is expected that the energy content of nodes containing the nominal center frequency should be non-trivial. Figure 9 illustrates the aforementioned situation in which the majority of the energy is well below where the system is known to emit it. This rule of thumb is only applicable in situations where the excitation frequency of the energy source (i.e., instrument) is known. Keep in mind, at this time the effect of various intrinsic concrete properties, defects, and the internal structure on frequency is unclear.

3. Compression - the level of compression a mother wavelet provides for the data can be used as an additional decision criterion. The goal is to retain as much energy as possible, using as few coefficients as possible.

Using the mother wavelet candidates chosen, similar individual channels from a dataset were collected that directly straddled a 1.5 in. steel dowel embedded in a known sound concrete sample at mid- 
depth (center of dowel at 6 in. in a 12 in. thick block). If little to no difference between the rules-of-thumb characteristics in the sound concrete block versus the rules-of-thumb characteristics in sound concrete block plus steel dowel were observed, the mother wavelet was considered to be a "good" selection for use on datasets collected using the same model and version of the ultrasonic linear array system.

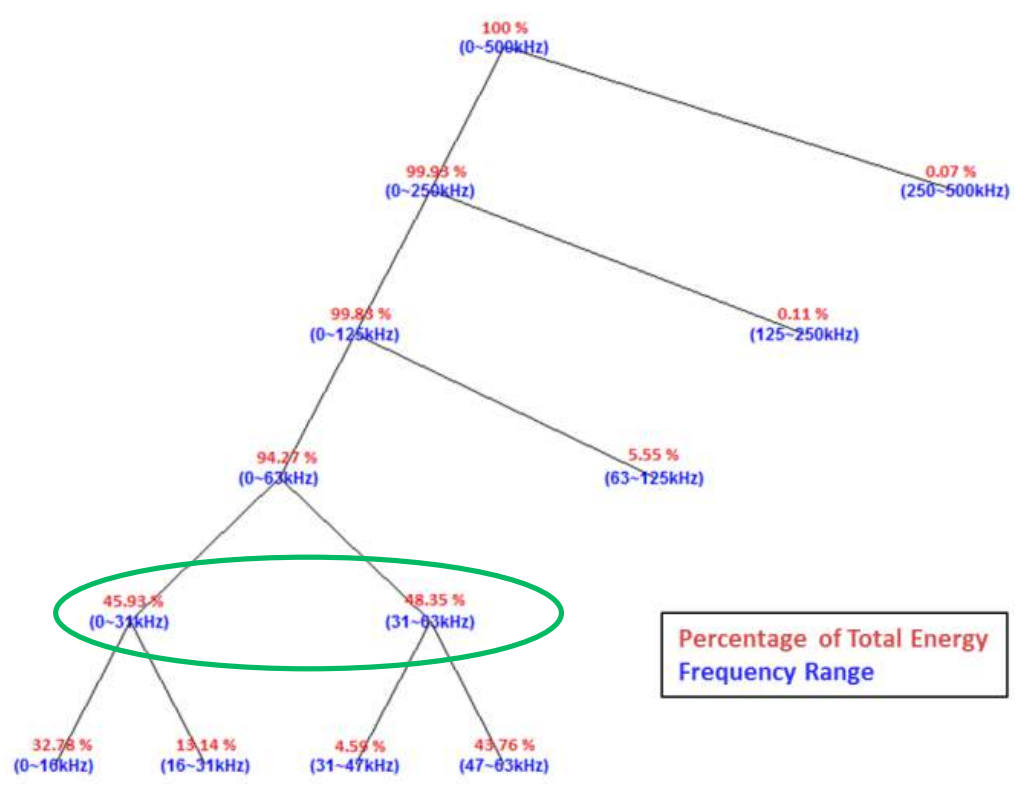

Fig. 8. A "good" energy segmentation in which nearly half the energy is in the $31-63 \mathrm{kHz}$ band.

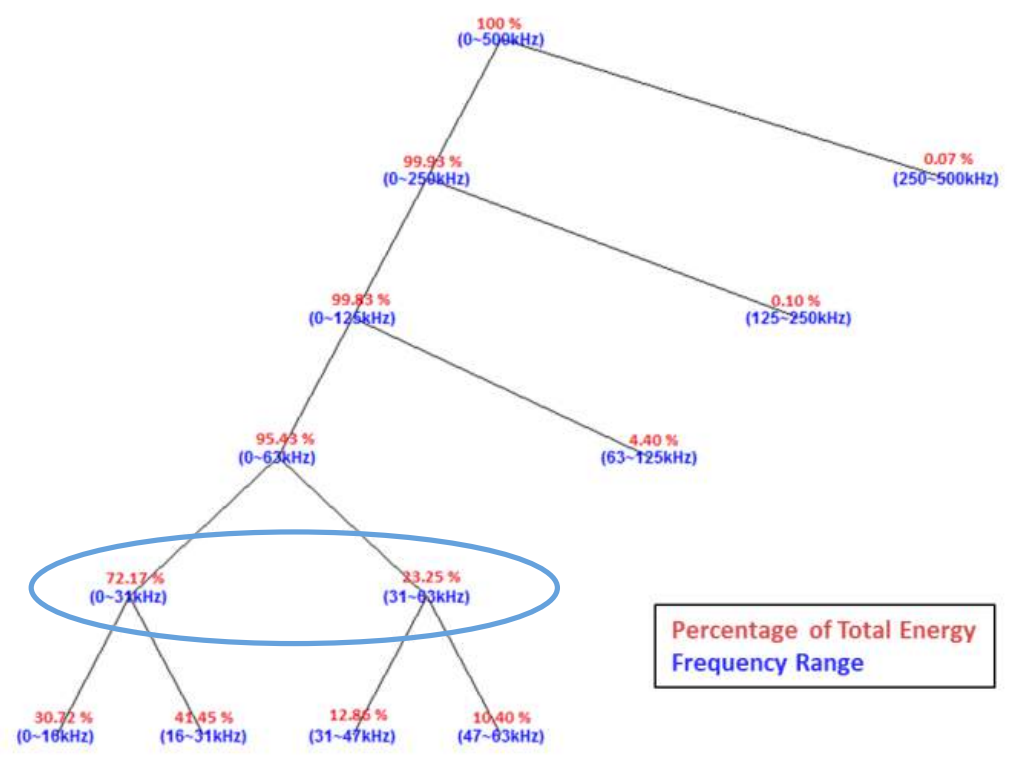

Fig. 9. A "bad" energy segmentation in which only a quarter of the energy is in the $31-63 \mathrm{kHz}$ band, while $75 \%$ of the energy is in the $0-31 \mathrm{kHz}$ band. 


\section{PROPAGATION VELOCITY}

The implementation of several image reconstruction algorithms specifically targeted for use with ultrasonic linear arrays has made the key role that the propagation velocity plays even more apparent. Appendix A contains additional information on the interpretation and analysis of ultrasonic linear array signals. It is crucial to keep in mind that concrete is a nonhomogeneous material in its composition; therefore, its corresponding wave velocity is not necessarily constant. The American Society for Testing and Materials (ASTM) has published a standard, ASTM C597, establishing a procedure and equations for calculating the propagation velocity of longitudinal waves in concrete [5]. The procedure suggests two different formulas for calculating velocity. In Eq. (2), the velocity, $V$, is dependent on knowing both the dynamic modulus of elasticity, $E$, the density, $\rho$, and the dynamic Poisson's ratio, $\mu$, of the concrete.

$$
V=\sqrt{\frac{E(1-\mu)}{\rho(1+\mu)(1-2 \mu)}} .
$$

The standard's purpose is to evaluate the quality of a concrete structure by comparing the expected velocity calculated by using Eq. (2) with a velocity measured using the time-of-flight (ToF) of an ultrasonic wave or by comparing the velocities calculated using Eq.(3) as the concrete structure ages. Equation (3) uses ToF of an ultrasonic wave to calculate the velocity.

$$
V=L / T,
$$

where the distance between the centers of the ultrasonic transducers is $L$ and the ToF is $T$.

However, the ASTM C597 standard is as much a list of problems and a warning regarding the calculation of the velocity using the ultrasonic ToF method as it is a standard. A few items mentioned that may cause the resulting propagation velocity to vary are

1. the water content of the concrete,

2. the thickness of the concrete,

3. poor coupling between the concrete surface and the transducers,

4. errors in the measurement of the spacing between the ultrasonic transducers,

5. the inability to operate in a through-transmission configuration,

6. the instrument operator,

7. proximity to steel (rebar), and

8. cracking and deterioration.

In fact, between different instruments and operators, up to a $20 \%$ variation is reported between the velocity calculations when in the presence of cracks and/or deterioration [5]. Aside from the differences in homogeneity, another challenge to calculating propagation velocity is that the modulus of elasticity for concrete can change significantly as a function of time, unlike homogeneous materials whose properties are usually the same over time. It has become increasingly apparent that an accurate method for determining the propagation velocity of an ultrasonic wave in large, thick, complex structures, such as nuclear reactor containment structures, requires investigation.

Ultimately, errors in the calculated or measured propagation velocity do not prevent the inspection of large, complicated structures. The errors result in inaccurate estimates of the depth that a defect or piece of rebar was detected. A method of modeling the ultrasonic waves in a large concrete object, where the effects of frequency, rebar density, and multi-pour boundaries can be examined, is needed to improve both detection and image construction. 


\subsection{DISPLAYING SAFT RESULTS}

The results from a SAFT reconstruction is a two-dimensional matrix of pixels. The value of each pixel is the summation of all the amplitude samples corresponding to a specific distance. This distance is determined using the estimated propagation velocity of the medium, calculated or measured, and the sampling rate of the acquisition system. The sampling rate provides the amount of time between each sample and the total amount of time from the start of the acquisition until a sample is acquired. This time multiplied by the propagation velocity yields the distance traveled in that amount of time. This is where the accuracy of the propagation velocity becomes critical. The sampling rate of the system provides ToF information. The ToF multiplied by the propagation velocity yields the total straight-line distance that was traveled corresponding to a sample. This distance traveled could be either a direct reflection or a multi-path reflection involving multiple reflections.

The resulting pixel values can be positive or negative. When colorized for visualization as an image, these positive and negative values yield an image, as shown in Fig. 10(a). The other, more commonly used, representation displays the envelope of the SAFT results. The envelope is calculated by taking the absolute value of the Hilbert transform of the SAFT results. This results in an image such as the one shown in Fig. 10(b). The SAFT results are for a dataset collected from a 12 in. thick sound concrete specimen containing a 1.5 in. diameter steel dowel rod at mid-depth (center of dowel at center of thickness) using a MIRA version 1. The MIRA was positioned such that the center of the array was straddling the dowel rod.

Typically, the envelope of the SAFT results is used due to the ease of defect identification thanks to the clear contrast. However, the envelope process has a downside that must be watched for. For example, if there are several closely spaced rebar elements, the envelope is likely to consolidate these into a single bright spot. Likewise, if there is a defect or rebar in close proximity to the back wall, the envelope almost always causes these defects to be "absorbed" by the back wall reflection, the end result being that it is often beneficial to examine SAFT results using both methods. In most cases, the enveloped version will prove to be sufficient by itself.

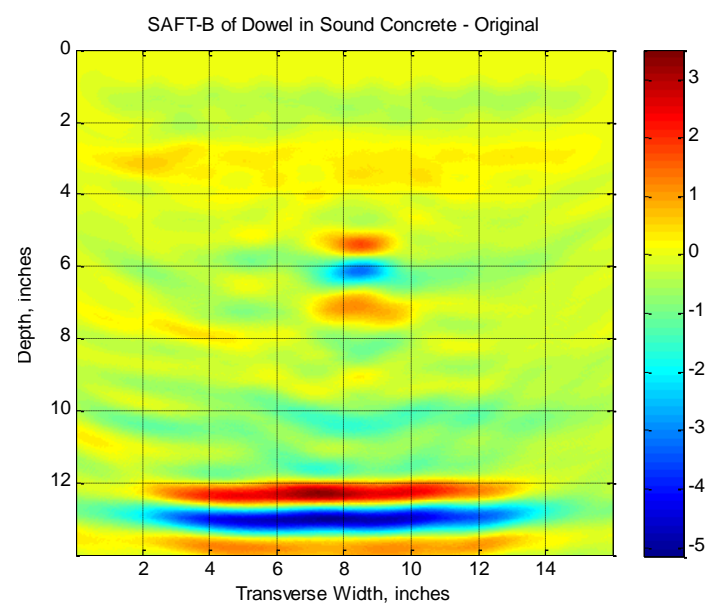

(a)

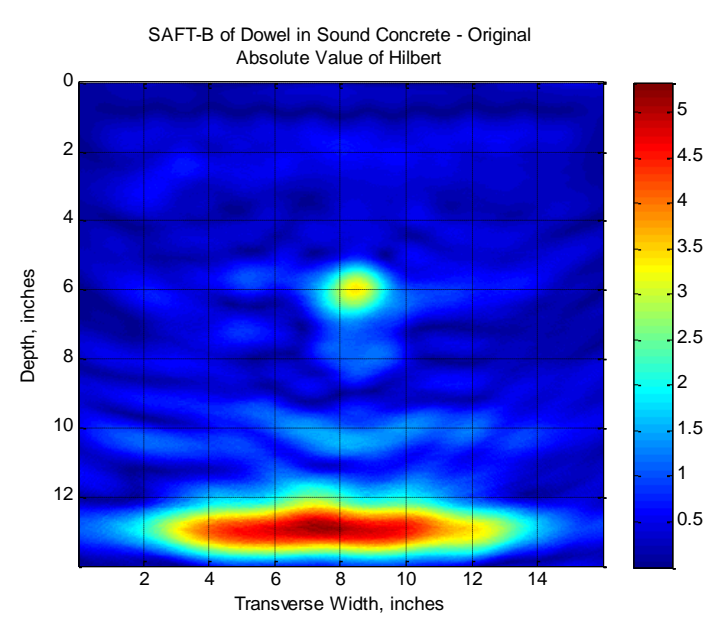

(b)

Fig. 10. (a) The SAFT results displayed as generated and (b) the SAFT results displayed after taking the absolute value of the Hilbert transform of the results.

\subsection{FREQUENCY BAND SELECTION}

Once a mother wavelet is chosen, the next decisions involve selecting which specific frequency bands are worth segmenting, reconstructing, and running SAFT on. 
Several of the better frequency bands considered while refining the frequency banding techniques are shown in Figs. 11-17. These frequency-banded SAFT results were evaluated on a 12 in. thick sound concrete specimen with a $1.5 \mathrm{in}$. dowel at mid-depth. The number range of the color bar on each image can be treated as an indicator of the energy and therefore information contained in a frequency band.

Figure 17 contains several illustrative examples showing frequency bands that proved to be worthwhile. Both the SAFT results as well as the envelope of the SAFT results are shown side-by-side. Both formats shown assist in conveying the information contained in each frequency band.

Some frequency bands are of no use because the frequency band contained has little energy. This can occur at both wide and narrow bandwidths. However, it is most pronounced in narrow frequency bands. A general feel for how much energy a particular frequency band's SAFT results contain can be assessed from the numeric range of each image's color bar. A complete display of all the frequency bands examined from the sound concrete specimen containing the $1.5 \mathrm{in}$. dowel during the development of the frequency banding technique can be found in Appendix B. Complete Details of Frequency Band Selection.
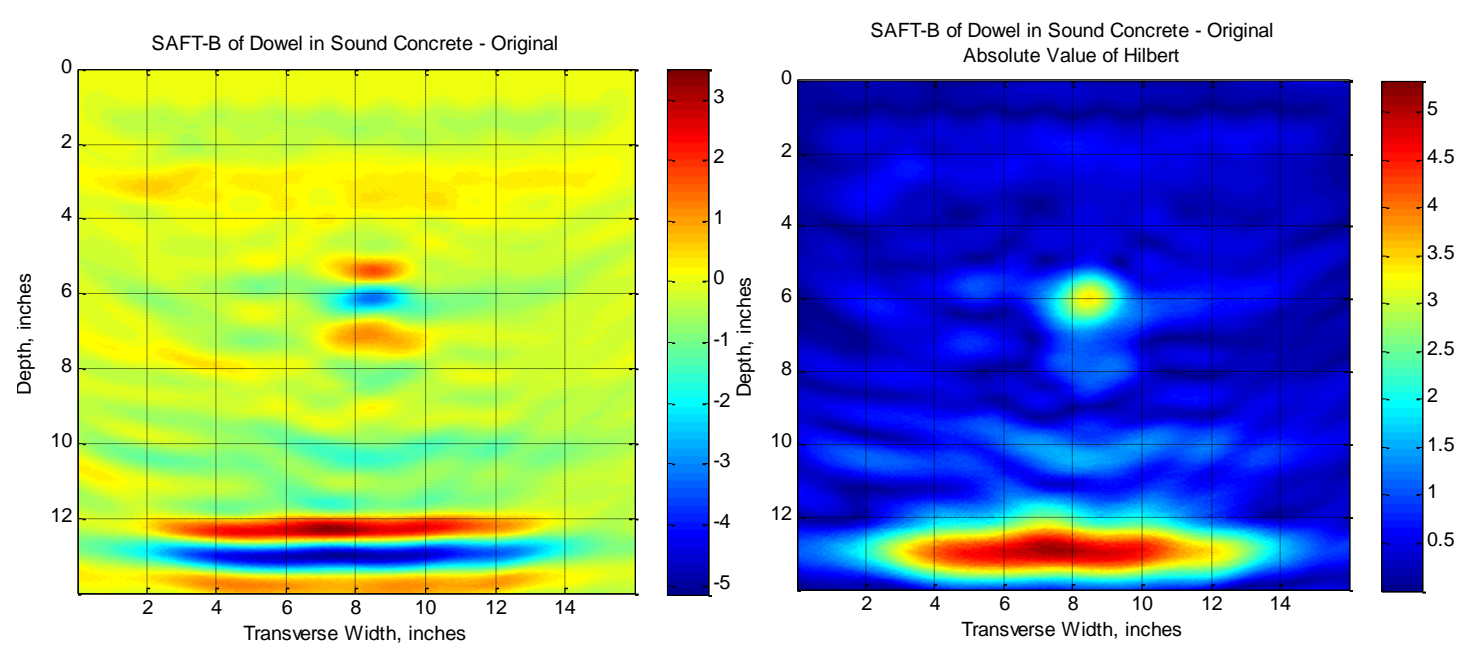

Fig. 11. Original data with a frequency range of $0 \sim 500 \mathrm{kHz}$ (bandwidth $500 \mathrm{kHz}$ ).
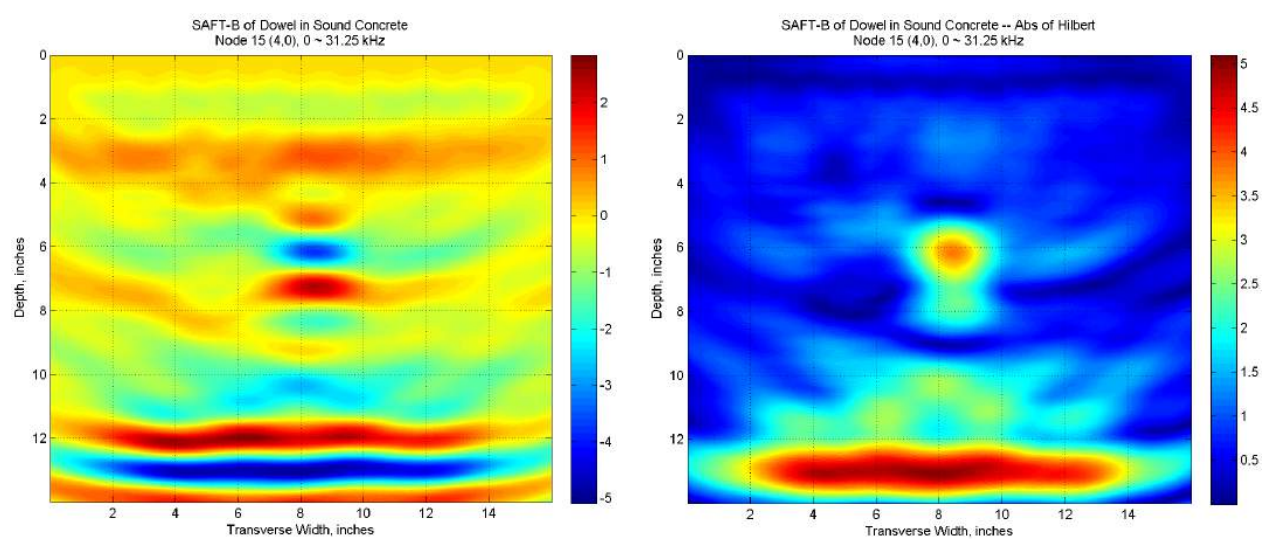

Fig. 12. Data details - data: Node 15; frequency range: $0 \sim 31.25 \mathrm{kHz}$; bandwith: $31.25 \mathrm{kHz}$. 

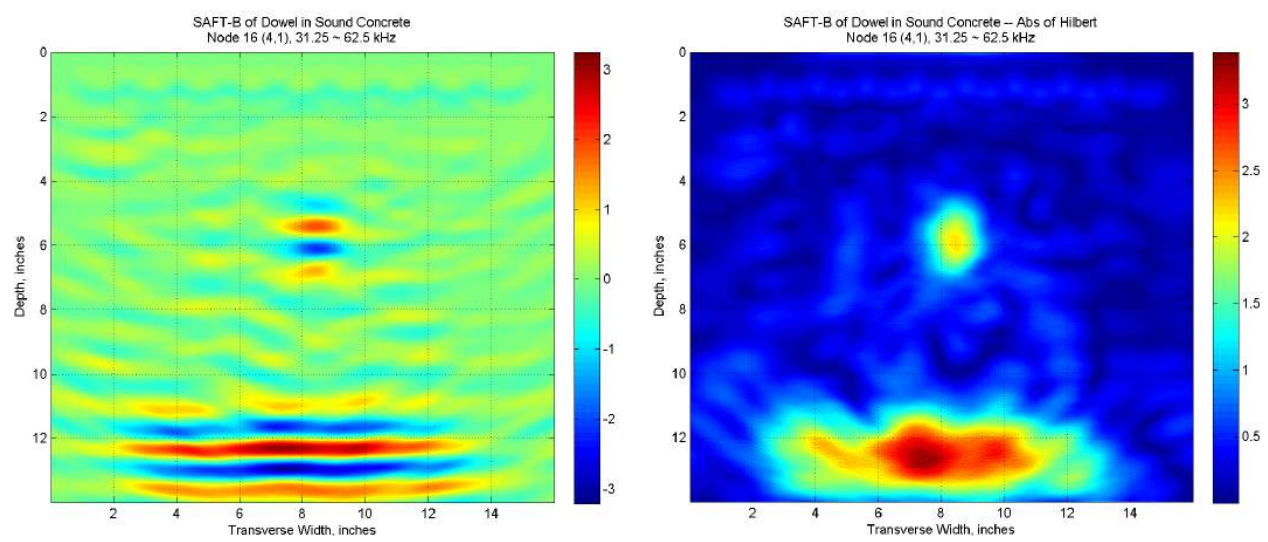

Fig. 13. Data details - data: Node 16; frequency range: $31.25 \sim 62.5 \mathrm{kHz}$; bandwith: $31.25 \mathrm{kHz}$.
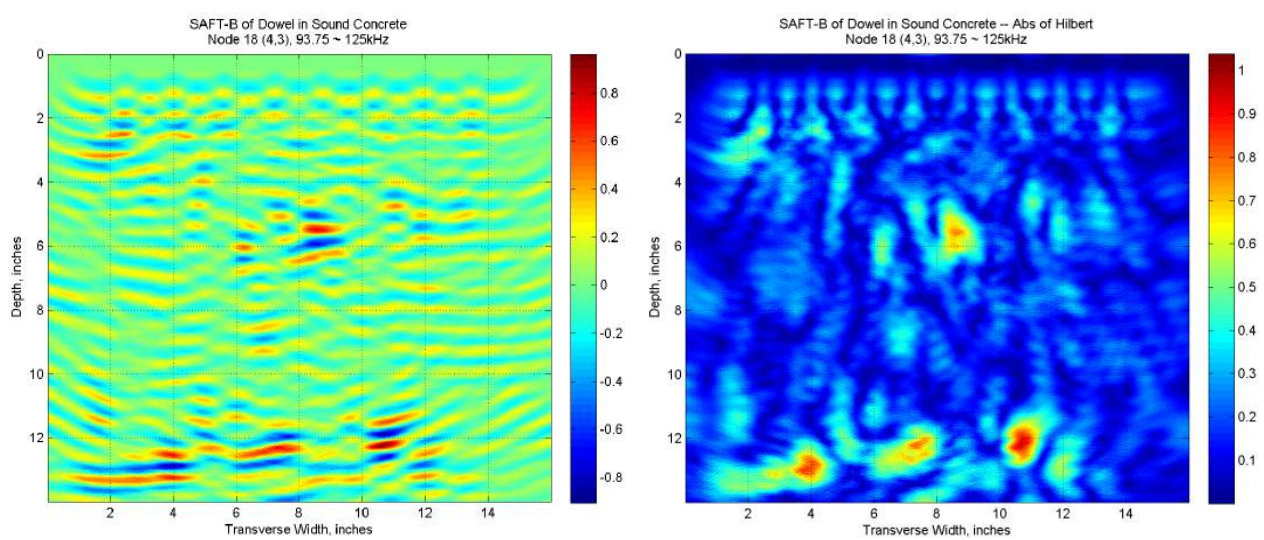

Fig. 14. Data details - data: Node 18; frequency range: $93.75 \sim 125$ kHz; bandwith: 31.25 kHz.
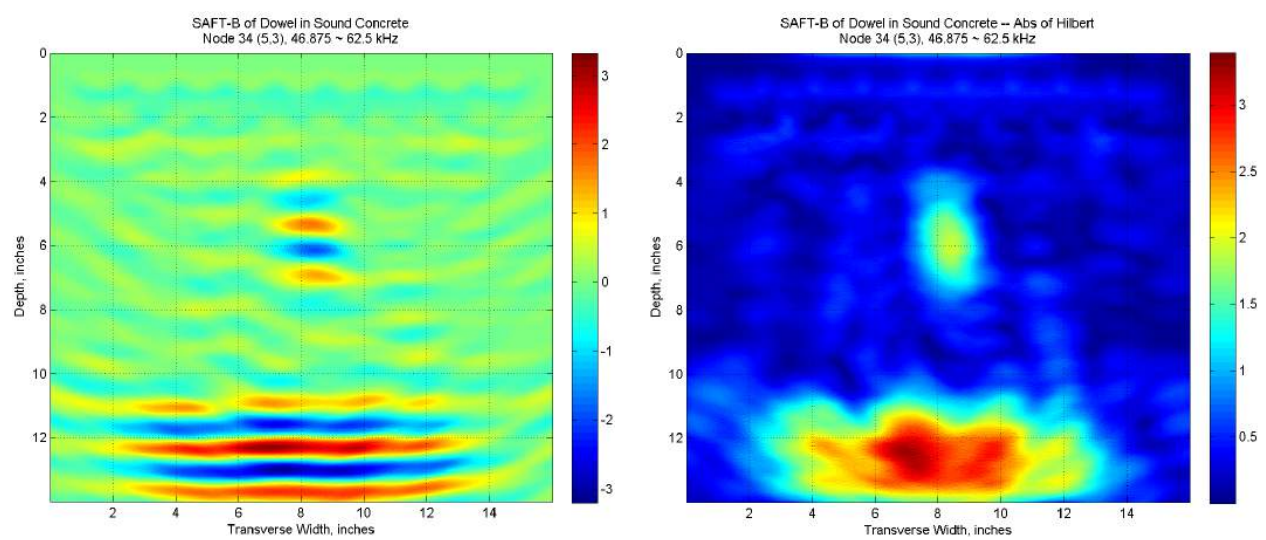

Fig. 15. Data details - data: Node 34; frequency range: $46.875 \sim 62.5 \mathrm{kHz}$; bandwidth: $15.625 \mathrm{kHz}$. 

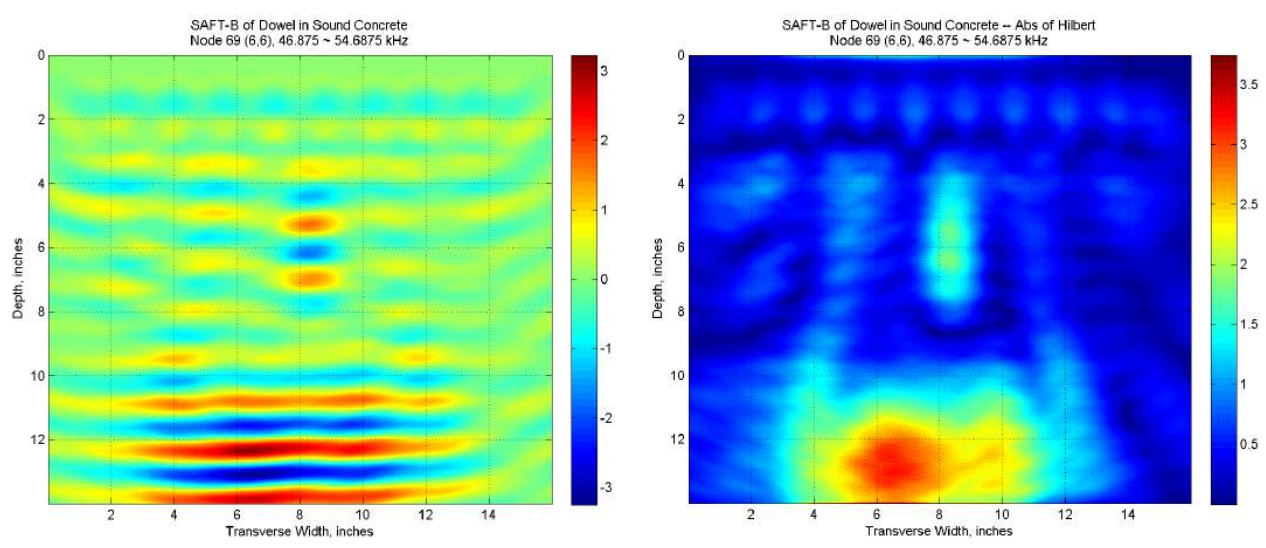

Fig. 16. Data details - data: Node 69; frequency range: $46.875 \sim 54.6875$; bandwidth: $7.8125 \mathrm{kHz}$.
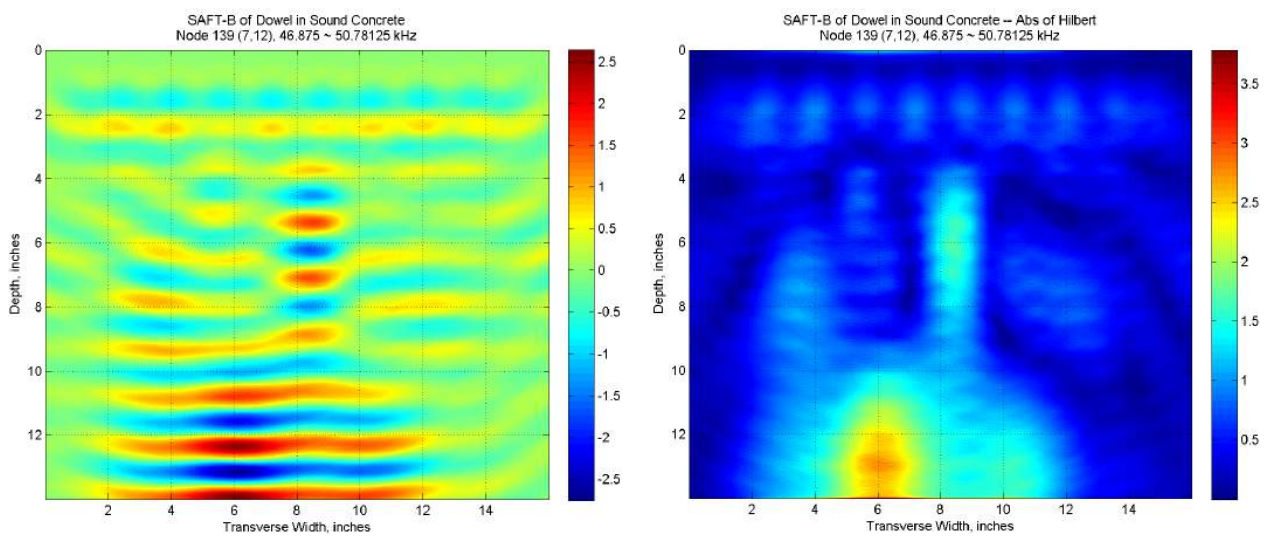

Fig. 17. Data details - data: Node 139; frequency range: $46.875 \sim 50.78125$; bandwidth: 3.90625 kHz.

\subsection{PROCEDURE OF THE FREQUENCY BANDING TECHNIQUE}

The wavelet decomposition with selective reconstruction is at its core a data pre-processing tool. Once a mother wavelet has been selected, the steps for generating the frequency-banded SAFT B-scans are as shown in Fig. 18. An initial examination of a larger selection of frequency bands is recommended in order to ascertain the "value" of the frequency bands of interest. The investigator may find that a particular frequency band contains too narrow a bandwidth and therefore little to no energy/information and should be discarded. Similarly, they may find that a particular frequency band appears very informative and should be decomposed further to allow for even more focused attention. All of these are possible using the wavelet packet decomposition plus reconstruction methodology proposed. An initial examination should be used to decide which frequency bands are worth continued extraction, further decomposition, or removal from the decomposition. In our case, we developed a procedure to process datasets in bulk. By eliminating uninformative decomposition levels, both computational time and data storage space were reduced. Each reconstructed time-series is used as the input to the SAFT algorithm and results in a SAFT B-scan image that specifically contains the energy from just the reconstructed frequency band. The computational intensive step of the procedure is not the wavelet decomposition and reconstruction but the SAFT algorithm. 
The benefit of "stitching" overlapping individual B-scans to form a single Panoramic B-scan greatly improves the readability and detection of defects and internal structures. However, it is not a required step of the procedure or necessarily always applicable, such as when a dataset contains a single capture.

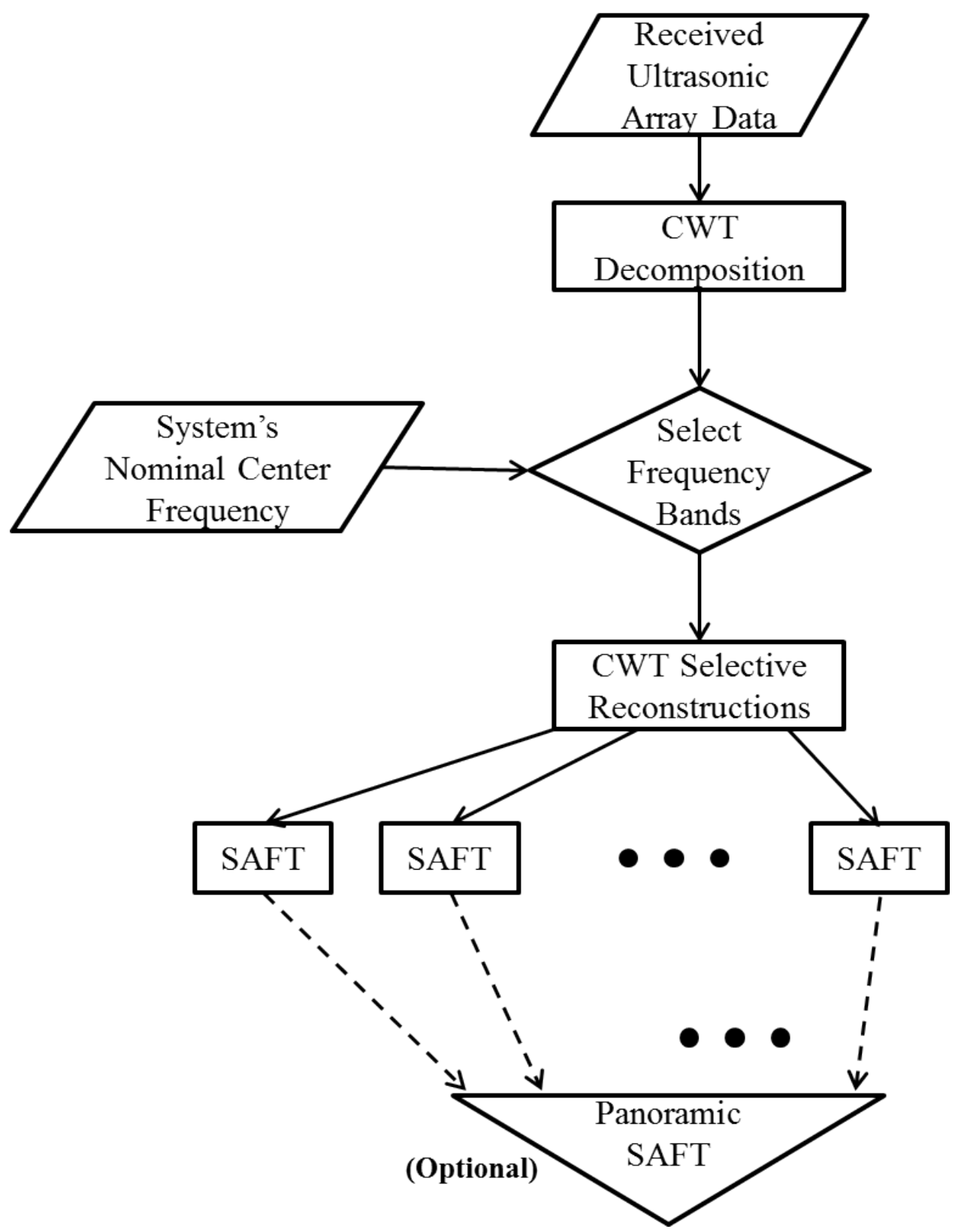

Fig. 18. Flowchart showing the typical work flow for frequency banding a dataset, generating the individual SAFT B-scans and then optionally constructing a panoramic SAFT B-scan from the individual SAFT B-scans. 


\subsection{PANORAMIC SAFT}

The idea and process of creating a Panoramic SAFT image is described in [6]. To achieve the most meaningful results, it was determined that the individual B-scan images should not be post-processed (i.e., taking the absolute value of the Hilbert transform of the SAFT results matrix) prior to the construction of the Panoramic SAFT.

If the absolute value of the Hilbert transform is taken first, as is typically done to SAFT results, all pixel values of the individual SAFT B-scans become positive. When the Panoramic SAFT image is created, it is impossible for the overlapping pixel regions to have any reduction in noise. The reduction of noise and boosting of legitimate reflections need the positive and negative values as directly returned by the SAFT algorithm. Otherwise, with only positive values, noise is only increased. However, the absolute value of the Hilbert transform of the Panoramic SAFT B-scan is taken, as it has benefits with respect to readability and content identification, over the "straight" SAFT results, panoramic or individual. 



\section{EXPERIMENT CONFIGURATION}

To determine the effects of the frequency banding technique for improving the detection, identification, and/or objective readability of SAFT reconstructions, the technique was applied to data that had previously been collected and analyzed using SAFT alone. Data previously collected as part of a test campaign that focused on evaluating the performance of several different concrete inspection methods was used [6]. As the frequency banding technique is transparent to the imaging algorithm and can be applied to any time-series data, the data collected during the test campaign provides a ready source of data, previous SAFT results, and ground truth for comparison.

\subsection{EQUIPMENT}

The data was collected using a MIRA version 1 system (Fig. 19). The MIRA is a commercially available ultrasonic linear array inspection system manufactured by Acoustic Control Systems. The version of the system used to collect the data to which the frequency banding technique was applied consists of an array of 40 dry point contacts arranged in four rows of ten each. The system has a nominal center frequency of $50 \mathrm{kHz}$ and samples at one mega-sample per second (1Ms/sec). Each column of four transducers is treated as a unit for a total of 10 unique channels. The lowest level data available from the MIRA version 1 are these "merged" channels. For more details on the linear operation and acquisition scheme of the MIRA version 1, see [1] and [7].

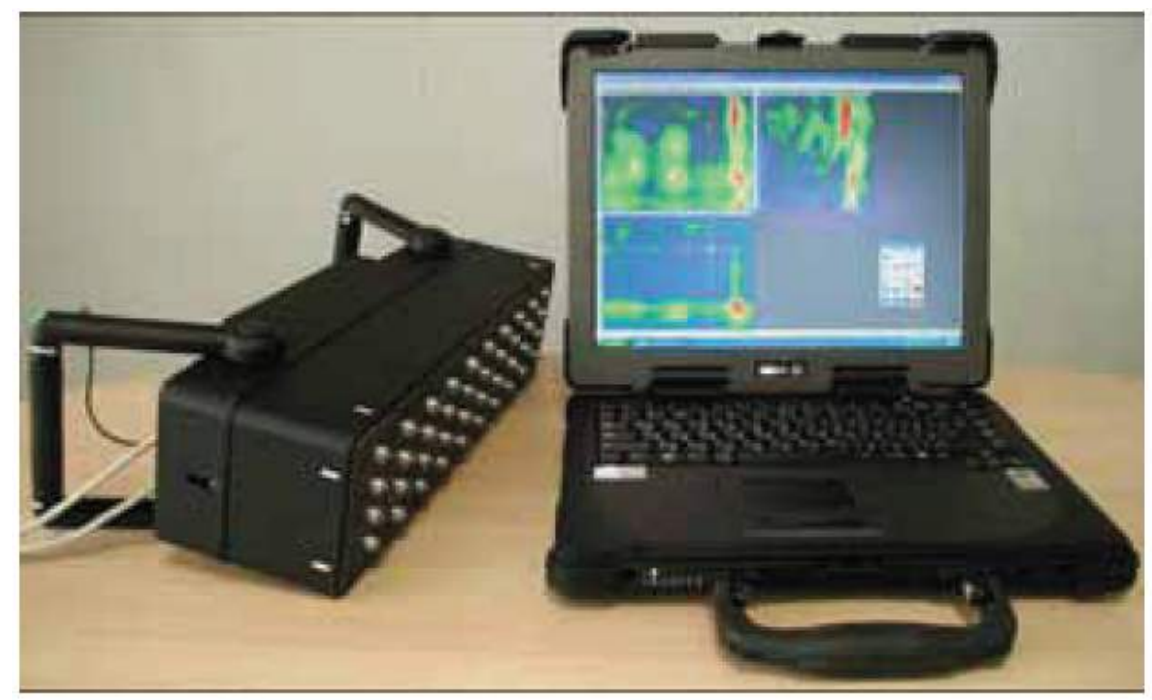

Fig. 19. Acoustic Control Systems MIRA version 1 [7].

\subsection{TEST SPECIMENS AND DATA COLLECTION}

Data was collected using the MIRA system version 1. To begin the development of the frequency banding technique, data was collected from two specimens of known sound concrete. This data was used in the selection of the mother wavelet and as a simple highly controlled reconstruction target. This was possible because one specimen was a 12 in. thick sound concrete specimen containing no defects or internal structures of any sort. The second specimen was a 12 in. thick sound concrete specimen containing a single $1.5 \mathrm{in}$. diameter steel dowel rod centered at mid-depth. The development of the frequency banding technique using the $1.5 \mathrm{in}$. dowel rod specimen will be discussed first.

Once the technique was developed and refined using the sound concrete plus dowel rod specimen, the data collected from two test specimens each measuring 80 in. $\times 57$ in. $\times 10$ in. housed at the Florida 
Department of Transportation's Nondestructive Evaluation (NDE) Validation Facility in Gainesville, Florida was analyzed [8]. These two test specimens were specially designed and fabricated to provide test cases and ground truth for known NDE problem areas.

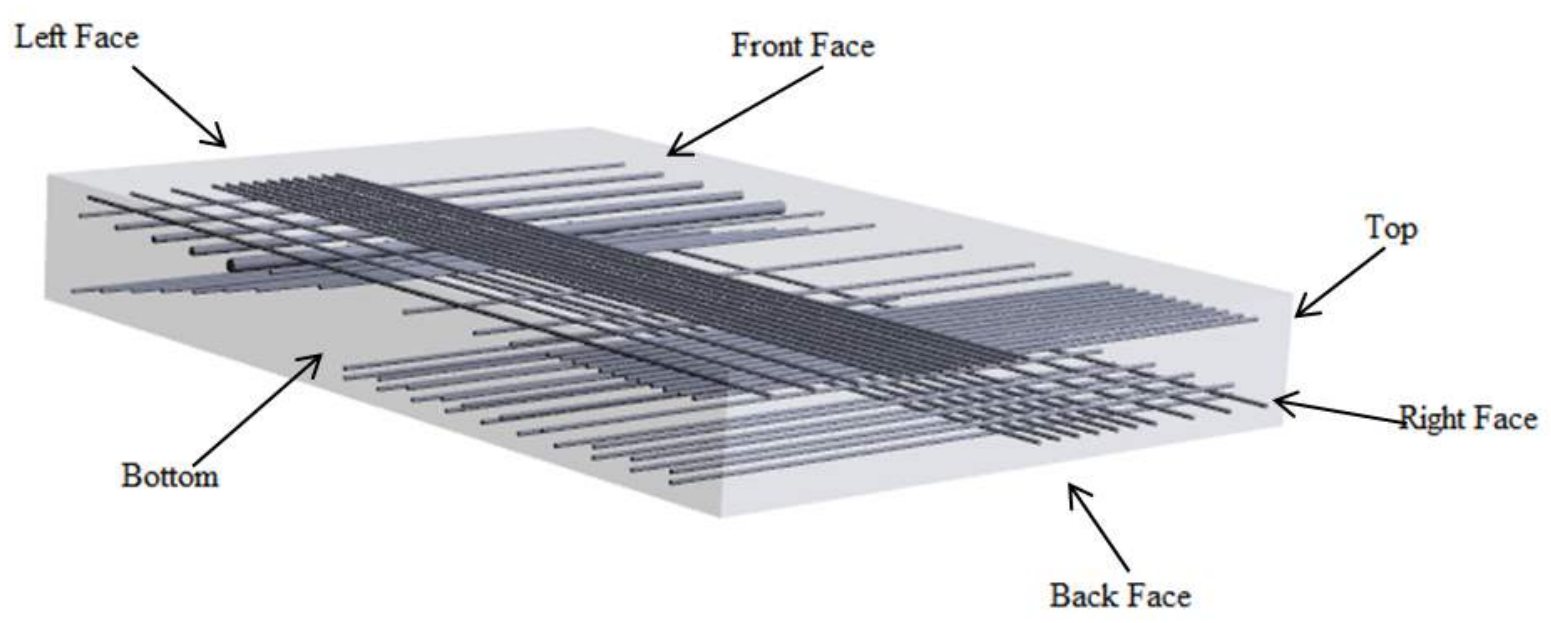

Fig. 20. Orientation and location of the rebar mats in the rebar test specimen (Specimen-2).

The first specimen, known as Specimen-2, contains a variety of rebar mat structures consisting of various diameters and orientations with horizontal, vertical, and depth spacing, as shown in Fig. 20. The second specimen, known as Specimen-6, contains a variety of analogs representing surface breaking cracks, voided rebar/bonding voids along rebar, and honeycombing. A schematic of Specimen- 6 and its contents is shown in Fig. 21.

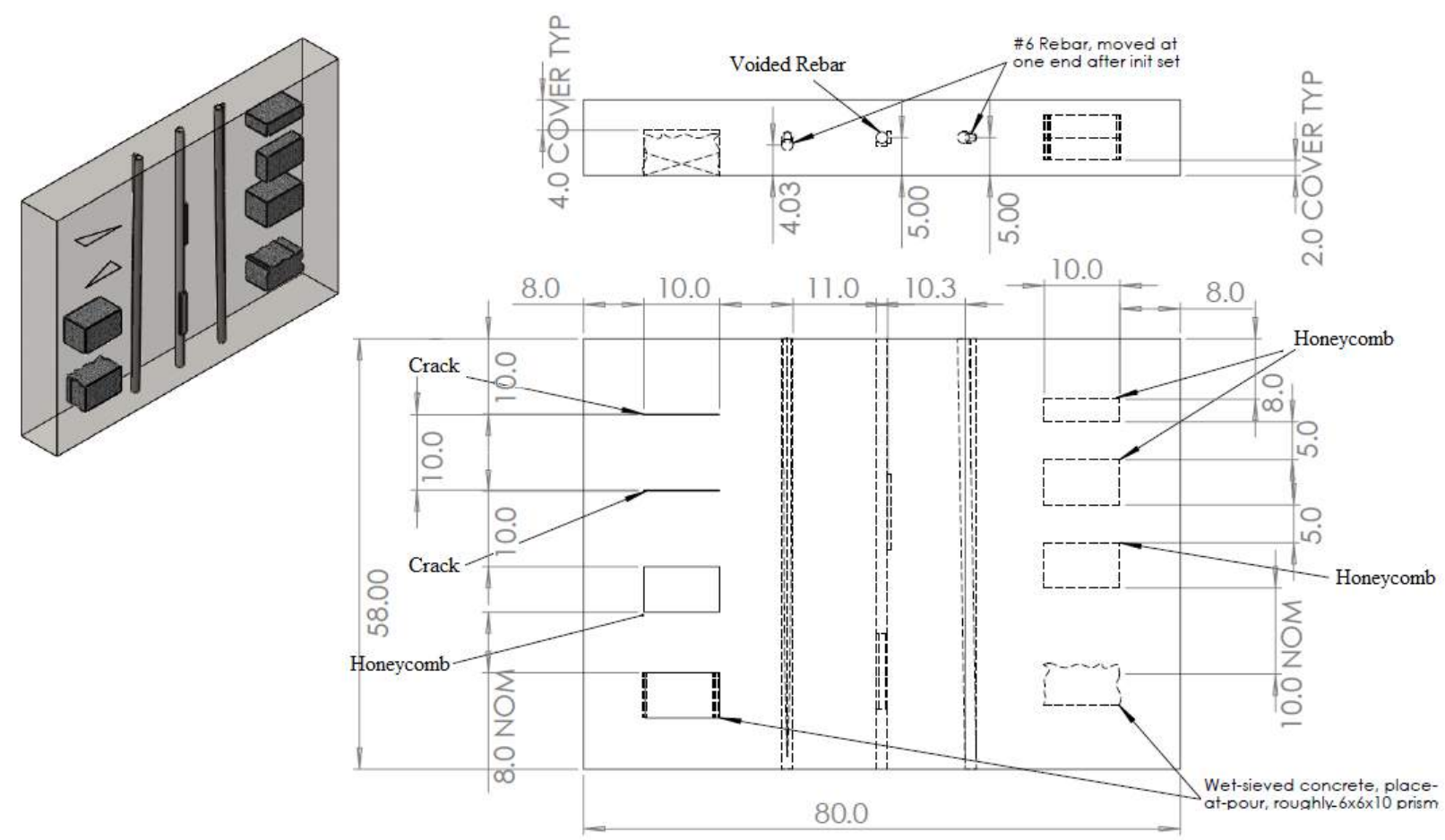

Fig. 21. Schematic of the honeycomb, crack, and rebar bonding defects contained in Specimen-6. 


\subsection{COLLECTION PROCEDURE}

Both horizontal and vertical scans were collected using the MIRA version 1 system. For horizontal scans, the long axis of the device was oriented horizontally, with the center of the array $8 \mathrm{in}$. from the left edge of the specimen (Fig. 19). A capture was collected along the horizontal scan line in 4 in. steps and stopped $8 \mathrm{in}$. from the right edge. The first horizontal scan was collected at the bottom edge of the specimen with the MIRA flush to the bottom. A horizontal scan set was taken in 4 in. vertical steps [1]. Figure 22 shows the location of each horizontal set and the positions that make up each of those sets.

For the vertical scans, the long axis of the MIRA unit was oriented perpendicular to the long axis of the specimen. The positions were collected by moving the unit in 4 in. steps from bottom to top, starting with the center of the MIRA array 8 in. from the bottom edge of the block and stopping when the center of the array was $8 \mathrm{in}$. from the top edge. Vertical scan sets were collected in $4 \mathrm{in}$. horizontal increments starting at the right edge and progressing left across the scanned surface [1]. Figure 23 shows the location of each vertical set and the positions that make up each of those sets.

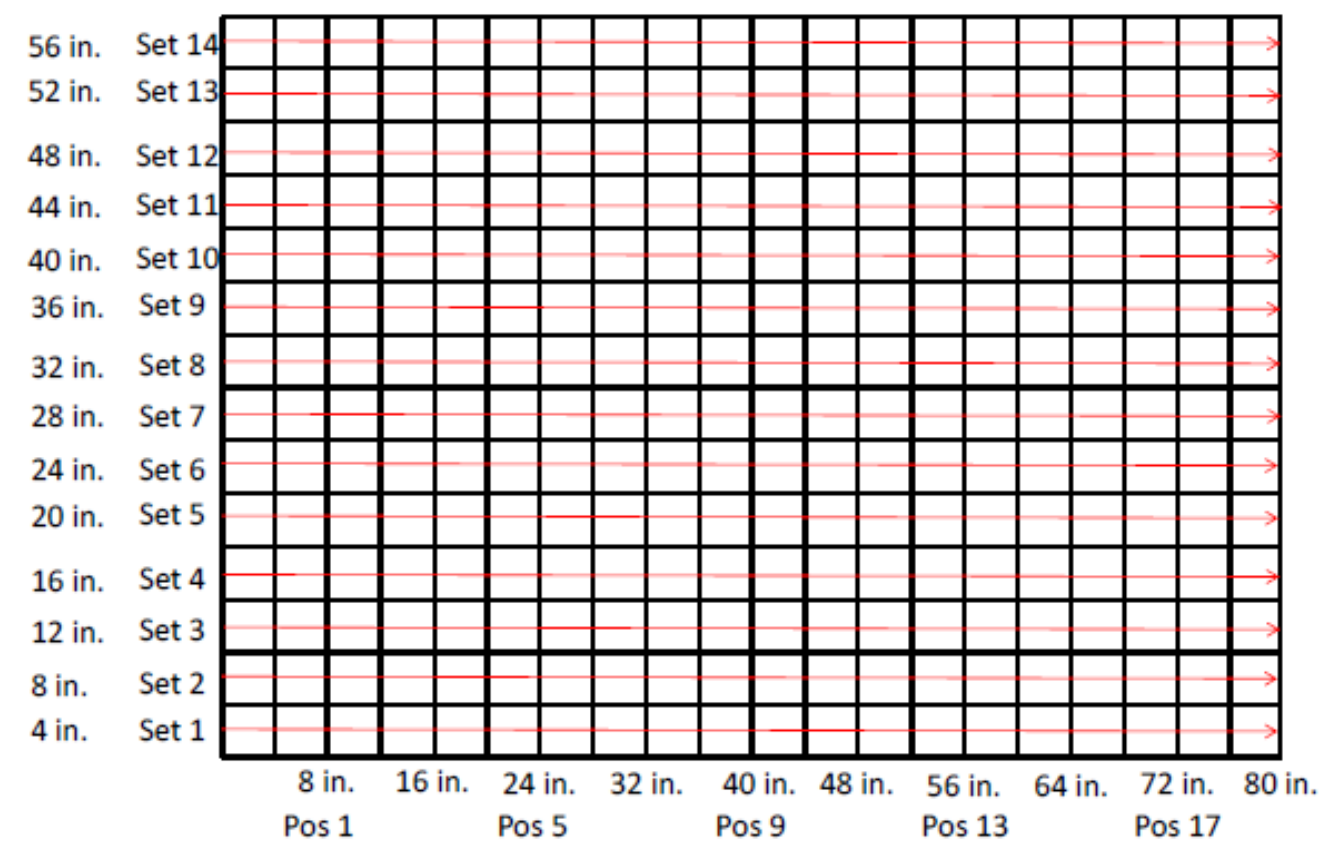

Fig. 22. Locations of each horizontal scan set and its positions. The red lines with arrowheads are the scan sets. 


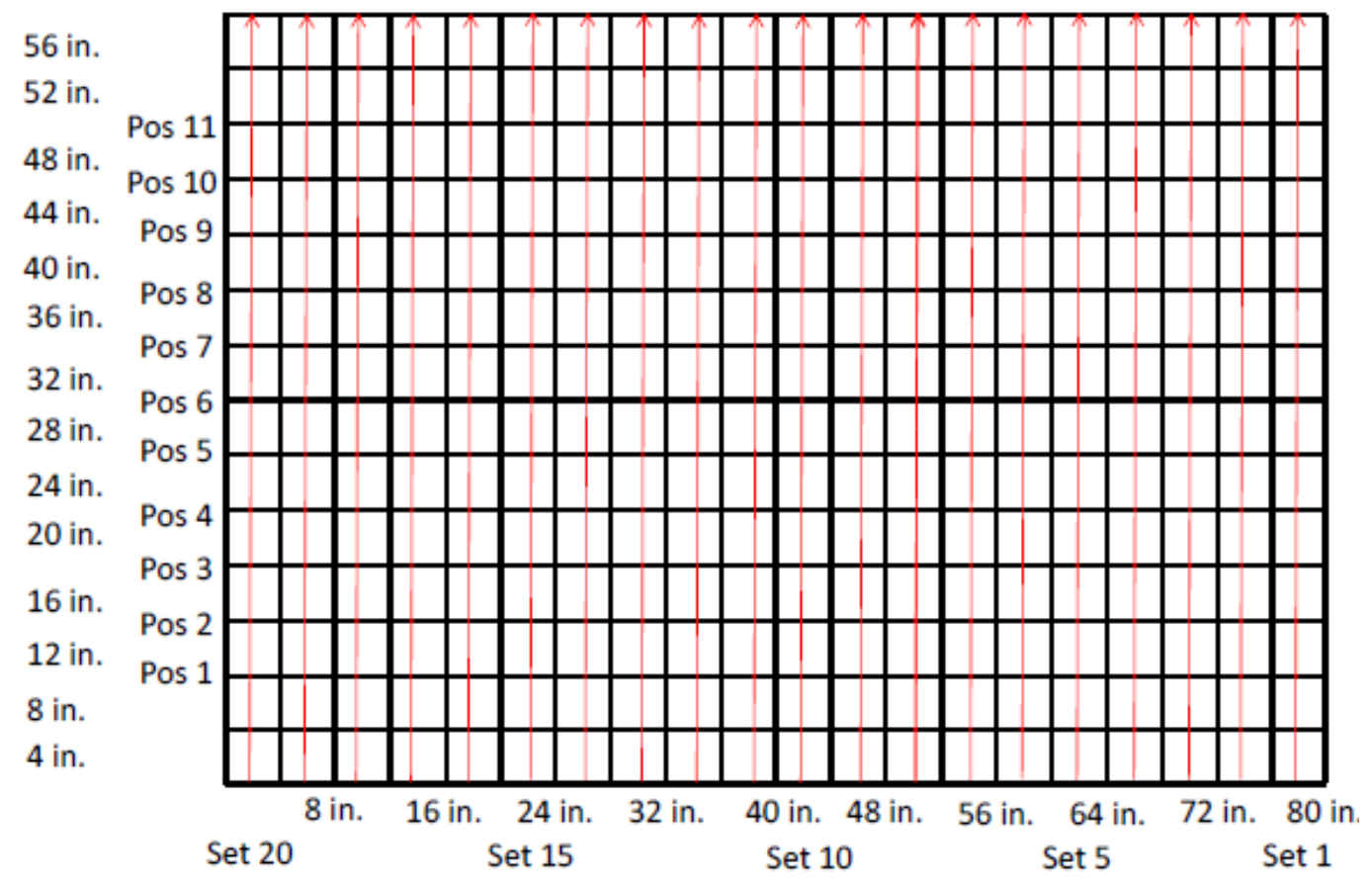

Fig. 23. The locations of each vertical scan set and its positions [1]. 


\section{RESULTS}

\subsection{COMPLEX FLORIDA NDE SPECIMENS}

After examining various frequency bands, narrowing the frequency content presented as input to the SAFT algorithm was found to aid in distinguishing defects and internal structures above the background noise.

In Fig. 24, a crack oriented parallel to the scan path and whose closest point to the surface scanned has $7 \mathrm{in}$. of cover is shown. The scan passed directly over a 1/16 in. crack, which was surface breaking on the opposite side of the specimen from the scan path. The scan also grazed a honeycomb analog shown on the right-hand side of Fig. 24(a-c). Figure 24(b) shows the SAFT results without any frequency banding. Figure 24 (c) shows the SAFT results with frequency banding. The almost complete lack of any back wall reflection for the full length of the crack is unique to the frequency-banded result in Fig. 24(c). Also, the location of the rightmost rebar more closely matches the design drawings, as shown in Fig. 24(a). It is also worth pointing out that the result in Fig. 24(c) is from the frequency band containing the second harmonic of the nominal center frequency. Effectively, second harmonic imaging was performed without the need for any additional processing, equipment, or otherwise by using the frequency banding technique as a pre-processing step applied to the data.

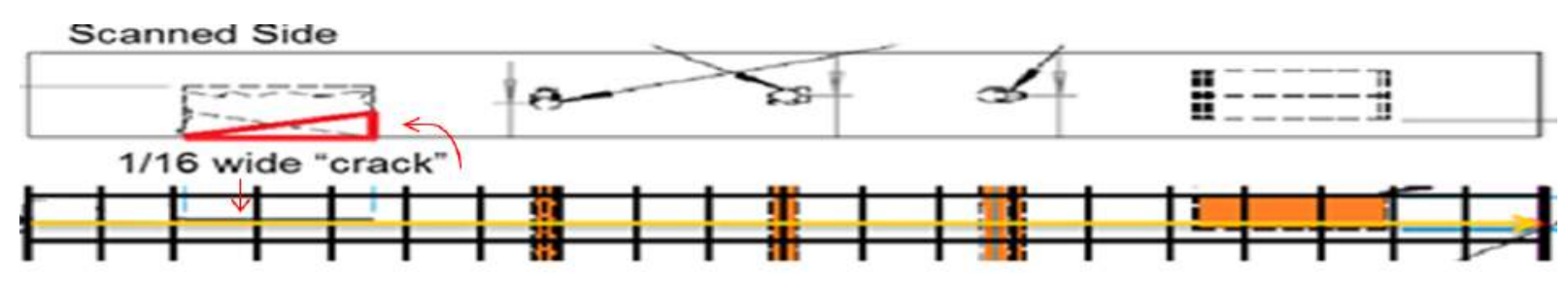

(a)

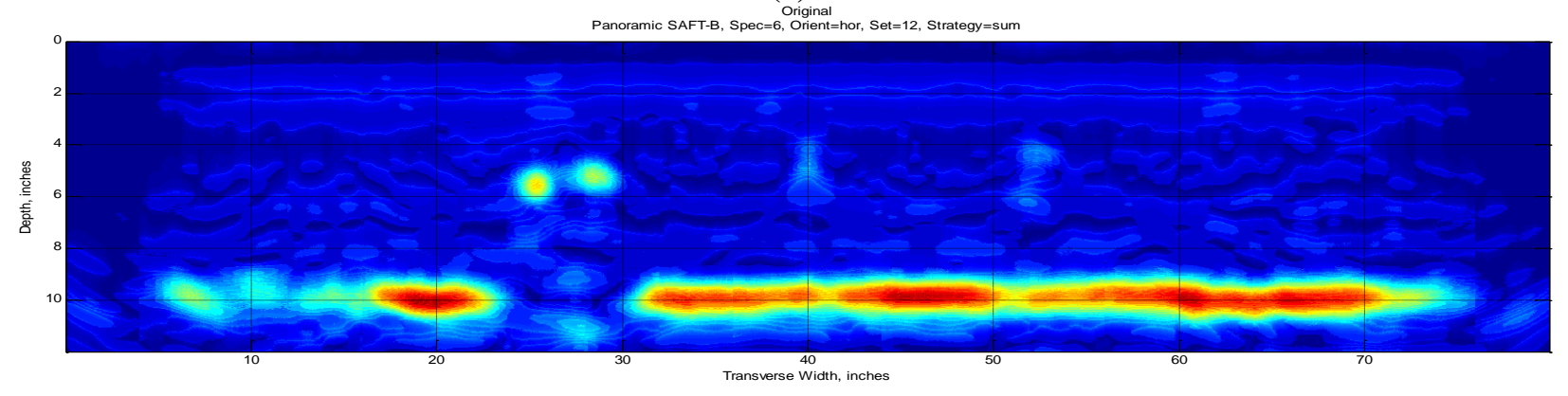

(b)

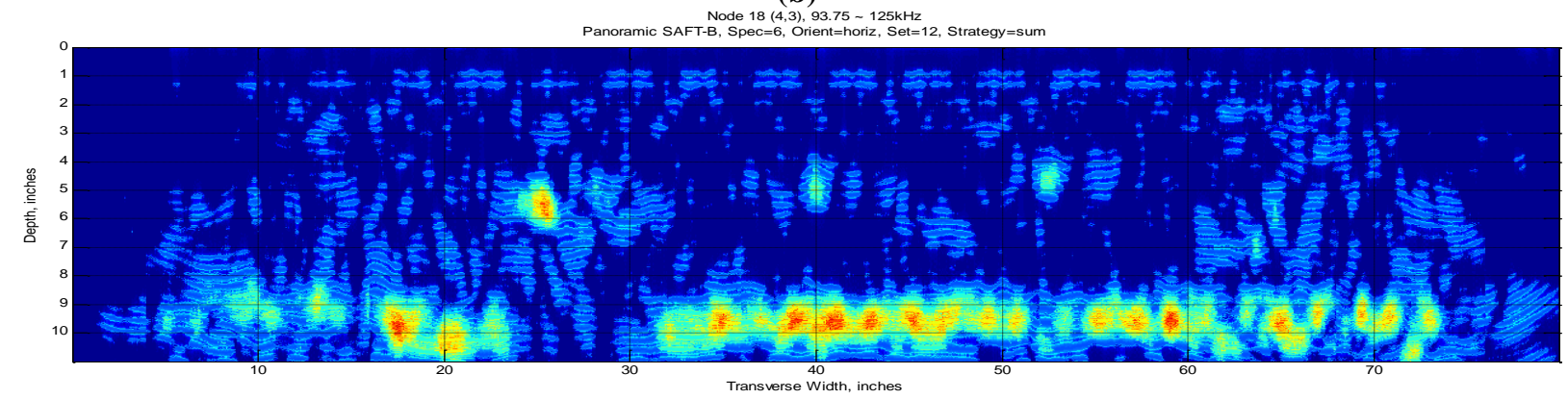

(c)

Fig. 24. (a) Ground truth for Specimen-6, horizontal set 12, (b) the Panoramic SAFT reconstruction using the dataset as is, and (c) the results of using the frequency band containing the second harmonic of the nominal center frequency. 
The results for honeycomb detection show improvement as well. Figure 25 shows a vertical scan that passed over four honeycomb analogs/prisms. The areas of honeycomb are identifiable by the significant shadowing of the back wall reflection they cause. Three of the honeycomb prisms were pre-cast, placed in the form, with the concrete mix carefully packed around them as it was poured. A fourth honeycomb prism was created by removing a portion of the mix after it had been poured and was then sieved back into the opening from which it was removed [1]. The frequency band containing the second harmonic of the MIRA v1's nominal center frequency showed remarkably clear indications of honeycomb due to back wall shadowing. The leftmost and rightmost edges of both Fig. 25 (a) and Fig. 25 (c) suffer from the fact that there were not any additional MIRA steps at the upper and lower edges of the specimen. Because there is only one acquisition in these locations, there was simply no data to merge as part of the panoramic process. All other positions along the scan benefited from 4 in. of overlap from the neighboring collection positions of the scan.

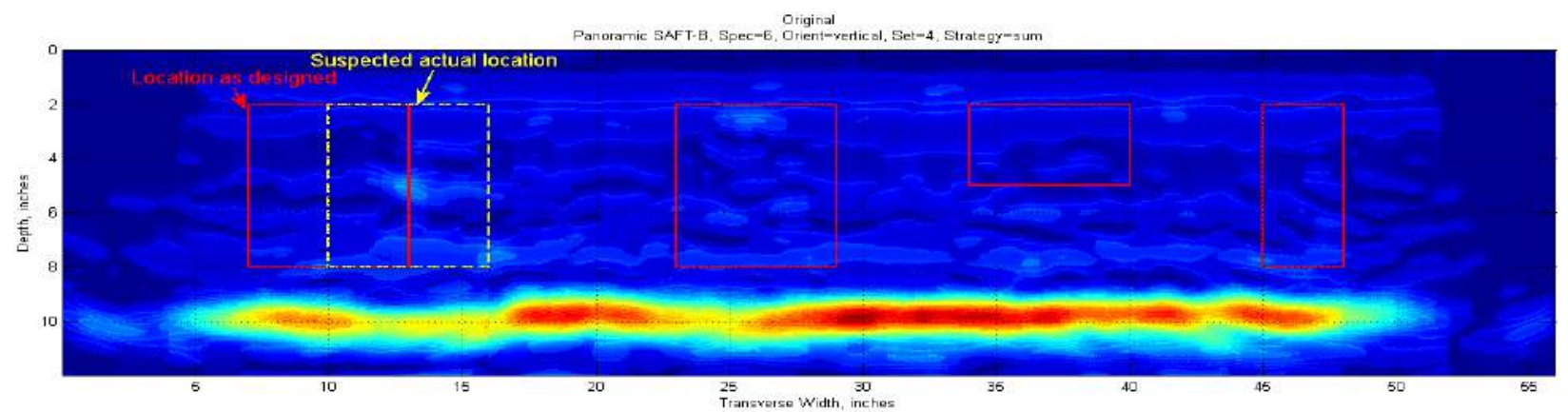

(a)
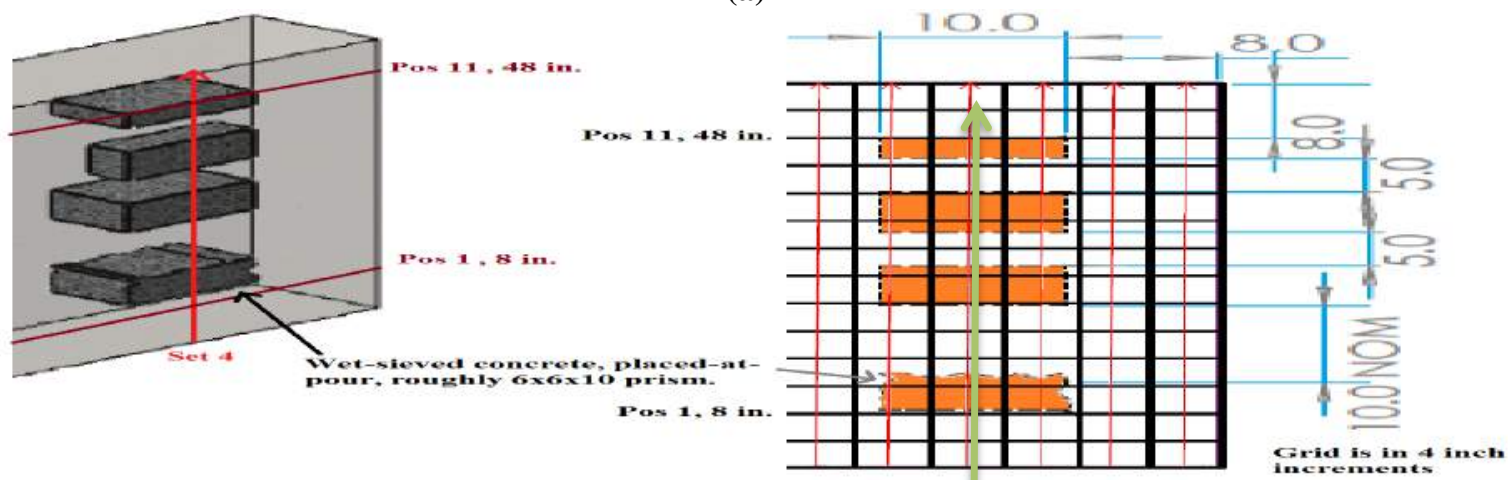

(b)

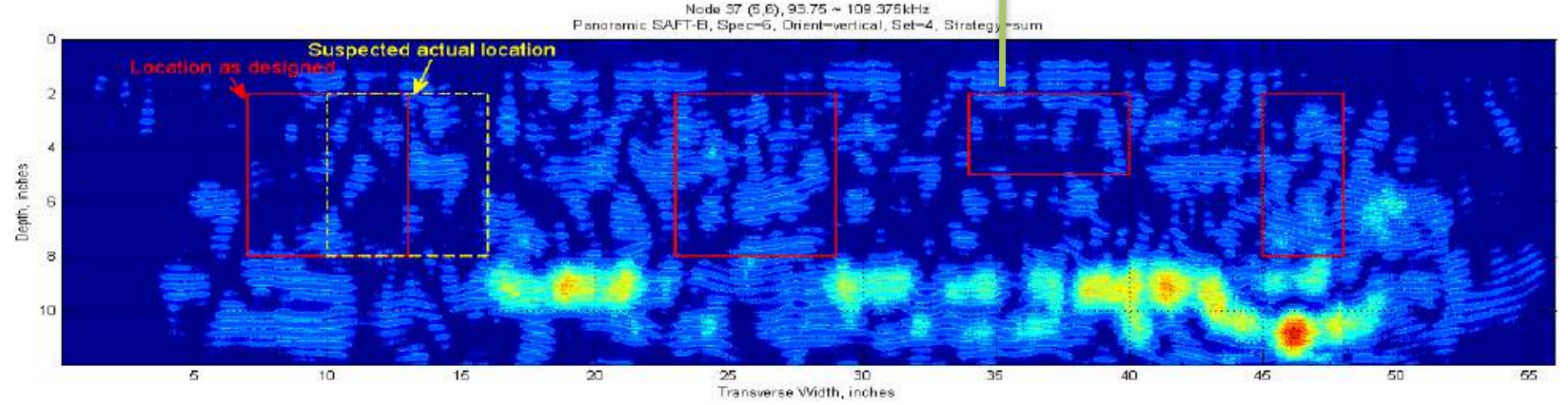

(c)

Fig. 25. The Panoramic SAFT B-scans of a vertical scan of Specimen-6, which passed over four different honeycomb prisms/analogs. (a) original Panoramic SAFT image, (b) ground truth showing the locations of the honeycomb prisms as designed, and (c) Frequency-banded Panoramic SAFT. 
The prism that was placed-at-pour was placed after the entire specimen was poured. A portion of the pour was shoveled out and replaced with a wet sieved mix by hand. As this was a very manual process and involves wet concrete, it is believed that this prism's location is slightly shifted from the design drawings.

The benefits of the technique are also quite clear, as seen when comparing the original Panoramic SAFT results, in Fig. 26(a), to the results obtained when the original $500 \mathrm{kHz}$ bandwidth is segmented to a roughly $30 \mathrm{kHz}$ bandwidth containing the systems nominal center frequency, as shown in Fig. 26(c). The strong peaking pattern at the lower right-hand side of the results, shown in Fig. 26(c), is the result of where the application of the Hilbert transform has "enveloped" the rebar indications into the back wall reflection due to their closeness, as shown in Fig. 26(b).

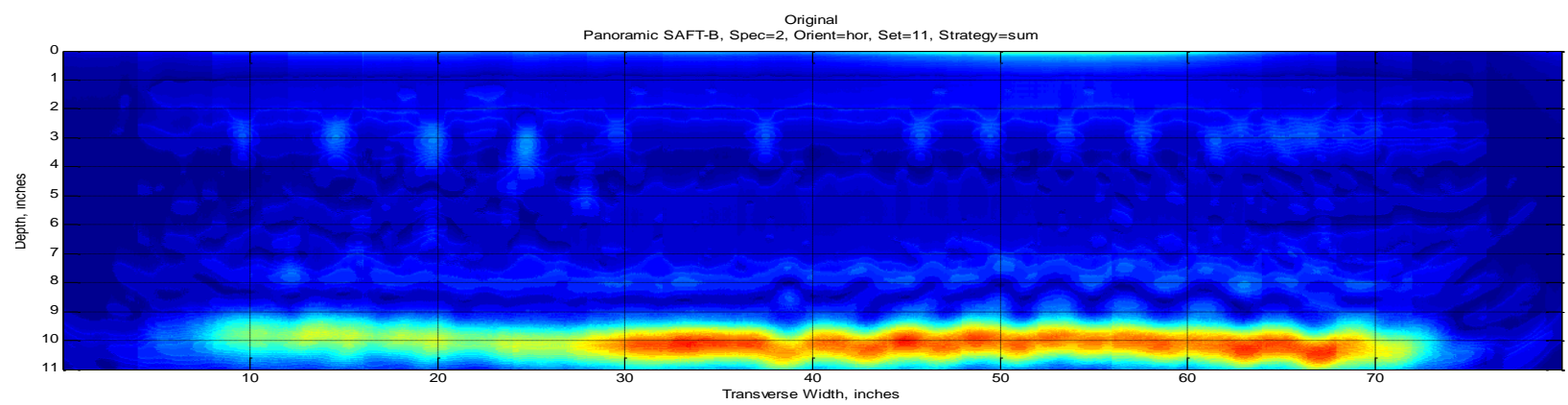

(a)

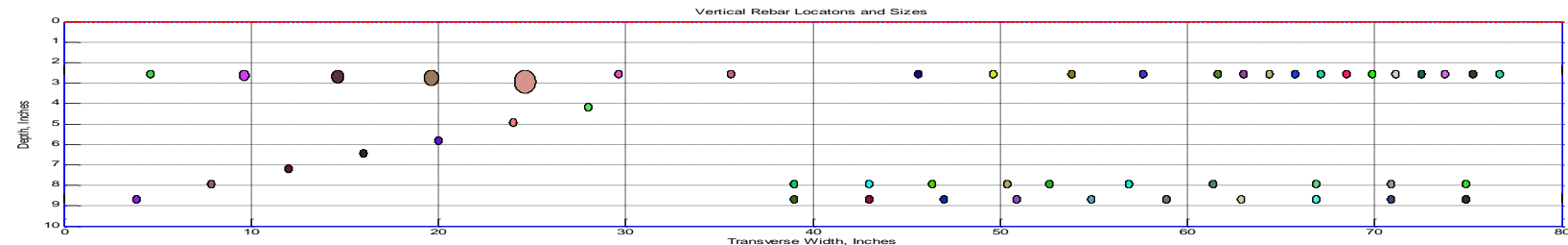

(b)

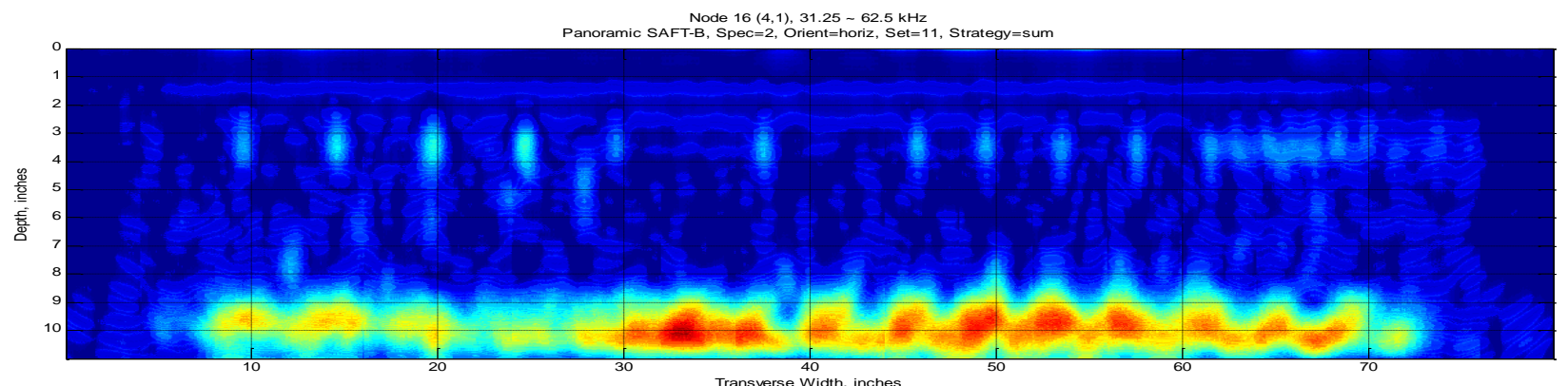

(c)

Fig. 26. Comparison between Panoramic SAFT B-scans with and without frequency banding. (a) Panoramic SAFT B-scan generated from a horizontal scan set 11 of Specimen-2 using the data as is, (b) schematic representation of the rebar perpendicular to the scan as designed, (c) Panoramic SAFT B-scan using an approximately $30 \mathrm{kHz}$ wide bandwidth containing the $50 \mathrm{kHz}$ nominal center frequency. 



\section{SUMMARY OF THE FREQUENCY BANDING TECHNIQUE}

As has just been shown, segmenting ultrasonic data into frequency bands to which SAFT, or any other imaging algorithm that works on time-series data, can provide several advantages. It provides improvements in the background noise and spurious artifacts in SAFT reconstructions. It allows harmonic imaging to be performed without any equipment or imaging algorithm modifications. It improves the readability of the SAFT results and is a step towards the more objective identification of internal structures and defects in concrete structures.

Traditional filters could be used to achieve the same frequency segmentation effects of the wavelet packet decomposition, but the flexibility of determining which frequency bands are beneficial from the data would be limited as parametric choices such as the filters, their stop and pass bands, the frequency leakage, the ripple, and the computation time, to name a few, would need to be calculated and adjusted for every situation. The wavelet approach with its properties tied to the mother wavelet requires less preanalysis and a priori knowledge of the inspection system and the structure under test.

Overall, one of the greatest advantages to this technique is its transparency with respect to the acquisition system and the image reconstruction algorithm. It provides marked improvements in the readability and identification of rebar by improving the contrast and improves the detection of low-level defects identified by reduction and/or the absences of back wall reflections.

It is clear that an accurate propagation velocity is needed to provide accurate localization of objects (e.g., honeycomb, rebar). Ideally, a method for calculating the average propagation velocity is preferred as the elasticity, water content, and other factors change as concrete ages and its environment changes. However, these empirically calculated velocities can contain significant error if the data used in the measurement was close to a face of the concrete specimen. This brings up another aspect in need of consideration. When a large concrete structure is made of multiple pours of concrete done over several days or even weeks, the junction of neighboring pours may cause signal boundary losses. It is believed that such boundary losses will be evident when analyzing the data.

\subsection{SECOND HARMONIC IMAGING}

As part of our investigation and experiments with decomposing ultrasonic data into distinct frequency banding, we noticed that the frequency band(s) containing the second harmonic of the nominal center frequency displayed a consistent, repeatable detection of rebar. In fact, it displayed many of the desired characteristics, including greatly enhanced object identification of the rebar elements. This effect has previously been identified in the field of medical ultrasound. The use of the second harmonic for imaging is now one of primary, if not the primary, imaging modes used in medical ultrasound [9].

The discovery of the advantages of second harmonic imaging in medical ultrasound resulted from the realization that tissue, while close to a linear medium, is a non-linear medium [9]. Concrete is a very nonlinear medium. It is therefore no surprise that the benefits seen in medical ultrasound are directly applicable to ultrasonic inspection of concrete. In fact, the Panoramic SAFT images shown in Fig. 24(c) and Fig. 25(c) were both generated using the frequency band containing the second harmonic of the MIRA's nominal center frequency. The adoption of second harmonic imaging can be achieved using the frequency banding technique described in detail earlier. The use of additional harmonics is possible as well but will likely necessitate an increase in the power of the excitation pulses in order to create higher order harmonics with enough signal strength for their reflections to be received. Additionally, the bandwidth of the transceivers must be considered as frequencies above the fundamental are considered. 



\section{TOTAL FOCUSING METHOD - IMPLEMENTATION OF THE TOTAL FOCUSING METHOD FOR MIRA VERSION 1}

\subsection{THE ALGORITHM}

The Total Focusing Method (TFM) was originally developed to reconstruct ultrasonic signals from metals [10]. The method shares many similarities to other traditional delay and sum algorithms, such as the SAFT [11]. Equation 4 shows the reconstruction model for a SAFT approach, while Eq. (5) shows the reconstruction model for the TFM. The variables are defined as follows: $p_{k}$ is the intensity of pixel $k$, $S_{i, j}(t)$ is the amplitude at time $t$ of the acoustic signal transmitted and received by transducers $i$ and $j$, respectively, $\mathrm{N}$ is the number of transducers, $d_{m, n}$ is the Euclidian distance from element $m$ to element $n$, and $v_{c}$ and $v_{m}$ are the acoustic speeds of the couplant and the media to reconstruct, respectively. Finally, elements $a$ and $b$ are exclusive to the TFM algorithm and represent coordinate points on the surface of the media, as shown in Fig 27. Note that the main difference between SAFT and TFM is how the methods time delay the measured ultrasonic signals, $S_{i, j}(t)$. In SAFT, there are three assumptions: the transducers are in complete contact with the surface of the media, the media acoustic speed $v_{m}$ is constant, and $v_{c}=v_{m}$. Therefore, signal delay is controlled by a single time parameter, $t=\left(d_{i, k}+d_{k, j}\right) / v_{m}$. For real world scenarios, it is likely that the transducers are not completely in contact with the media surface and the acoustic velocity varies throughout the media. This is particularly true when imaging concrete. TFM provides a better imaging model by accounting for separation between the transducers and the media surface and the change in acoustic speed at the couplant/media interface.

$$
\begin{gathered}
p_{k}=\sum_{i=1}^{N} \sum_{j=1}^{N} S_{i, j}\left(\frac{d_{i, k}+d_{k, j}}{v_{m}}\right) . \\
p_{k}=\sum_{i=1}^{N} \sum_{j=1}^{N} S_{i, j}\left(\frac{d_{i, a}+d_{b, j}}{v_{c}}+\frac{d_{a, k}+d_{k, b}}{v_{m}}\right) . \\
a=\operatorname{argmin}_{a} \frac{d_{i, a}}{v_{c}}+\frac{d_{a, k}}{v_{m}} . \\
b=\operatorname{argmin}_{b} \frac{d_{b, j}}{v_{c}}+\frac{d_{k, b}}{v_{m}} .
\end{gathered}
$$

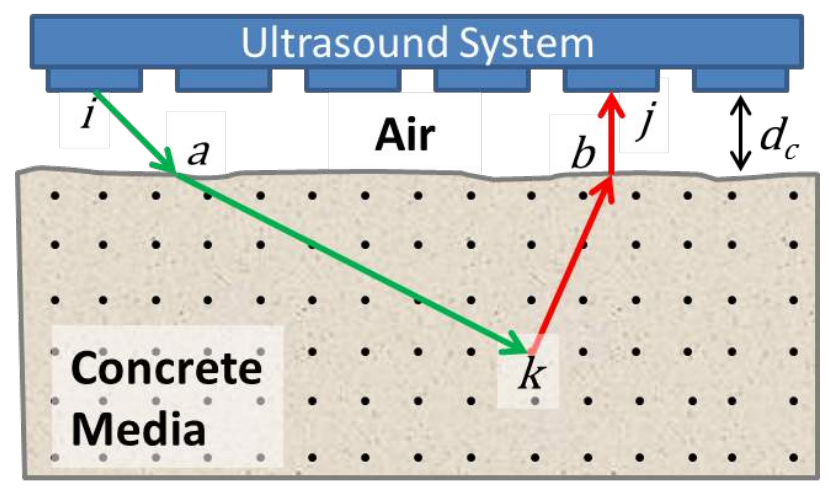

Fig. 27. Illustration of acoustic wave tracing for TFM.

As shown in Fig. 27 for TFM, there is a couplant gap $d_{c}$ between the transducers and the media surface. The couplant could be air, ceramic, water, an acoustic gel, or a combination of these.

Additionally, the ray connecting the transducer $i$ to the reconstruction pixel $k$ may not be a straight line due to the differences in acoustic speeds $v_{c}$ and $v_{m}$. 
The deviation point or refraction point $a$ can be computed following the principle of least time, also known as Fermat's Principle. The principle states that the wave will traverse the path that takes the least time. Consider the path from the transmitter $i$ to the pixel $k$. If $v_{c}=v_{m}$, then a straight path between $i$ and $k$ has the shortest traverse time. However, if $v_{c} \neq v_{m}$, the refraction point $a$ is given by Eq. (5.a), which searches for the coordinate $a$ that minimizes the travel time through elements $i, a$, and $k$. The same procedure applies to the shortest travel time for waves reflecting at pixel $k$, intersecting $b$, and arriving at receiver $j$. Note that this optimization approach is performed for all combinations of transmitters, receivers, and reconstructions pixels. The required optimization step makes TFM highly computationally expensive relative to SAFT. For optimal results, the couplant and the gap parameter $d_{c}$ are known as a priori. For the results below, the couplant was assumed to be air $\left(v_{c}=349.361 \mathrm{~m} / \mathrm{s}\right)$ and $d_{c}$ was set to a value between $4.5 \mathrm{~mm}$ and $6.5 \mathrm{~mm}$.

\subsection{THE IMPLEMENTATION}

The TFM implementation has three main sections: signal smoothing, Full Matrix Capture (FMC) calculation, and reconstruction. In the first step, the acoustic signals are filtered with a Butterworth digital low-pass filter with a cut-off frequency selected empirically from the data to reconstruct and typically in the $\mathrm{kHz}$ range. The FMC calculation consists of a reorganization of the acoustic signals in a threedimensional matrix - the matrix rows and columns correspond to transmitter and receiver indexes, respectively, and the third dimension corresponds to the time domain. This format is preferred in order to vectorize some of the reconstruction processes. The final step is the reconstruction of the concrete media, which is formally described in Eq. (6). Note that the original TFM equation was adapted to the MIRA version 1 system, which only receives with the transducers at the left of the transmitting transducer.

$$
\begin{gathered}
p_{k}=\sum_{i=1}^{M} \sum_{j=i+1}^{N} \alpha_{i k j} \omega_{i k j} S_{i, j}\left(t_{i, k, j}\right) . \\
\alpha_{i k j}=e^{\alpha_{o} d_{i, k, j}} . \\
\omega_{i k j}=\omega_{i, a} \omega_{j, b} . \\
w_{m, n}=\left(\left(4-2 \sin ^{2} \theta_{m, n}\right) \cos \theta_{m, n}\right) / F_{o}\left(\sin \theta_{m, n}\right) . \\
F_{o}(x)=\left(2 x^{2}-4\right)^{2}-4 x^{2} \sqrt{x^{2}-1} \sqrt{x^{2}-4} .
\end{gathered}
$$

The reconstruction process starts by defining the reconstruction space. The depth of the reconstruction image and the transducers' width and pitch are given as a priori. The reconstruction image width is set to the width of the ultrasonic array (i.e., number of transducer elements times the pitch between elements), and the pixel size is set to half the width of a transducer element. We compute the refraction points $a_{i, k, j}$ and $b_{i, k, j}$, for each pixel $k$ and transducer pair $\langle i, j\rangle$, with a nonlinear bounded hybrid optimization function (i.e., combination of successive parabolic interpolation and golden section search). After calculating the refraction points, we can then compute the delay time $t_{i, k, j}$ and the weights $\alpha_{i k j}$ and $\omega_{i k j}$ for attenuation and source/sensing divergence correction, respectively. The attenuation correction weight amplifies reflections with longer travel paths in order to compensate for scattering and absorption effects. Linear and non-linear attenuation correction methods were implemented. For the linear method, the weight $\alpha_{i k j}=t_{i k j} / t_{\max }$, where $t_{i k j}$ is the travel time from transmitter $i$, to pixel $k$, and back to receiver $j$ and $t_{\max }$ is the longest possible travel time for any path. The non-linear method is computed with Eq. (7), where the variable $\alpha_{o}$ is the attenuation coefficient for the media in Nepers per meter and $d_{i, k, j}$ is the traveled distance. 
The attenuation coefficient for concrete imaged with a central frequency of $50 \mathrm{kHz}$ is between 1.75 and 3.25 Nepers per meter (assuming attenuations varies linearly with frequency). Source and sensing divergence correction rectifies the non-idealities of transmitters and receivers, respectively. In general, the amplitude of the emitted acoustic varies across the field of view of the transducer. In the same wave, the sensitivity of the receivers varies with the wave incidence angle.

The weight $\omega_{i k j}$ accounts for such non-idealities. In Eq. (8.b), $\omega_{m, n}$ is the profile model for transmitters and receivers. As illustrated in Fig. 28(a), $\theta_{m, n}$ is the divergence angle between a vector normal to the transmitter/receiver $m$ and a vector passing through the center of the transmitter/receiver and the corresponding refraction point, $n$. As shown in Fig. 28(b), $\omega_{i k j}$ is bell-shaped, where rays that diverge from the normal are penalized with a lower weight.
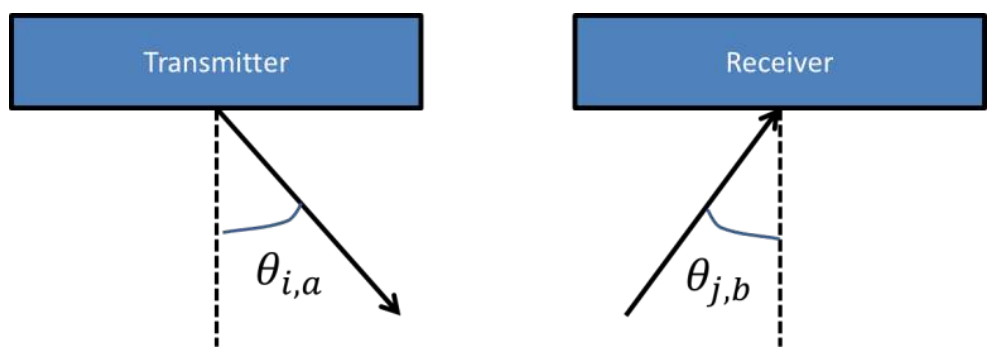

(a)

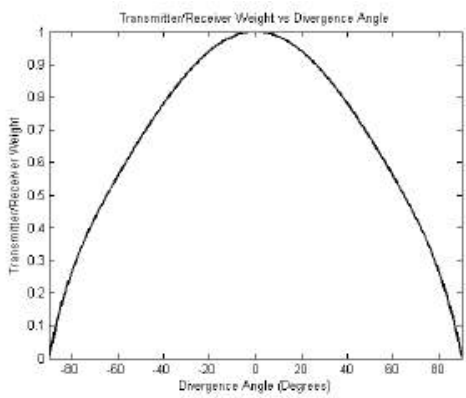

(b)

Fig. 28. (a) Illustration of divergence angle for transmitters and receivers and (b) plot of weight values as a function of divergence angle.

\subsection{RESULTS}

In this section the performance of TFM is measured relative to SAFT. Since the MIRA v1 system was rented and not purchased, we do not know the exact system parameters of the system. Therefore we empirically selected the parameter values that best fit the data. Contrast, noise, and a point spread function (PSF) analysis are the focus of our image quality tests. As shown in Fig. 29, the contrast test consists in measuring the difference between the average pixel intensity of an arbitrarily selected homogeneous region and the pixels inside the rebar feature. The noise measurement consists in calculating the pixel intensity standard deviation of the homogeneous region. As illustrated in Fig. 29(b), in order to measure the effects of the system PSF, we observe the amplitude profile of a line across the rebar horizontal diameter. All images were globally normalized to the range $[0,1]$. 


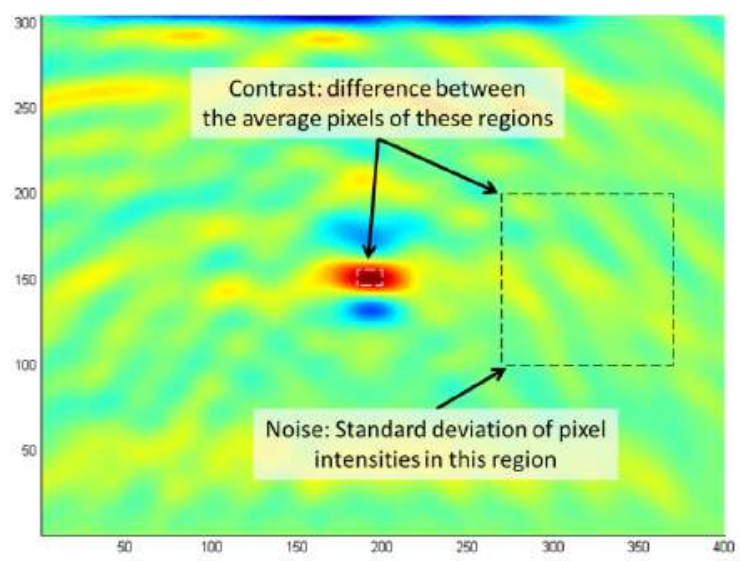

(a)

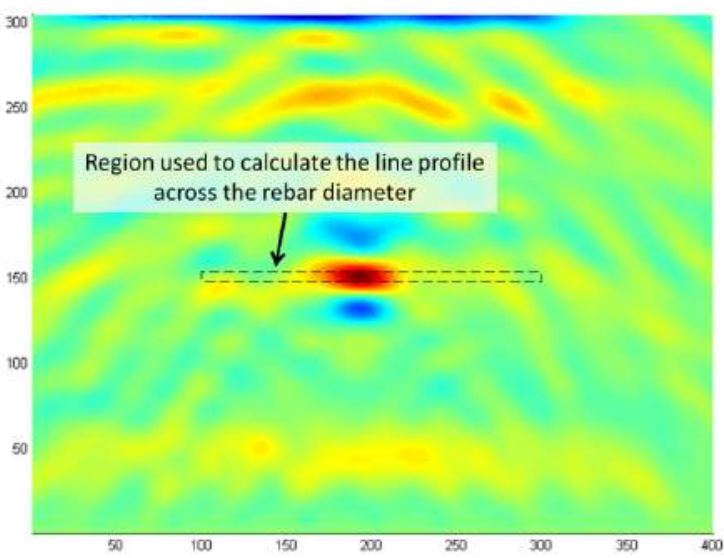

(b)

Fig. 29. Illustration of regions used for our quality tests: (a) contrast and noise and (b) PSF effects.

For the reconstructions in Fig. 29, the reconstruction space does not account for a couplant; therefore, the transducers are in full contact with the concrete surface (i.e., $d_{c}=0$ ). In order for the rebar to show up at the expected location (i.e., about $150 \mathrm{~mm}$ from the surface) the first 29 time samples (i.e., $29 \mu s$ ) were discarded. Figures 30-33 are TFM reconstructions, while Fig. 34 is a SAFT reconstruction. The reconstruction in Fig. 30 assumed a linear attenuation correction, while Figs. 31-33 assumed a non-linear model was assumed.

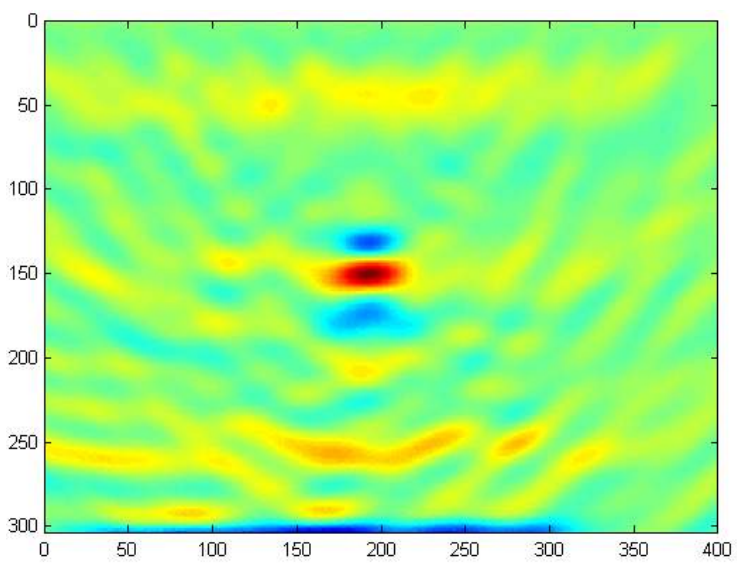

Fig. 30. TFM reconstruction with no attenuation correction.

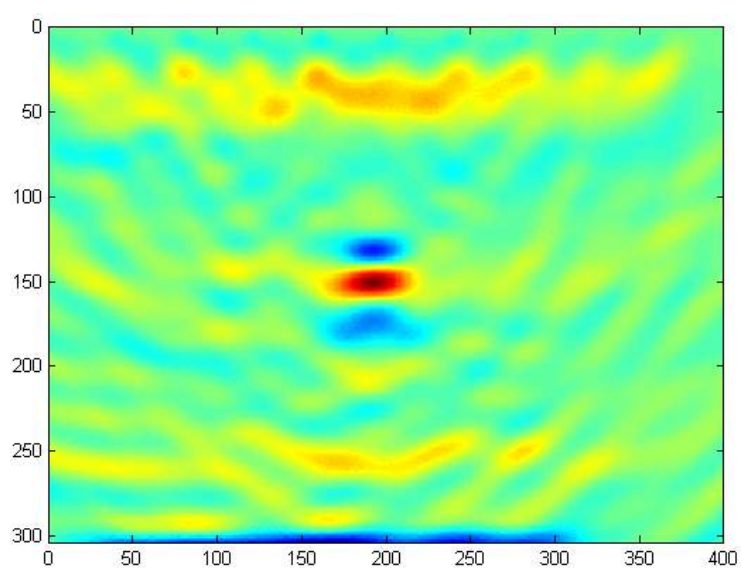

Fig. 31. TFM reconstruction with $\alpha=1.75$. 


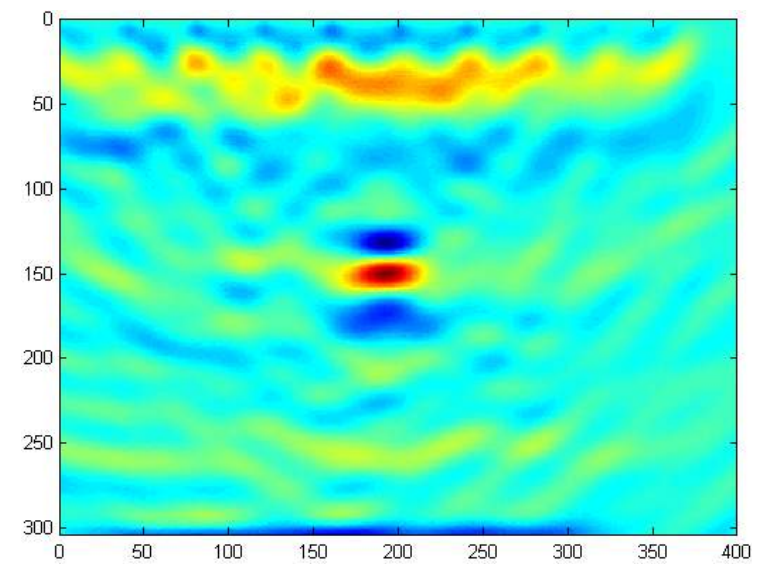

Fig. 32. TFM reconstruction with $\alpha=2.5$.

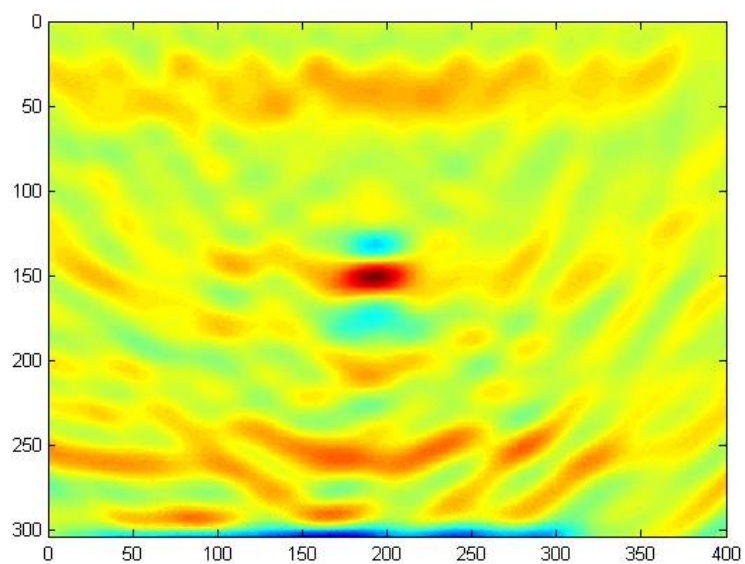

Fig. 33. TFM reconstruction with $\alpha=3.25$.

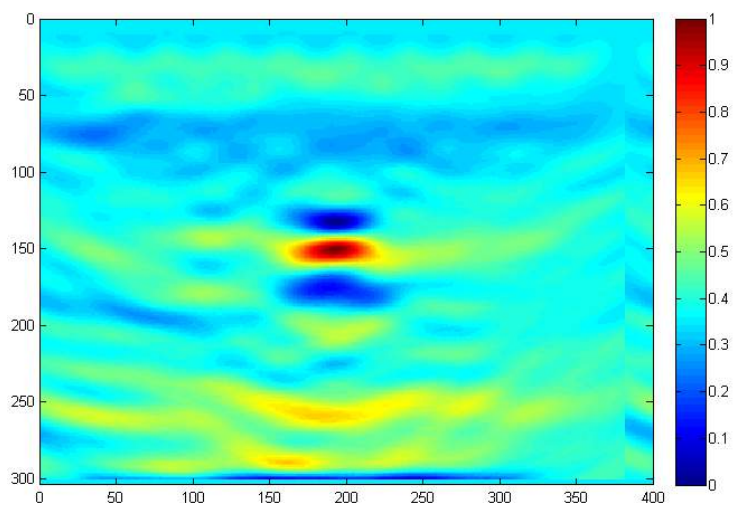

Fig. 34. SAFT reconstruction. 
The TFM reconstruction with $\alpha=2.5$ (Fig. 32) shows the best improvement with an $8 \%$ increase in contrast. The reconstructions with $\alpha=0$ (Fig. 30) and $\alpha=1.75$ (Fig. 31) show a contrast comparable to the SAFT reconstruction (Fig. 34). A contrast degradation of $19 \%$ is observed for the reconstruction with $\alpha=3.25$ (Fig. 33). For the noise test, the nonlinear correction where $\alpha=3.25$ showed the highest deviation in the homogeneous region, while the noise level in all other reconstructions were comparable to the SAFT noise levels. Figure 35(a) shows the horizontal line profile for the reconstructions in Figs. 30-34. After a global normalization of the reconstructions, a slight improvement in the system point spread function [i.e., narrower profile at Full Width at Half Maximum (FWHM)] is observed for the TFM linear and non-linear with $\alpha=2.5$ reconstructions. SAFT outperformed TFM for the other configurations. After a visual inspection of the reconstructions in Figs. 30-34, we determined that the strong artifacts at the transducer/media interface impede an appropriate normalization of the rebar region. After a local normalization of the marked region in Fig. 36, the rebar line profiles for TFM [Fig. 35(b)] show better contrast and a narrower PSF. The artifacts at the transducer/media interface could be suppressed by better estimation of a priori parameters (e.g., couplant gap, acoustic speed, attenuation coefficient, etc.).

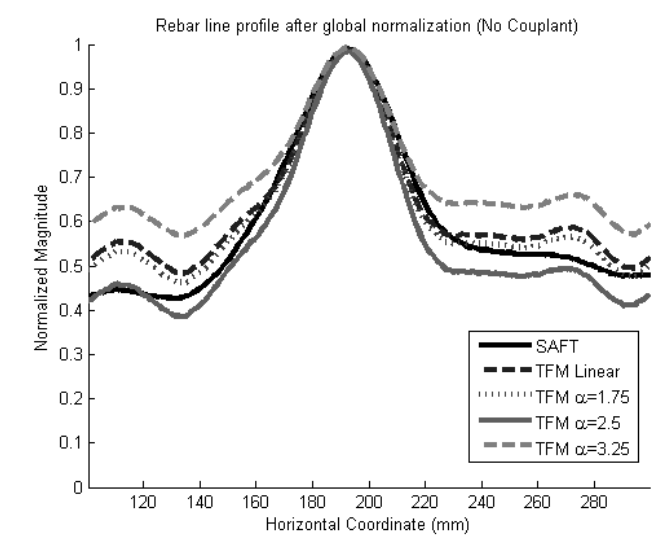

(a)

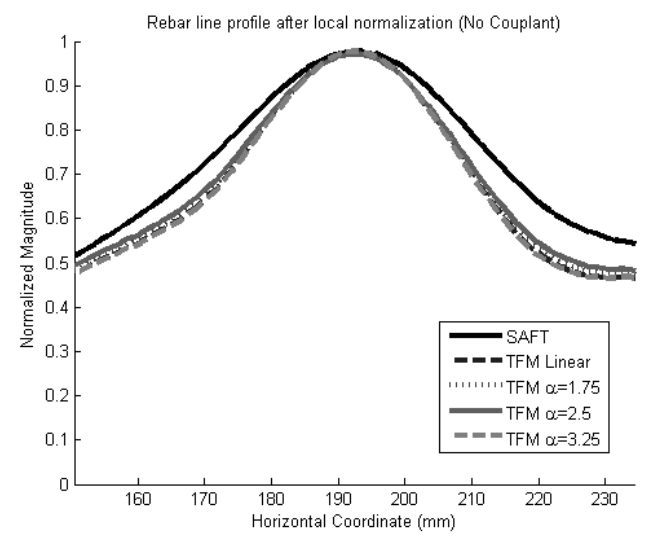

(b)

Fig. 35. Rebar line profiles assuming transducers in full contact with concrete surface. Results after (a) global and (b) local normalizations.

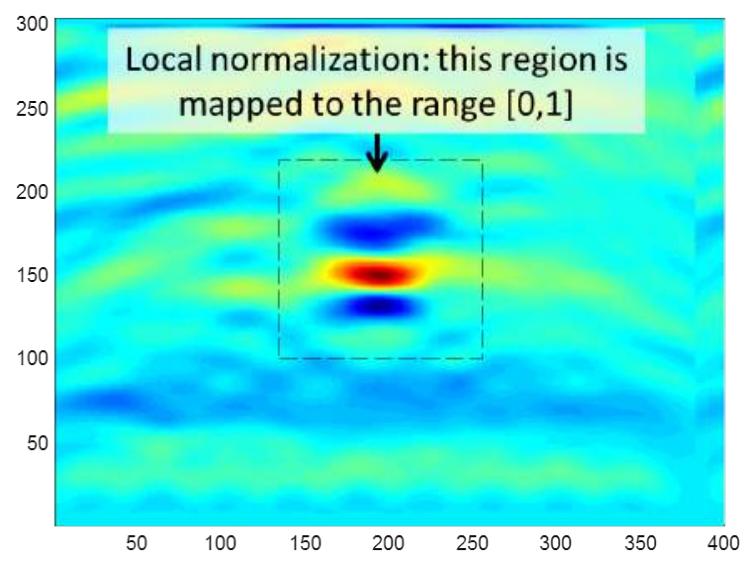

Fig. 36. Illustration of region used for local normalization. 
Figure 37(a) and 37(b) are TFM reconstructions with linear and non-linear $(\alpha=2.5)$ attenuation correction and an air couplant $\left(\mathrm{d}_{c}=5 \mathrm{~mm}\right)$ included to the reconstruction space. Such features cannot be added to the SAFT model; therefore, we compare the TFM with couplant reconstructions to the original SAFT reconstruction (Figs. 34 and 37(c)).

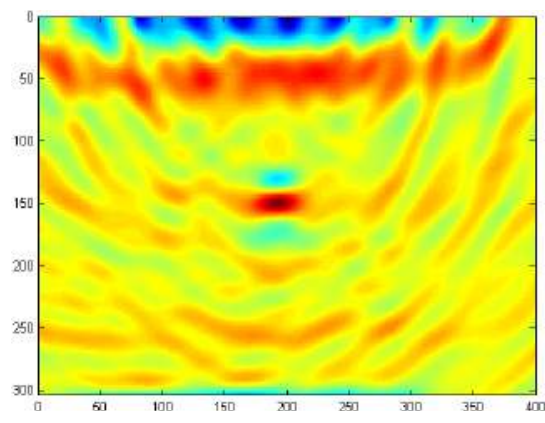

(a)

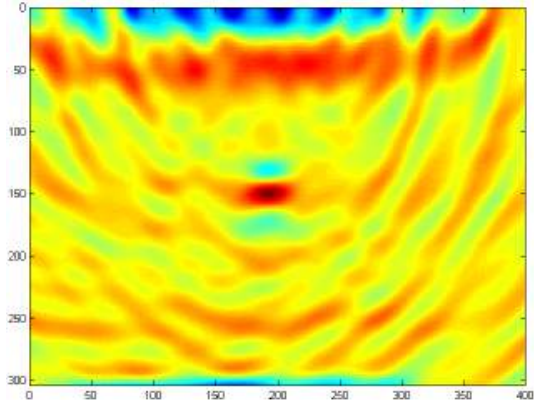

(b)

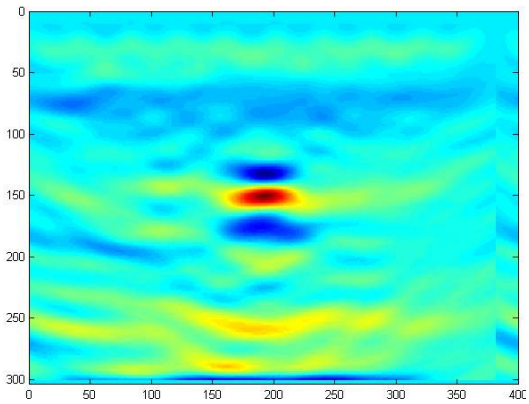

(c)

Fig. 37. Normalized image reconstructions assuming a couplant thickness $d_{c}=5 m m$. (a) TFM reconstruction with (a) linear and (b) non-linear $(\alpha=2.5)$ attenuation correction and (c) SAFT reconstruction.

There is no significant difference between the linear and nonlinear TFM reconstructions. Observe that the artifacts in the air/concrete interface (top of image) are stronger than those in the non-couplant case. These artifacts lower TFM reconstruction contrast by $20 \%$. In addition, noise is higher in the TFM reconstructions. Therefore, adding a couplant space degraded the quality of the reconstruction for this sample. The sample was reconstructed for different couplant gaps producing similar results. We cannot conclude that there is no gap between the transducers and the media from these results since for any two solids in contact there is a couplant gap unless both solids surfaces are perfectly flat and smooth. Consequently even for a spring-loaded transducer, such as in the MIRA systems, there is a gap at the transducer/concrete interface since the ultrasound generation element (i.e., the moving part) cannot directly touch the concrete. Without knowing the exact type of ceramic cover and its thickness, the TFM couplant parameter is of limited benefit. Attempts to receive this information from Acoustic Control Systems have been unsuccessful to date. Even though several commonly used ceramics were explored, we did not find a good match. However, these results emphasize the importance of estimating any couplant gap at imaging time.

Figure 38(a) shows the horizontal line profile of the rebar feature for the reconstructions in Fig. 37 after a global normalization. Again, the curves confirm the previous contrast measurements and show no significant improvement in the system point spread function. Although SAFT outperformed TFM for a global normalization, a local normalization of the rebar region shows that TFM provides more information about the rebar structure by narrowing the shape of the PSF and slightly increasing contrast. 
Repeatedly, our results indicate that for the TFM image formation a model is necessary to know accurately the a priori variables in order to take advantage of the extra features offered by this new algorithm.

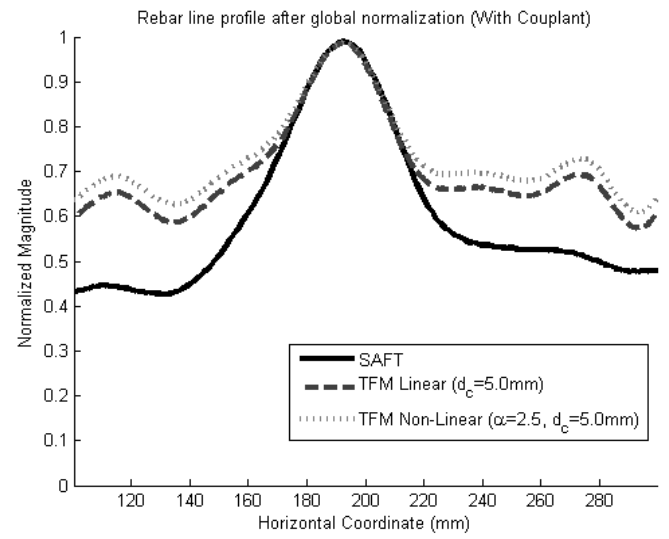

(a)

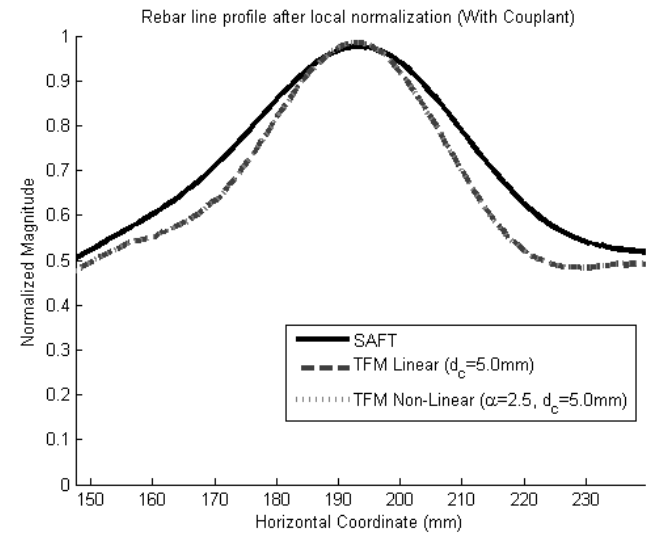

(b)

Fig. 38. Rebar line profiles assuming a couplant gap of $5 \mathrm{~mm}$. Results after (a) global and (b) local normalizations.

\subsection{NEXT STEPS FOR TFM}

Typically the quality obtained from a reconstruction algorithm depends on the quality of the image formation model. Although previous work has shown the superiority of TFM over SAFT, TFM's advantage depends on an accurate estimation of a priori variables. Our results show that there is a slight improvement for TFM reconstructions relative to SAFT, even when the acoustic properties were unknown for the ultrasonic system, the couplant, and the media. We expect major overall improvements of a priori variables are better defined. Therefore, the next evident step is to characterize the transducers of the MIRA v1 and v2 systems and develop software tools to estimate the media acoustic properties from the measured signals - as it is currently done for estimating the acoustic speed of the media. TFM can also be improved by including new correction parameters, such as phase correction that can be integrated into the current TFM implementation. Additionally the current corrections will benefit from a better characterization of the ultrasonic system. An area that needs improvement is reconstruction time for samples with a gap between transducers and the media. At full resolution $(1 \mathrm{~mm} /$ pixel $)$, reconstructing a single pixel takes $45 \mathrm{~ms}$ in the current Matlab implementation, which results in about $1.5 \mathrm{hr}$ for each of the reconstruction images shown above. There are several alternatives to reduce execution time, such as exporting the implementation to the $\mathrm{C} / \mathrm{C}++$ programming languages, vectorization, parallel processing, and GPU processing. By implementing some of the above execution time reduction techniques, it is believed that processing times for images could be reduced to approximately 2 minutes per image, which is considered manageable. 


\section{POTENTIAL FUTURE IMPROVEMENTS}

\subsection{AUTO FOCUSING}

Auto-focusing, as applied to NDE inspection, is an algorithm developed for dealing with the unknown surface geometry of flexible ultrasonic arrays [12]. In SAFT and TFM, the calculations are made with the assumption that it is a flat planar ultrasonic array, but the array may not actually be flat. The variations in the surface being inspected using the array are directly translated to the profile of the array. This situation is highly applicable to the spring-loaded dry point contacts of the MIRA system. In the MIRA's situation, the distance between the surface and the transducers is not uniform due to both variations in the concrete surface and the amount of compression of the spring loaded-contacts. The autofocusing technique has been further extended in a step towards full model-based image reconstruction $[13,14]$. The model-based auto-focusing algorithm uses a priori information regarding a known feature, such as the back wall reflection, as part of an error metric calculation. The positions of the array elements are optimized with the goal being to minimize the error between the actual back wall reflection and the model of the back wall reflection.

Currently, the published research using these algorithms has shown their abilities for focusing/correcting TFM image reconstructions in situations where the ultrasonic transducer passes over a weld and as a means to allow real-world use of flexible ultrasonic arrays for the inspection of complex structures $[12,13,14]$. In the case of the weld inspections, the weld filler material bulges above the surface of the welded object's typical surface and causes the distance the propagating wave travels to change. This causes a loss in focus, resolution, and readability for the area where the transducer went over the weld bulge until it is flat on the object's surface again.

A similar problem faces the inspection of concrete structures. In the case of a containment structure, the surface the instrument is pressed against is typically curved, not flat. Dry-point contact transducers contain a spring which helps ensure reliable contact with the surface. However, the degree that each contact is depressed is unknown and varies as the instrument is repositioned during the process of a scan. Therefore, the assumption that every element of a transducer array is at the same height as the rest of the elements is not sustainable or realistic in the long term. The same uncertainty applies to the profile of both the front wall and back wall surfaces being inspected. This is especially true in the case of a curved nuclear containment structure and cooling towers.

Until a full model-based reconstruction method is developed and validated against known test structures, the implementation of an auto-focusing algorithm for use in the inspection of concrete structures using ultrasonic arrays will be of benefit. It provides a meaningful bridge between the current SAFT and TFM implementations and the current push in image reconstruction algorithms toward modelbased image reconstruction methods. 


\subsection{PHASE}

From previous projects at ORNL we have found that the phase information of a signal contains valuable information and often avoids some of the downfalls that the signal amplitude is subject to (SAFT and TFM directly use the amplitudes of the received signals). A groundbreaking journal article was published in 2008 on the use of phase information for defect and structure identification in concrete [15]. This article made use of the Hilbert transform to generate the phase information from the non-complex sampled received signals of an ultrasonic array. We believe that by building an instrument similar to the MIRA unit, but with the ability to digitize the received signals amplitude and phase, we can improve the detection and identification of the internal structure of complex concrete objects. The capabilities exhibited in the journal articles on the mathematical generation of phase information provide enough proof and motivation to develop a system capable of acquiring the true phase of the reflected ultrasound waves.

\subsection{MBIR}

TFM has a slight edge over SAFT because of its better image formation model. Nevertheless, TFM is still a delay-and-sum approach, which lacks an advanced framework to intelligently back-propagate the measured amplitudes to the correct reconstruction voxel. Sound waves can be modeled as rays; therefore, we can import state-of-the-art techniques from the $\mathrm{x}$-ray and neutron imaging fields to ultrasound imaging. A technique with great potential for ultrasound reconstruction is the Model-Based Iterative Reconstruction (MBIR) technique [16]. MBIR was designed for high-dimensional problems, a great fit to data intense ultrasound signals. The fundamental idea behind MBIR is to solve the optimization problem

$$
\dot{x}=\operatorname{argmin}_{x}\left\{\frac{1}{2}\|y-A x\|_{\Lambda}^{2}+u(x)\right\},
$$

where $A$ is the system matrix modeling the media interior and the ultrasound system, $y$ is a vector with the reflection measurements for every transducer, $x$ is a vector with the media acoustic property to reconstruct (e.g., acoustic speed, Intensity Reflectivity Coefficients), and $u(x)$ is a priori used to emphasize particular solutions. For example, the contrast for the edges in the object to reconstruct can be enhanced with the right priori selection. The reconstruction process starts with an initial assumption of the media interior-an object estimate. A convergence algorithm like the Conjugate Gradient is used to refine the estimated object from the measured data until some stopping criteria or a number of iterations are reached. In contrast to TFM, where the measured data is distributed across the media based on time delays alone, with MBIR the space of objects is searched in order to find the optimal acoustic configuration that fits the data. Although the immediate impact of MBIR on ultrasound reconstruction will be to increase signal-to-noise ratio (SNR) due to a better modeling of the hardware and the physics of sound, the principal advantage of this technique is that the pixel intensities in the reconstruction image will correlate to the acoustic properties of the imaged material, removing the need for a trained expert to interpret the reconstructions. 


\section{CONCLUSIONS}

Using ultrasonic array data collected from three specimens of varying complexity, this report illustrates the benefits of using time-frequency analysis with Synthetic Aperture Focusing Technique (SAFT). While SAFT is an image reconstruction technique commonly used in conjunction with ultrasonic arrays, decomposing the original ultrasound signals into various frequency bands does render more definitive results. By using wavelet packet decomposition (a computational efficient method), the selected frequency bands are reconstructed into a time-series dataset that can be processed by known analytical techniques. Additionally, this report briefly examines the benefits of the Total Focusing Method (TFM) as compared to SAFT. While the TFM does yield slightly better results, computations are greatly increased. While the three specimens used in this report are not representative of NPP concrete structures, these specimens do allow a comparison of the analysis methods explored.

Three additional possible improvements are briefly explored: Auto Focusing, Utilization of Phase Information, and Model Based Image Reconstruction (MBIR). These methods should be further explored if the results of the time-frequency analysis with SAFT on a thick specimen, currently being designed and expected to be constructed in early FY 2015, are inadequate to identify the embedded flaws of the thick specimen. 



\section{REFERENCES}

1. D. Clayton, C. Smith, C. C. Ferraro, J. Nelson, L. Khazanovich, K. Hoegh et al., Evaluation of Ultrasonic Techniques on Concrete Structures, ORNL/TM-2013/430, Oak Ridge, TN (2013).

2. C. Gargour, M. Gabrea, V. Ramachandran, and J.-M. Lina, "A short introduction to wavelets and their applications," Circuits and Systems Magazine, 9(2), 57- 68 (June 2009).

3. O. Rioul and M. Vetterli, "Wavelets and signal processing," Signal Processing Magazine, 8(4), 1438 (October 1991).

4. I. Daubechies, Ten Lectures on Wavelets. Philadelphia, PA, USA: Society for Industrial Applied Mathematics (SIAM), 1992.

5. ASTM, Standard Test Method for Pulse Velocity Through Concrete, West Conshokocken: ASTM International, 2009.

6. K. Hoegh, Ultrasonic Linear Array Evaluation of Concrete Pavements, Ph.D. Thesis, University of Minnesota, 2013.

7. Germann Instruments, MIRA Specification Sheet, 2014. Retrieved from http://www.germann.org/TestSystems/MIRA Tomographer/MIRA Tomographer.pdf

8. D. R. Hiltunen, D. Algernon, and C. C. Ferraro, Validation of Nondestructive Testing Equipment for Concrete, BD545-80, Gainesville: Florida Department of Transportation Research Center, 2010.

9. T. L. Szabo, Diagnostic Ultrasound Imaging: Inside Out (2nd ed.). Oxford, UK: Academic Press, 2014.

10. B. Drinkwater and P. Wilcox, "Ultrasonic arrays for non-destructive evaluation: A review," NDT\&E International, 39, 525-541 (2006).

11. J. Zhang, B. Drinkwater, and P. Wilcox, "Comparison of Ultrasonic Array Imaging Algorithms for Nondestructive Evaluation," in Trans. Ultrasonics, Ferroelectrics and Frequency Control, IEEE, 60(8), 17321745 (2013).

12. A. J. Hunter, B. W. Drinkwater, and P. D. Wilcox, "Autofocusing ultrasonic imagery for nondestructive testing and evaluation of specimens with complicated geometries," NDT\&E International, 43(2), 78-85 (2010).

13. A. J. Hunter, B. W. Drinkwater, and P. D. Wilcox, "Least-squares estimation of imaging parameters for an ultrasonic array using known geometric image features," IEEE Transactions on Ultrasonics, Ferroelectrics and Frequency Control, 58(2), 414-426 (February 2011).

14. A. J. Hunter, B. W. Drinkwater, and P. D. Wilcox, "A Model Based Autofocus Algorithm for Ultrasonic Imaging Using a Flexible Array," pp. 863-870 in Review of Progress in Quantitative Nondestructive Evaluation (QNDE), Kingston, RI, USA: AIP Conference Proceedings (2009).

15. K. Mayer, L. Karl-Jörg, M. Krause, B. Milmann, and F. Mielentz, "Characterization of Reflector Types by Phase-Sensitive Ultrasonic Data Processing and Imaging," Journal of Nondestructive Evaluation, 1-3, 35-45 (2008).

16. R. Zhang, J.-B. Thibault, B. Charlie, K. D. Sauer, and J. Hsieh, "Model-Based Iterative Reconstruction for Dual-Energy X-Ray CT Using a Joint Quadratic Likelihood Model," IEEE Transactions on Medical Imaging, 33(1), 117-134 (2014). 



\section{APPENDIX A. INTERPRETATION AND ANALYSIS OF ULTRASONIC LINEAR ARRAY SIGNALS}





\section{APPENDIX A. INTERPRETATION AND ANALYSIS OF ULTRASONIC LINEAR ARRAY SIGNALS}

This appendix describes the development of various reconstruction procedures for interpretation of the ultrasonic linear array signals. In addition, several application-specific automation procedures were developed to increase productivity and accuracy in interpretation of a large number of reconstructions.

\section{A.1 RECONSTRUCTION}

Typical synthetic aperture radar (SAR) and synthetic aperture focusing technique (SAFT) formulations are applied for interpretation of multiple signals with sending and receiving transducers at the same location. These formulations need to be generalized for interpretation of signals with sending and receiving transducers located at different locations for application with MIRA. In this case, a signal sent from one location but received at various locations along the surface should be accounted for in this formulation.

Similar to the formulation for point source emission and reception at a single location, the region of interest (ROI) below the testing aperture can be treated as a collection of point targets. The fundamental expression for SAR is generalized for each emitting and receiving transducer pair positioned at $x_{e}^{\prime}$ and $x_{r}^{\prime}$, respectively, and can be represented as shown in Eq. (A.1)]:

$$
s\left(x_{e}^{\prime}, x_{r}^{\prime}, t\right)=\int_{x}^{\cdot} \int_{z} f(x, z) * \delta\left(t^{*}\left(t, x_{e}^{\prime}, x_{r}^{\prime}, x, z\right)\right) d z d x
$$

where $s\left(x_{e}^{\prime}, x_{e}^{\prime}, t\right)$ is the received impulse due to emitted impulse $\delta\left(t^{*}\left(t, x_{e}^{\prime}, x_{r}^{\prime}, x, z\right)\right) ; f(x, z)$ is the reflectivity function of the ROI; $x$ and $z$ are the horizontal and vertical positions in the ROI, respectively; and $t^{*}$ is defined by the relationship

$$
t^{*}=t-\frac{1}{c}\left(\sqrt{z^{2}+\left(x-x_{e}\right)^{2}}+\sqrt{z^{2}+\left(x-x_{r}\right)^{2}}\right) .
$$

The impulse response received at the surface as a function of time, $s\left(x_{e}^{\prime}, x_{r}^{\prime}, t\right)$, is a combination of reflections from each position below the measurement as defined by the reflectivity function, $f(x, z)$. This function depends on the reflection coefficient of each of the potential point targets in the ROI, which is a function of changes in modulus and density. Since the reflection coefficients are determined by changes in acoustic impedance characteristics and thus contain the information about material changes in stiffness or density, this is the information of interest when creating the reconstruction images. Figure A.1 shows a representation of potential contributing point sources for a single intensity value within $s\left(x_{e}, x_{r}, t\right)$ according to the fundamental SAR equation. 


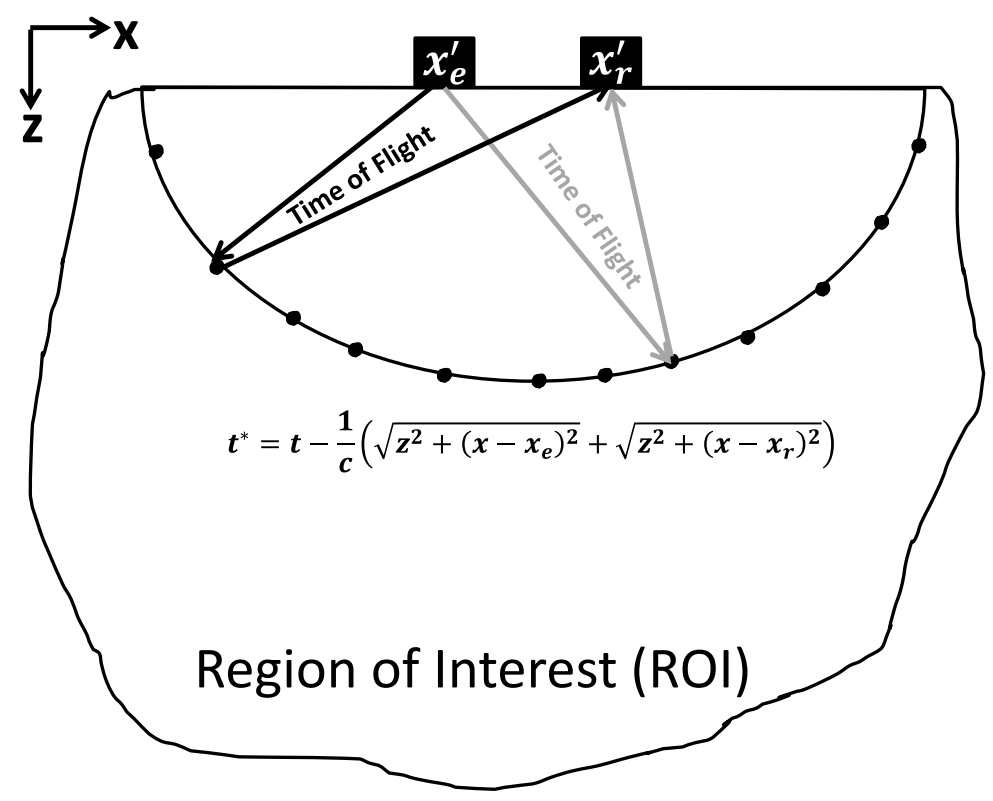

Fig. A.1. Representation of potential contributing point sources at a constant time (Roundtrip) from the emitting/receiving transducer according to the fundamental expression.

\section{A.2 SAFT B-SCAN}

The synthetic aperture focusing technique can be used for the ultrasonic linear array used in this study in a similar manner to that described for single sending and receiving pairs. To allow for integration over the various transducer locations, $\int_{x_{e}^{\prime}}^{\cdot} d x_{e}^{\prime} \int_{x_{r}^{\prime}} \ldots d x_{r}^{\prime}$, the impulses received at the surface versus time can be expressed in terms of distances. This can be accomplished by assuming a constant shear wave velocity, $C_{S}=C_{S}^{A v g}$, determined from Eq. (A.1). If the signals are emitted within interval $\left[x_{\text {emin }}^{\prime}, x_{e m a x}^{\prime}\right]$ and received within interval $\left[x_{r m i n}^{\prime}, x_{r \max }^{\prime}\right]$, the reconstructed image at each point $\widehat{\boldsymbol{o}}(x, z)$ can be obtained by integrating over all possible transducer pair (impulse emit and receive) locations:

$\widehat{\boldsymbol{o}}(x, z)=\int_{x_{\text {emin }}^{\prime}}^{x_{\text {emax }}^{\prime}} d x_{e}^{\prime} \int_{x_{r \text { min }}^{\prime}}^{x_{r \max }^{\prime}} \mathrm{A}\left(x_{r}^{\prime}, x_{e}^{\prime}, x, z\right) * s\left(x_{r}^{\prime}, x_{e}^{\prime}, \frac{1}{c}\left(\sqrt{z^{2}+\left(x-x_{e}^{\prime}\right)^{2}}+\sqrt{z^{2}+\left(x-x_{r}^{\prime}\right)^{2}}\right)\right) d x_{r}^{\prime}$,

where $\mathrm{A}\left(x_{r}^{\prime}, x_{e}^{\prime}, x, z\right)$ is the apodization factor that is typically a function of the distance traveled, incident angle, and/or divergence of the reflection point location with respect to the emitted/received signal.

The measured signals for the setup in this study have $T=10$ transducer locations at spacing $\Delta x^{\prime}=40 \mathrm{~mm}$. Figure A.2 shows an example linear array setup with $x_{1}^{\prime}$ being the leftmost transducer. For the ultrasonic linear array used in this study, the leftmost emitting transducer is located at the leftmost transducer location, $x_{e 1}^{\prime}=x_{1}^{\prime}$, the leftmost receiving transducer is located adjacent to the leftmost emitting transducer $x_{r 1}^{\prime}=x_{e 1}^{\prime}+\Delta x=x_{e 1+1}^{\prime}$, the rightmost receiving transducer is located at the rightmost transducer location $x_{R}^{\prime}=x_{T}^{\prime}$, and the rightmost emitting transducer is located adjacent to the rightmost receiving transducer location $x_{E}^{\prime}=x_{R}^{\prime}-\Delta x=x_{T-1}^{\prime}$. 


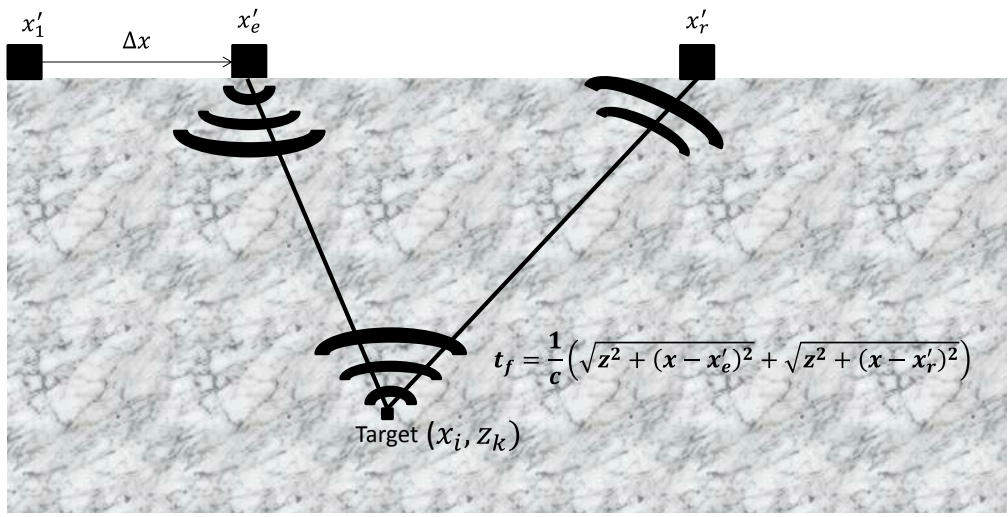

Fig. A.2. Linear array representation.

After applying the shift factor to account for system delay, the reconstructed image is obtained at discrete points by summing over all possible transducer pair locations using the following relationship for the ultrasonic linear array:

$$
\hat{o}_{i, k}=\sum_{e=1}^{T-1} \sum_{r=e+1}^{T} \mathrm{~A}\left(x_{r}, x_{e}, x_{i}, z_{k}\right) \Psi_{e, r}\left(x_{i}, z_{k}\right),
$$

where

$$
\Psi_{e, r}\left(x_{i}, z_{k}\right)=s\left(x_{r}, x_{e}, \frac{1}{c}\left(\sqrt{z_{k}^{2}+\left(x_{i}-x_{e}\right)^{2}}+\sqrt{z_{k}^{2}+\left(x_{i}-x_{r}\right)^{2}}\right)\right),
$$

where $\widehat{\boldsymbol{o}}_{i, k}$ is the image reflectivity assigned to each position within the ROI, $T$ is the number of transducer locations, $e$ and $r$ are the indexes for the emitting and receiving transducers, and $i$ and $k$ are the indexes for the horizontal and vertical positions of the ROI.

If the ROI has $\mathrm{W}$ indexes in the horizontal direction and D indexes in the vertical direction, the following

\begin{tabular}{|c|c|c|c|c|c|}
\hline & $\widehat{\boldsymbol{o}}_{1, \mathbf{1}}$ & $\widehat{o}_{2,1}$ & $\ldots$ & $\ldots$ & $\widehat{o}_{W, 1}$ \\
\hline & $\widehat{\boldsymbol{o}}_{1,2}$ & .... & $\ldots$ & $\ldots$ & $\ldots$ \\
\hline$=$ & $\ldots$ & .... & $\widehat{\boldsymbol{O}}_{i, \boldsymbol{k}}$ & $\ldots$ & $\ldots$ \\
\hline & $\ldots$ & $\ldots$ & $\ldots$. & $\ldots$ & $\ldots$ \\
\hline & $\widehat{\boldsymbol{O}}_{1, D}$ & .... & .... & ... & $\widehat{\boldsymbol{o}}_{W, D}$ \\
\hline
\end{tabular}
matrix formalization can be used to represent SAFT reconstructed image, $[\widehat{\boldsymbol{O}}]$ : 
The apodization factor accounts for incident angle and other traits of the signal and tested medium. For the ultrasonic linear array used in this study for testing of concrete, the apodization factor given in Eq. (A.7) was used:

$$
\mathrm{A}\left(x_{r}^{\prime}, x_{e}^{\prime}, x_{i}, z_{k}\right)=\alpha_{e}\left(x_{e}^{\prime}, x_{i}, z_{k}\right) * \alpha_{r}\left(x_{r}^{\prime}, x_{i}, z_{k}\right)
$$

where

$$
\alpha_{\mathrm{e}}\left(\mathrm{x}_{\mathrm{e}}^{\prime}, \mathrm{x}_{\mathrm{i}}, \mathrm{z}_{\mathrm{k}}\right)=\frac{\mathrm{z}_{\mathrm{k}}}{\sqrt{\left(\mathrm{x}_{\mathrm{i}}-\mathrm{x}_{\mathrm{e}}^{\prime}\right)^{2}+\mathrm{z}_{\mathrm{k}}^{2}}}
$$

and

$$
\alpha_{\mathrm{r}}\left(\mathrm{x}_{\mathrm{e}}^{\prime}, \mathrm{x}_{\mathrm{i}}, \mathrm{z}_{\mathrm{k}}\right)=\frac{\mathrm{z}_{\mathrm{k}}}{\sqrt{\left(\mathrm{x}_{\mathrm{i}}-\mathrm{x}_{\mathrm{r}}^{\prime}\right)^{2}+\mathrm{z}_{\mathrm{k}}^{2}}}
$$

An example of typical data from the ultrasonic linear array on a reinforced concrete pavement (CRCP) is given herein. Figure A.3 shows a schematic of the CRCP structure at the example scan location. It can be observed that there is a set of three longitudinal reinforcements represented by black circles in the schematic within the ROI where the measurement was taken.

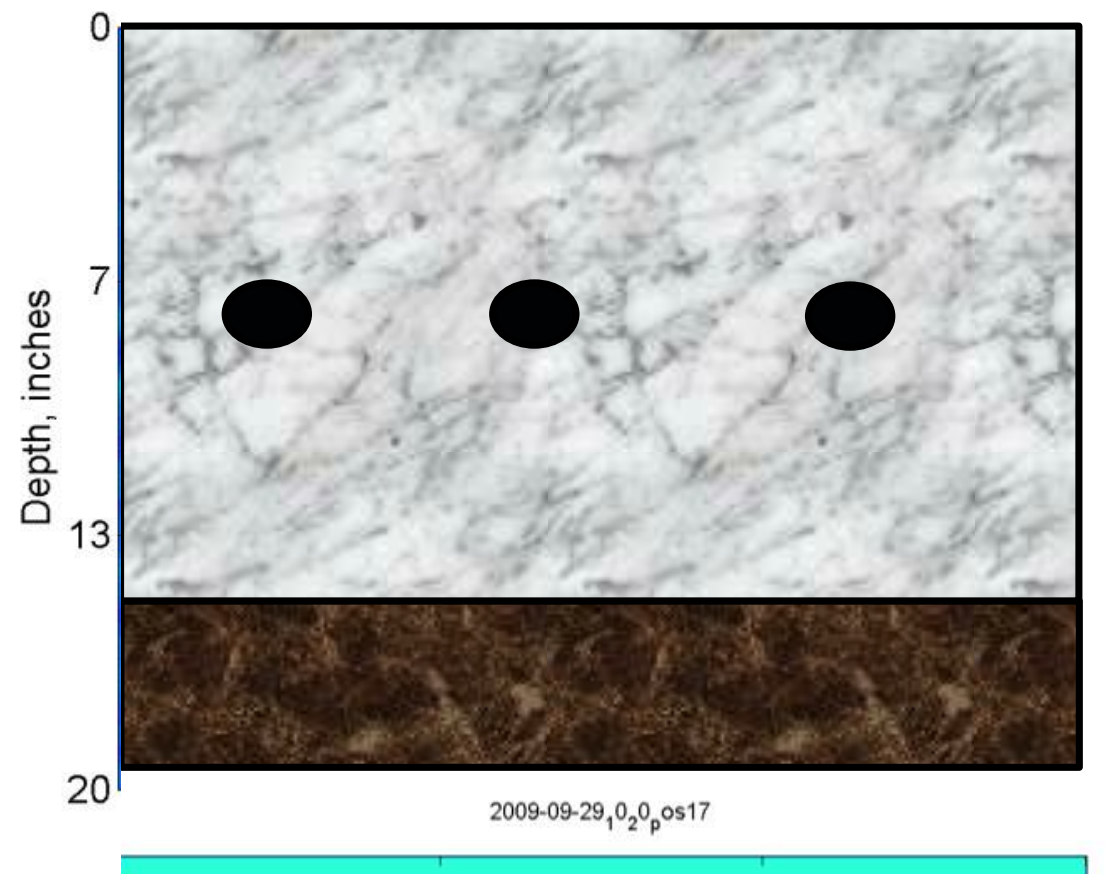

Fig. A.3. Schematic of the CRCP subsurface at the example scan location.

Figure A.4 shows three example impulse responses, $\Psi_{1,6}(t), \Psi_{2,5}(t), \Psi_{3,4}(t)$, of the total 45 emitting and receiving pairs comprising the example scan. It can be observed that there are peaks in amplitude at certain locations within the time histories in addition to the direct arrival positions. While these spikes in amplitude are associated with reflectivity within the ROI, such as reinforcements and layer boundaries, the locations of the cause of these reflections are difficult to interpret. 


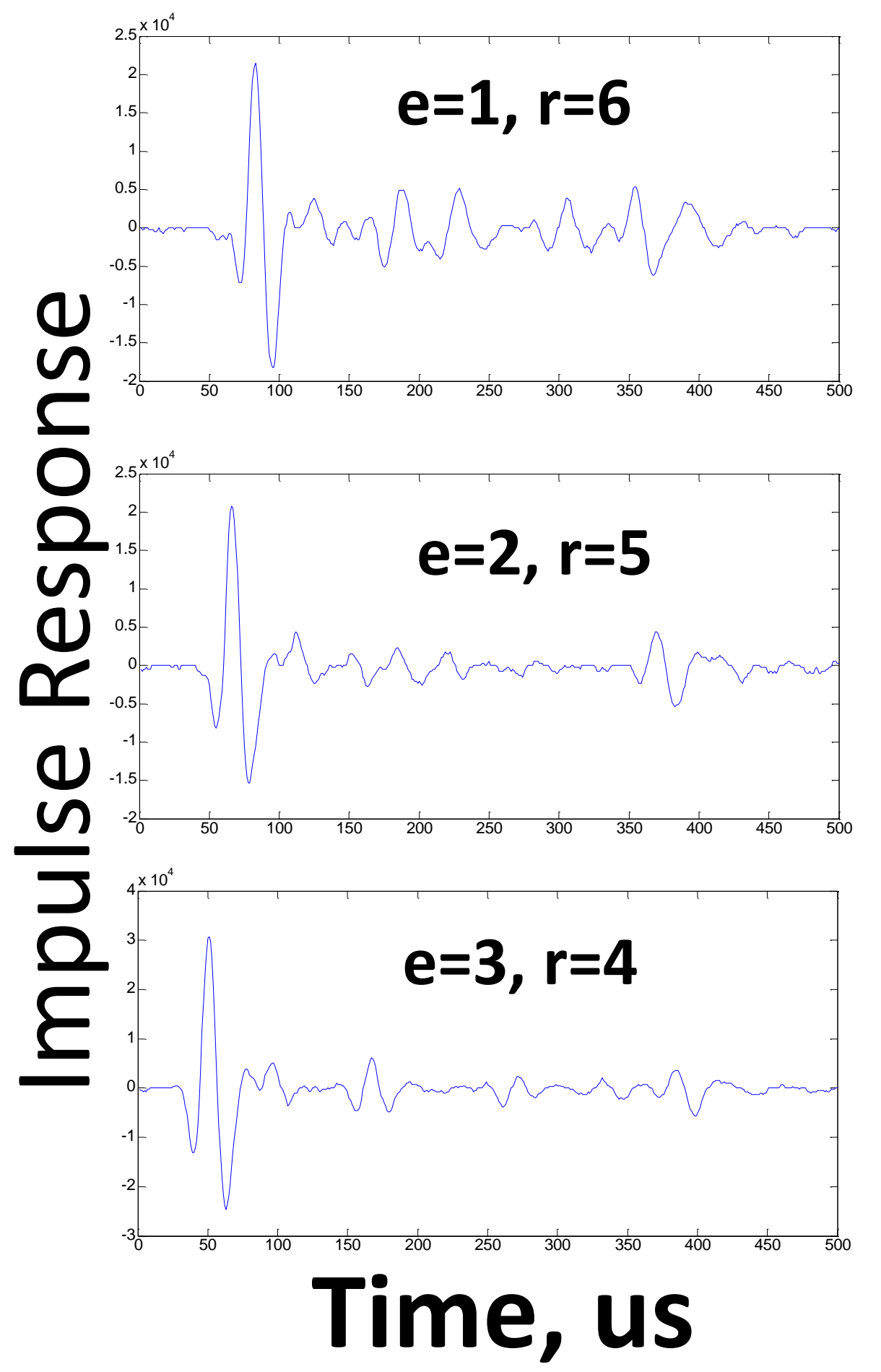

Fig. A.4. Example emitting-receiving pairs from an ultrasonic linear array scan.

Figure A.5 shows the resulting SAFT reconstructions after using Eq. (A.4) to reconstruct all 45 impulse pairs, $\Psi_{e, r}(t)$. Figure A.5(a) was reconstructed using an apodization factor of 1, and the apodization factor defined in Eq. (A.7) was used in Fig. A.5(b). Both reconstructions give more information about the subsurface of the structure than the individual impulses. 
The high reflectivity locations in the SAFT reconstructions indicate the location of changes in acoustic impedance such as the reinforcements and the layer boundary. It can also be observed that the structural noise observed in Fig. A.5(a) in the sound concrete portions is not present in Fig. A.5(b). This illustrates that the use of the apodization factor defined in Eq. (A.7) improves the focusing capabilities of the SAFT reconstruction and eliminates some of the structural noise such as direct arrival intensities that do not represent the reflectivity of the ROI.
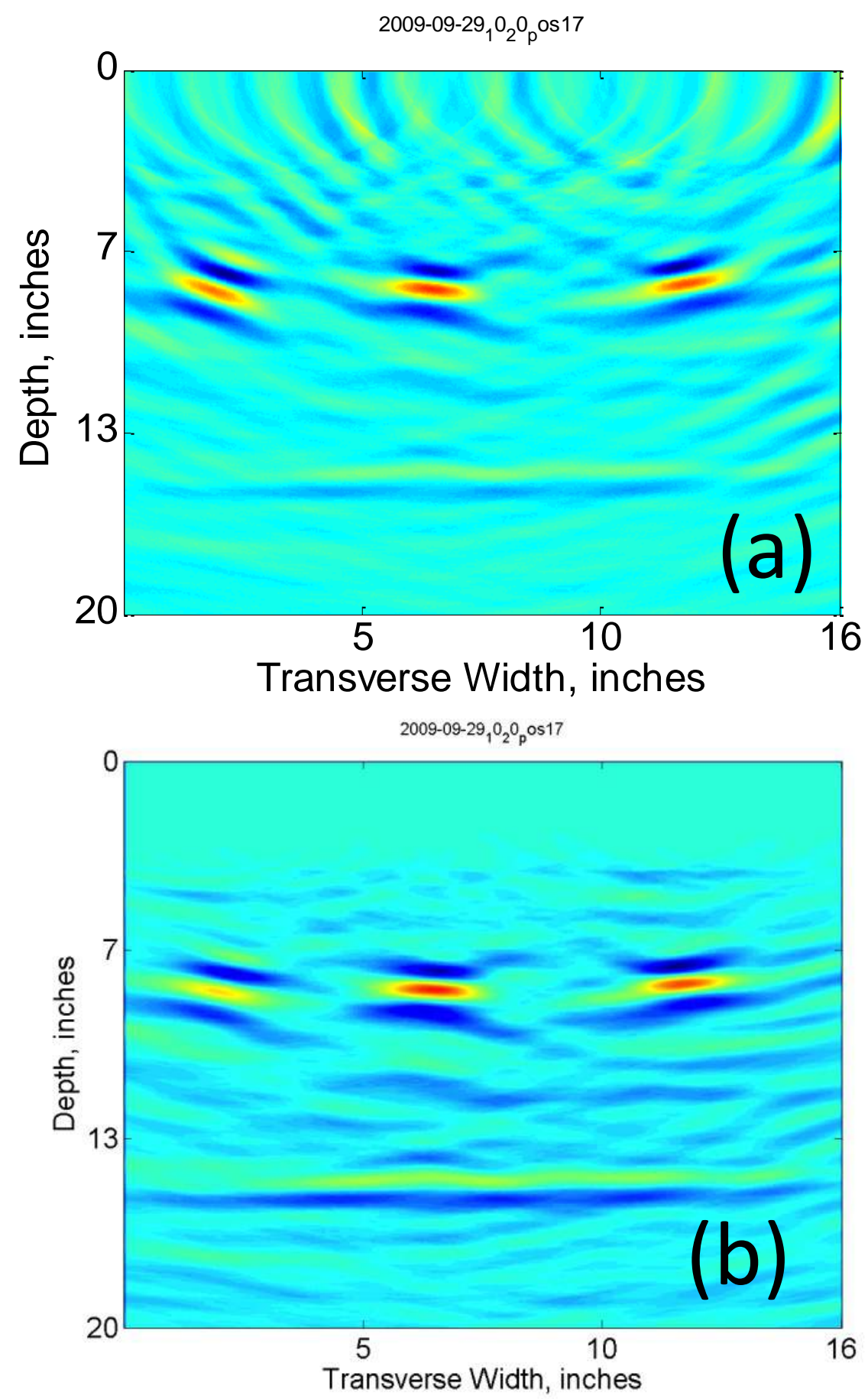

Fig. A.5. Example SAFT reconstruction with the apodization factor equal to (a) 1 and (b) defined by Eq. (A.7). 


\section{A.3 INSTANTANEOUS AMPLITUDE SAFT B-SCAN (SAFT-IA)}

In most cases, the reconstruction analysis for concrete pavement applications is concentrated on identifying the source of the changes in acoustic impedance in the subsurface. Further focusing of the reconstruction can be achieved by analyzing changes in instantaneous amplitude within the ROI. The Hilbert transform is useful in calculating instantaneous attributes of a time series, especially the amplitude and frequency [1-7]. The Hilbert transform, $\Upsilon(z)$, of a given function, $\chi(z)$, is defined by the following equation:

$$
\Upsilon(z)=\frac{1}{\pi} P \int_{-\infty}^{\infty} \frac{\chi(s)}{z-s} d s
$$

where $P$ is the principal value of the singular integral in Eq. (A.8).

And the complex analytic signal, $Z(z)$, is given by

$$
Z(z)=\chi(z)+j Y(z) .
$$

Since the reconstruction analysis in this study is based on focusing high reflectivity (changes in acoustic impedance) within the ROI with high-magnitude pulse amplitudes, calculation of instantaneous amplitudes during the SAFT analysis can give higher resolution reconstructions. Since the Hilbert Transform envelope will be used during the reconstruction process, a slight change in the determination of the shift factor must be applied. Equation (A.10) is used to calculate the instantaneous amplitude of each individual transducer pair impulse time history.

$$
\Psi_{e, r}^{I A}(t)=\sqrt{\left(\Psi_{e, r}(t)\right)^{2}+\left(\frac{1}{\pi} P \int_{-\infty}^{\infty} \frac{\Psi_{e, r}(s)}{t-s} d s\right)^{2}}
$$

where $\Psi_{e, r}^{I A}(t)$ defines the instantaneous amplitude envelope of time history pair, $\Psi_{e, r}(t)$, and $P$ is the principal value of the singular integral in Eq. (A.10).

Use of the instantaneous amplitude, $\Psi_{e, r}^{I A}(t)$, permits a modification of Eq. (A.5), which was based on the raw impulse time-history signal. The instantaneous amplitude-based shift factor, $t^{S H I F T, H i l b}$, for each signal is given in Eq. (A.11):

$$
t^{S H I F T, H i l b}=\frac{1}{E R} \sum_{e=1}^{T-S P^{\min }} \sum_{r=S P^{\min }}^{T} t_{e, r}^{M a x H}-\frac{x_{e, r}^{\prime}}{c_{S}^{A v g}},
$$

where $t_{e, r}^{\operatorname{MaxH}}$ is the time of flight to the maximum instantaneous amplitude of each $\Psi_{e, r}^{I A}$. Figure A.6 shows the same example transducer pair impulse response, $\Psi_{e, r}$, and corresponding instantaneous amplitude envelope, $\Psi_{e, r}^{I A}$, illustrating the process of obtaining $t_{e, r}^{\operatorname{MaxH}}$ (bottom). 

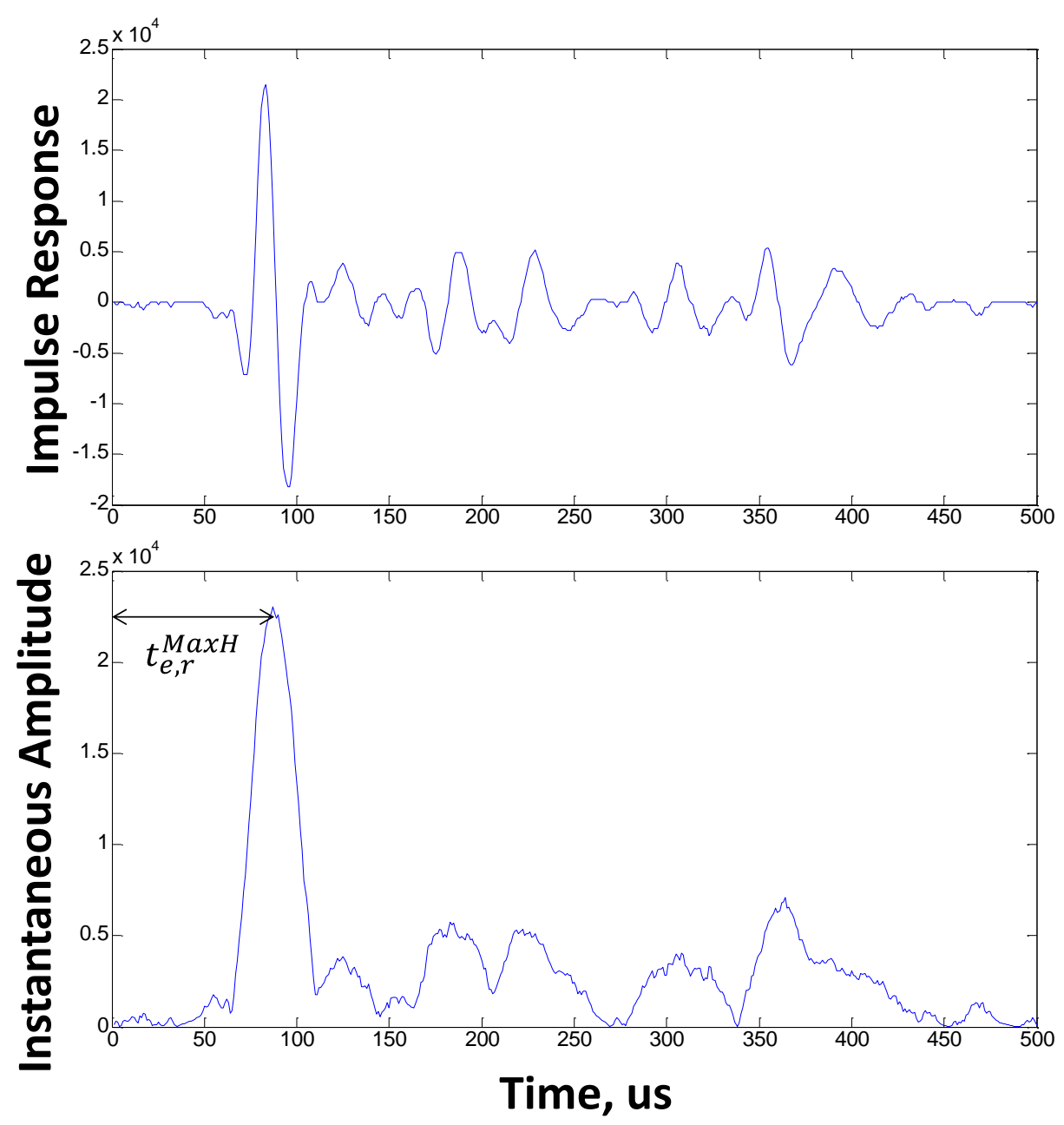

Fig. A.6. Determination of the direct arrival peak using the instantaneous amplitude envelope.

The full-waveform impulse responses are corrected by setting $t=t-t^{S H I F T, H i l b}$ for each sending and receiving pair before it is applied to the SAFT-IA B-scan reconstruction.

Similarly, for the ultrasonic linear array SAFT reconstructions based on the Hilbert transform, we are interested focusing the instantaneous amplitude within the ROI to changes in acoustic impedance. Therefore, the Hilbert transform is performed for each horizontal coordinate SAFT reconstruction in a similar manner to Eq. (A.10) as follows:

$$
\widehat{\boldsymbol{o}}^{I A}(x, z)=\sqrt{(\widehat{\mathrm{o}}(x, z))^{2}+\left(\frac{P}{\pi} \int_{-\infty}^{\infty} \frac{\widehat{o}(x, s)}{z-s} d s\right)^{2}}
$$

where $\widehat{\boldsymbol{o}}^{I A}(x, z)$ defines the instantaneous amplitude-based SAFT reconstruction and $P$ is the principal value of the singular integral in Eq. (A.12). If the ROI is represented by a $W \times D$ set of points located in $W$ columns, equally spaced in the horizontal direction, and $D$ rows, equally spaced in the vertical direction, the matrix formalization in Fig. A.7 can be used to represent the SAFT-IA reconstructed image, $[\widehat{\boldsymbol{O}}]^{I A}$. 


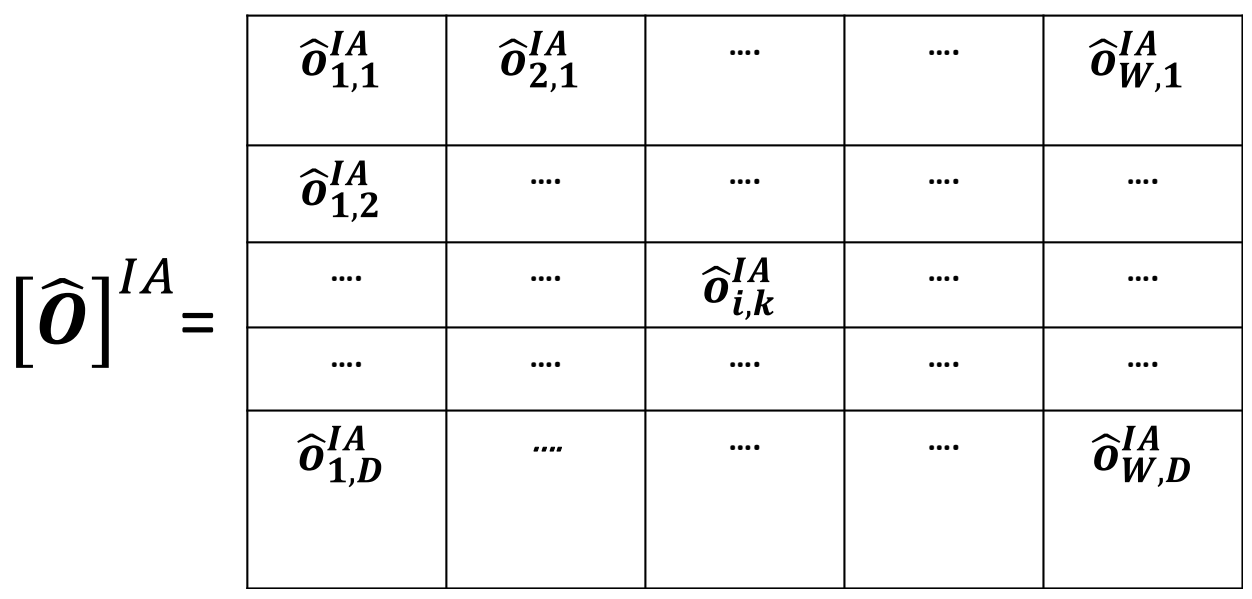
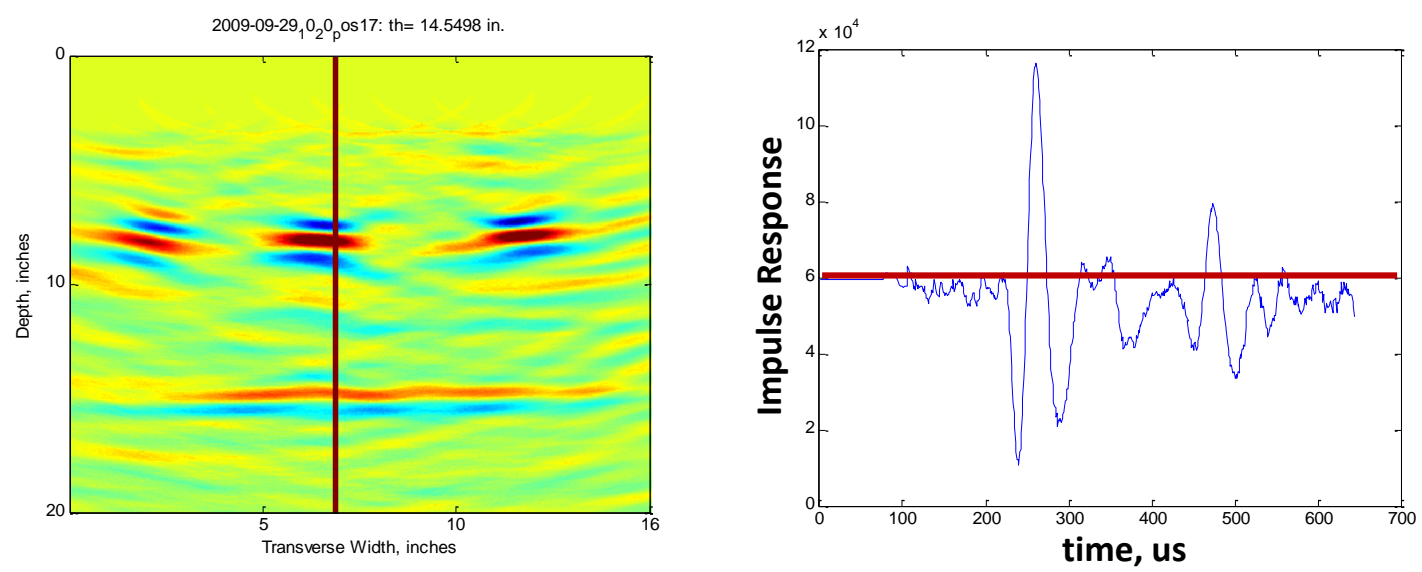

Fig. A.7. SAFT reconstruction and example column data.

Figure A.8 shows the SAFT-IA reconstruction after taking the Hilbert transform given in Eq. (A.12) as well as the column representation of reflectivity along the same edge of the reinforcement, $\hat{o}_{i, k}^{I A}$. It can be observed that the high reflectivity in the SAFT-IA reconstruction better indicates the location of the reinforcement and layer boundary than the original SAFT reconstruction. 

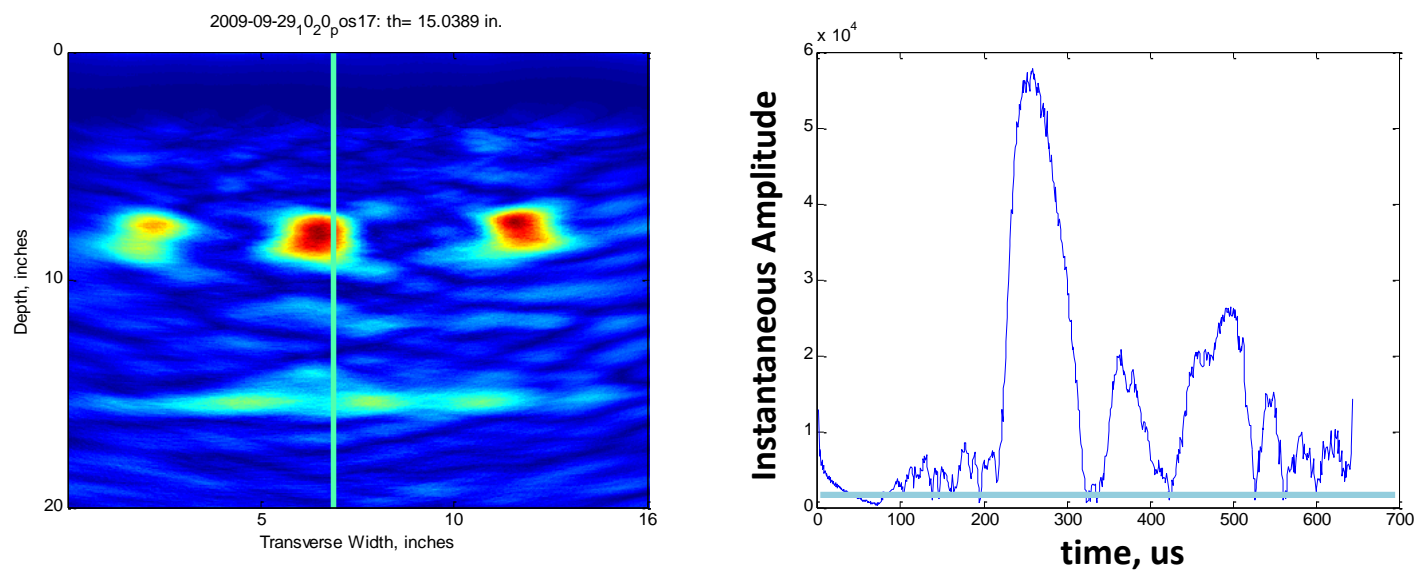

Fig. A.8. SAFT-IA reconstruction and example column data.

Furthermore, the use of the Matlab ${ }^{\mathrm{tm}}$ two-dimensional smoothing function ("disk" filtering) allows for additional elimination of structural noise in the reconstruction. Figure A.9 illustrates the resulting filtered SAFT-IA B-scan of the forensic verification of the reinforcement location. It can be observed that the depth of the reinforcement corresponds to the higher instantaneous amplitude region within the SAFT-IA B-scan reconstruction.

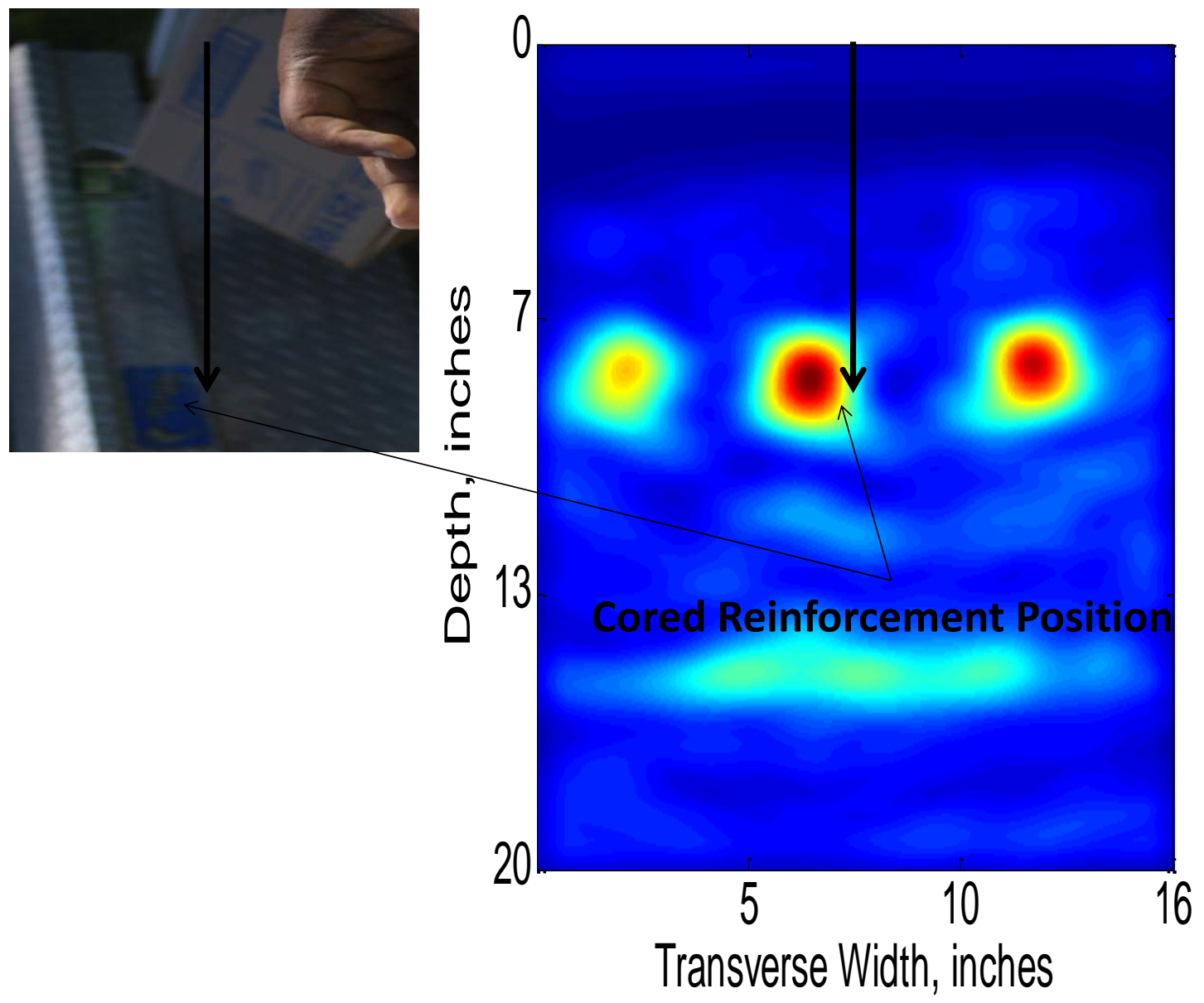

Fig. A.9. Forensic verification of the focused reinforcement location within the SAFT-IA B-scan. 


\section{A.4 SAFT-3D}

Three-dimensional reconstruction of subsurface characteristics can simplify interpretation of multiple ultrasonic array scans. SAFT 3-dimensional reconstructions (SAFT-3D) can be achieved using various interpolation and filtering techniques. Figure A.10 shows the SAFT-IA B-scan from Fig. A.9 as well as 10 additional measurements at $50 \mathrm{~mm}$ step sizes in the longitudinal direction.
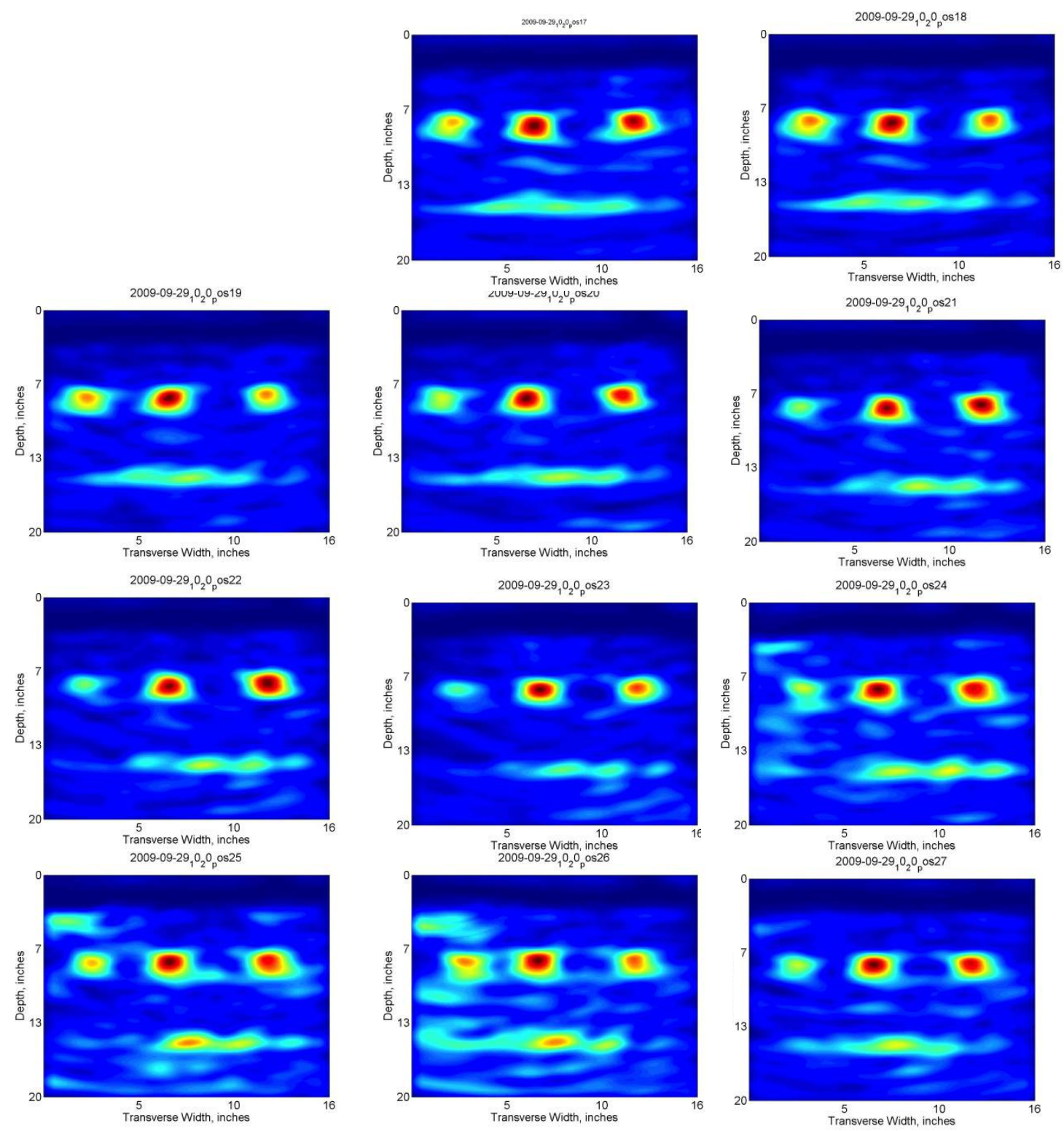

Fig. A.10. SAFT-IA B-scans from the cored location as well as 10 subsequent scans in the longitudinal direction.

These scans were used to create the remaining length of the cored reinforcement as well as the reinforcement to the right of the cored reinforcement in a three-dimensional reconstruction (SAFT-3D). Figure A.11 shows a schematic of the SAFT-3D reconstruction process where scans are taken in step sizes in the longitudinal direction and interpolation techniques are used to create the continuous 3D image of the ROI. The process of creating the SAFT-3D reconstruction included selecting an optimal threshold, using the same "disk" filtering function for each SAFT-IA B-scan, a MATLAB ${ }^{\mathrm{TM}}$ 3D matrix smoothing 
function ("smooth3"), and a MATLAB ${ }^{\mathrm{TM}}$ interpolation function ("isosurface") within the volume of the reconstruction.

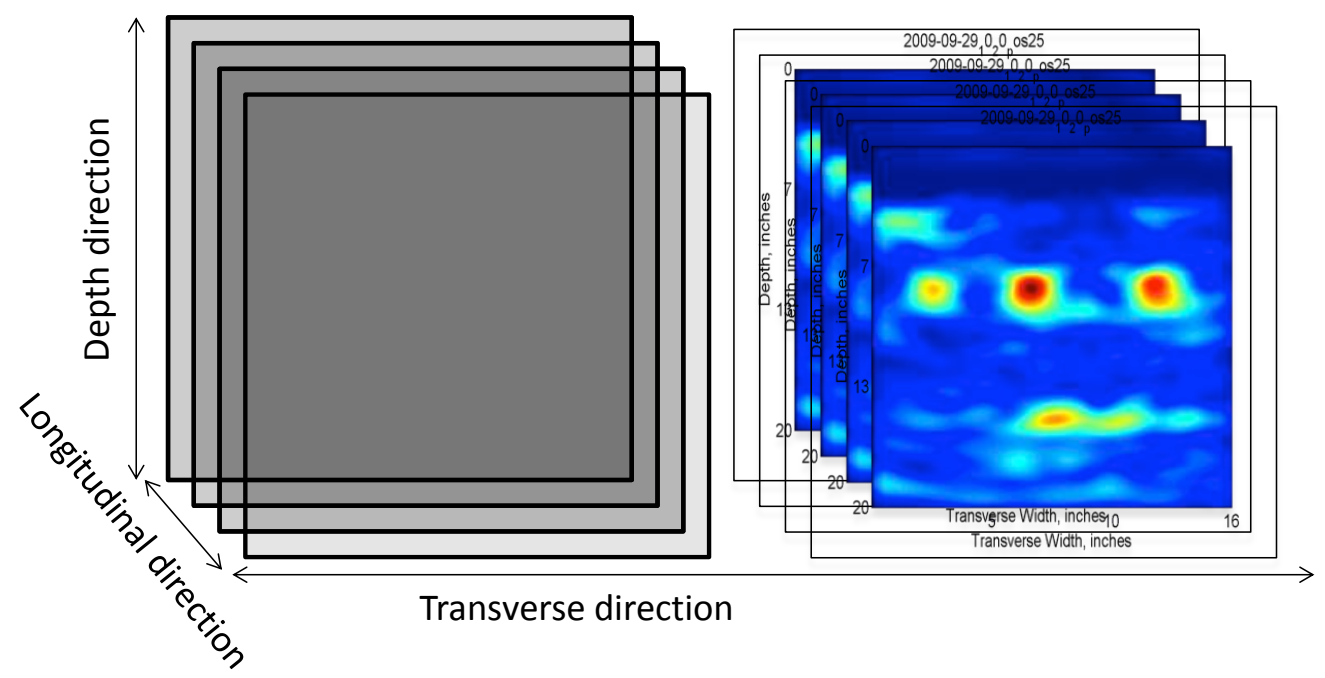

Fig. A.11. Schematic representation of the process of creating SAFT-3D reconstructions.

The SAFT-3D reconstruction of $300 \mathrm{~mm}$ of depth within the concrete layer of a $500 \mathrm{~mm}$ longitudinal $(850 \mathrm{~mm}$ to $1350 \mathrm{~mm}$ ) by $400 \mathrm{~mm}$ transverse location is given in Fig. A.12. The reinforcement on the left side at $850 \mathrm{~mm}$ in the longitudinal direction corresponds to the cored location. This type of SAFT 3D reconstruction can be useful for getting relational information about the high-intensity reflections to determine if the reflection is caused by an as-designed inclusion or damaged concrete.

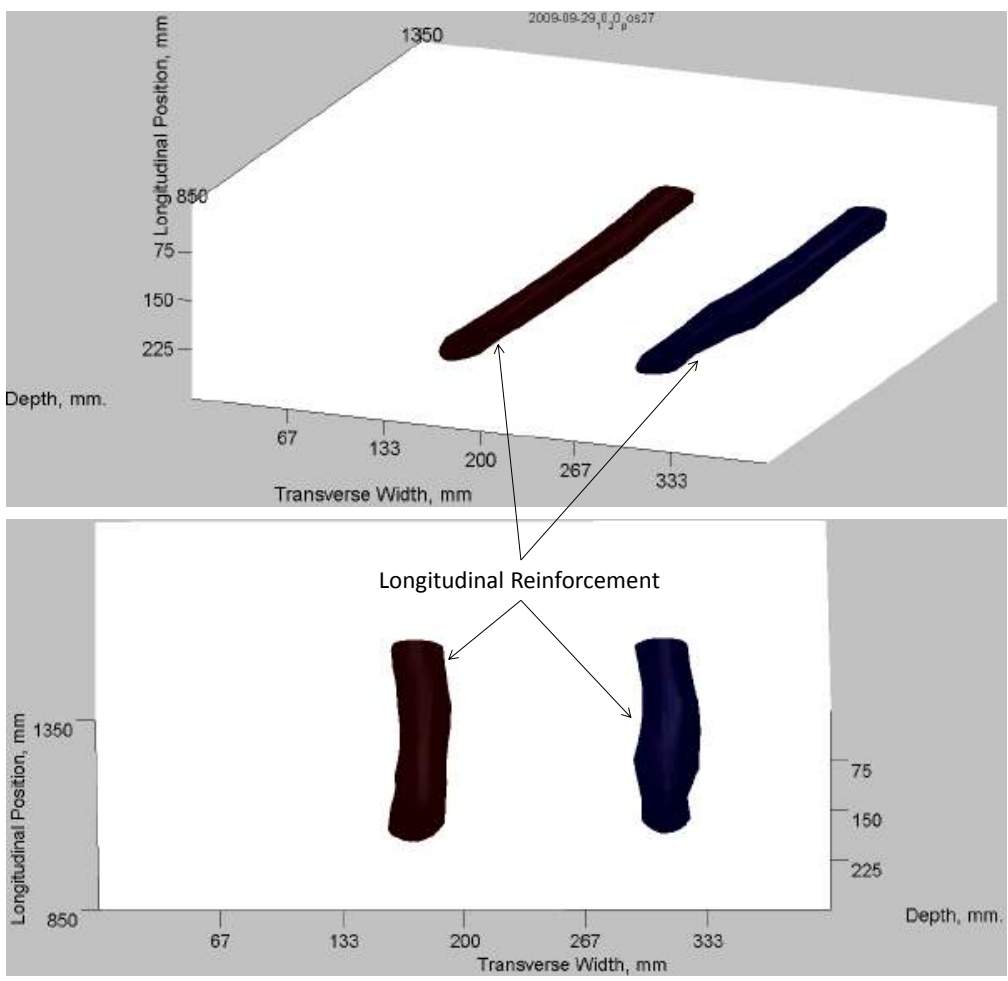

Fig. A.12. SAFT 3D reconstruction using the SAFT-IA B-scan reconstructions shown in Fig. A.10. 


\section{A.5 SAFT PANORAMIC}

While the SAFT and SAFT-IA B-scans are useful for diagnostics of various problems, there are limitations to the use of SAFT B-scans in analysis of some important practical problems. Often the desired ROI is larger than the resulting SAFT B-scan reconstruction from a single set of measurements within the self-contained array allows. As explained by Shokouhi et al. [8], the limited aperture can create situations where "measurements may be inconclusive if the array is located directly above an edge of a defect." To increase the effective aperture and add more redundancy in the measurements, the following procedure was developed to create large panoramic cross sections with increased resolution.

Each SAFT-IA B-scan, $\widehat{\boldsymbol{O}}_{k, i}^{m}$ is converted to the dimensions of the larger region of interest, $R O I_{P A N}$, where $m$ is an index of the current SAFT-IA B-scan. The vertical dimensions of the new $R O I_{P A N}$ and $R O I$ of each SAFT-IA B-scan $\widehat{\boldsymbol{O}}_{k, i}^{m}$ are the same, while the horizontal dimensions are increased. Let the leftmost (lowest) horizontal coordinates of the $m$-th SAFT-IA B-scan, $x_{1}$, correspond to the $x_{i^{*}}$ coordinate within the panoramic reconstruction. In this case all intensity values of the original SAFT-IA B-scan within the new $R O I_{P A N}$ are placed according to their physical location with zeros padding any location where no intensity value applies. Each padded SAFT B-scan, $\widehat{\boldsymbol{O}}_{k, i}^{P A N, m}$, is created according to the following relationship for all $\widehat{\boldsymbol{O}}_{k, i}^{m}$ horizontal positions within the $R O I_{P A N}$ :

$$
\widehat{\boldsymbol{O}}_{k, i}^{P A N, m}=\widehat{\boldsymbol{O}}_{k, i-i^{*}+1}^{m} \text { for } i^{*} \leq i \leq i^{*}+W-1,
$$

and

$$
\hat{O}_{k, i}^{P A N, m}=0 \text { for } i<i^{*} \text { or } i \geq i^{*}+W .
$$

Equation (A.15) shows the matrix representation of an example panoramic form, $[\widehat{\boldsymbol{O}}]^{P A N, m}$, of the $m$-th

\begin{tabular}{|c|c|c|c|c|c|c|c|}
\hline \multirow{4}{*}[\widehat{\boldsymbol{0}}]{$^{P A N, m}=$} & 0 & 0 & $\widehat{\boldsymbol{o}}_{i^{*}, 1}^{P A N, M}$ & .... & $\int_{i^{*}+W-1,1}^{P A N, M}$ & 0 & 0 \\
\hline & 0 & 0 & .... & $\widehat{\boldsymbol{o}}_{i, k}^{P A N, M}$ & .... & 0 & 0 \\
\hline & 0 & 0 & $\ldots$. & $\ldots$ & $\ldots$ & 0 & $\mathbf{0}$ \\
\hline & 0 & 0 & $\widehat{\boldsymbol{o}}_{\boldsymbol{i}^{*}, \boldsymbol{D}}^{P A N, M}$ & .... & $\widehat{\boldsymbol{o}}_{W, D}^{P A N, M}$ & 0 & $\mathbf{0}$ \\
\hline
\end{tabular}
applied SAFT-IA B-scan.

After this procedure, the individual scans can be combined into a single panoramic representation, SAFT-Pan, using the following rule: $\widehat{\boldsymbol{O}}_{k, i}^{P A N}={ }_{m}^{\max }\left(\widehat{\boldsymbol{\boldsymbol { O }}}_{k, i}^{E P A N, m}\right)$ for all $k$ and $i$ in the $\mathrm{ROI}_{P A N}$, where $[\widehat{\boldsymbol{O}}]^{P A N}$ is the matrix form of the SAFT-Pan.

To illustrate the use of the SAFT-IA Panoramic method (SAFT-Pan), a series of scans at a Portland Cement Concrete (PCC) joint with embedded dowels is presented. The SAFT-Pan reconstructions show high levels of reflection intensity at lateral and depth locations where there is a change in acoustic impedance such as a dowel inclusion or at the interface between the concrete and base material. Figure A.13 shows nine SAFT-IA B-scans each centred approximately 5 in. from a transverse joint with 12 in. thickness and 1.5 in. diameter dowels inserted at the mid-depth of the pavement. It can be observed that 
round high-intensity reflections (red) are located at about half the depth of the more oblong high-intensity reflection (red) at a greater depth.

The round reflections indicate the lateral location and depth of the dowels, while the oblong reflection indicates the depth of the PCC pavement layer. It can be observed that locations other than the doweled locations or PCC depth have a low intensity of reflection, indicating low reflectivity (sound) surrounding concrete.

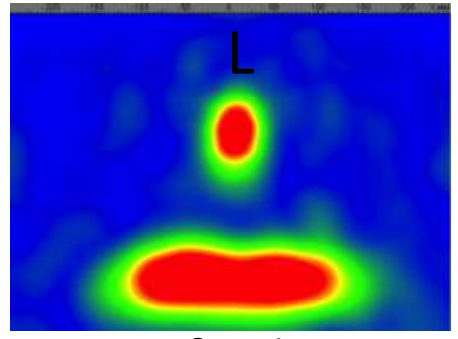

Scan 1

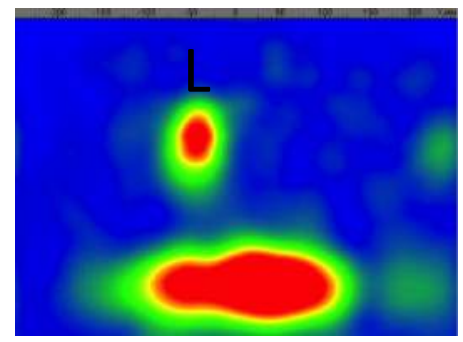

Scan 2

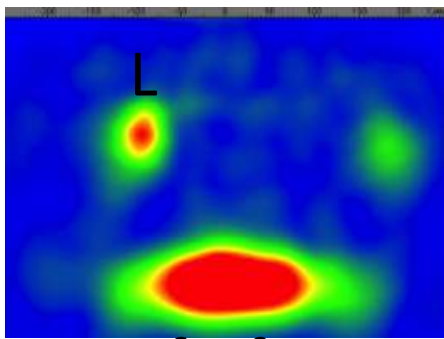

Scan 3

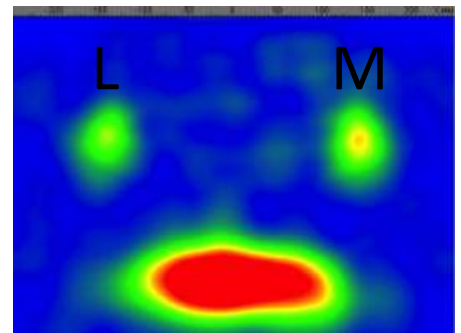

Scan 4

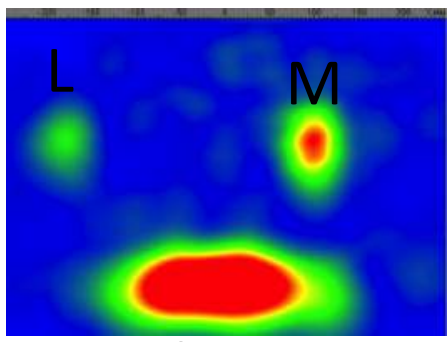

Scan 5

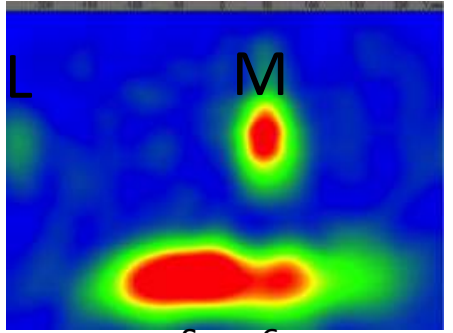

Scan 6

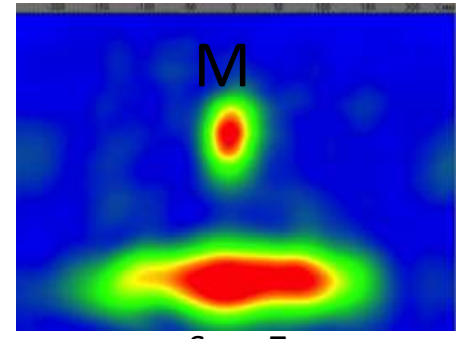

Scan 7

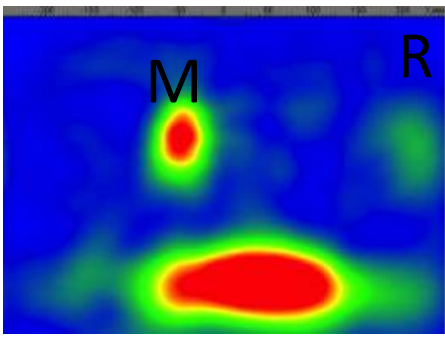

Scan 8

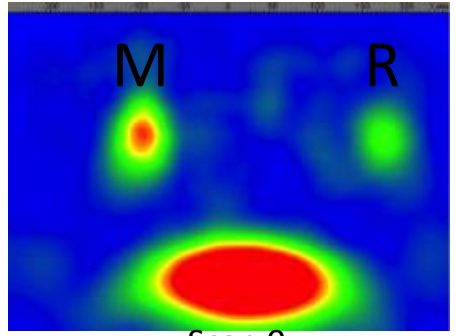

Scan 9

Fig. A.13. Example set of nine overlapping SAFT-IA B-scans used to create a SAFT Panoramic.

Figure A.14 shows the SAFT-Pan reconstruction resulting from the nine overlapping SAFT-IA B-scans taken in $5.08 \mathrm{~cm}$ step sizes after they have been fused together. The resulting panoramic tomography indicates the subsurface condition of a $3 \mathrm{ft}$ wide section of the pavement. Analysis of Fig. A.14 indicates a low reflectivity (relatively sound) condition where the only high intensity of reflection occurs due to features that were as designed including the slightly less than $1 \mathrm{ft}$ concrete depth reflection and circular reflections at the depth and lateral location of the dowels. The lack of reflection (blue) at the remaining locations indicates undamaged concrete. This illustrates that SAFT-Pan reconstructions can create a clearer picture of inclusions and their relative positions than individual SAFT-IA reconstructions. SAFT-Pan reconstructions can be used to create SAFT-3D Reconstructions using the same process described in Sect. A.4. 


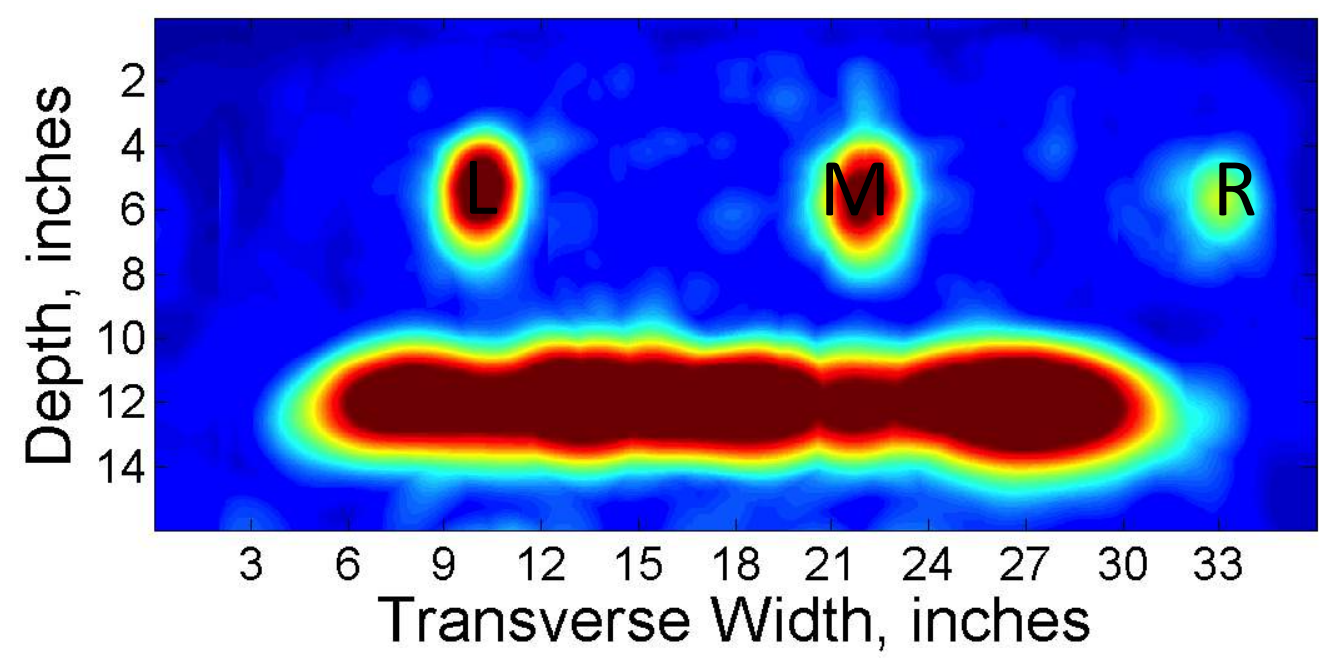

Fig. A.14. Five SAFT-Pan examples at a PCC joint.

\section{A.6 AUTOMATED DATA INTERPRETATION FOR CONCRETE}

While the Kirchoff-migration-based SAFT reflectivity reconstructions discussed in Section A.1 create an intuitive focused image of subsurface reflectivity in pavements, qualitative analysis methods require expertise and can be time-consuming. Quantitative methods can be useful to create automated analysis of specific pavement-related problems to increase productivity while often improving the accuracy and reliability of the interpretation.

\section{A.7 DIRECT REFLECTION AUTOMATION}

The method used for direct reflection quantification should be capable of identifying the characteristics of the object of interest (e.g., reinforcement location, pavement thickness interface, delamination, etc.). This can be done with the following general algorithm.

- Run the applicable SAFT analysis described in Sect. A.1 to estimate the reflectivity function of the region

- Identify threshold value that will separate high intensity of reflection areas from low intensity of reflection areas

- Identify characteristics of the type of reflection caused by the specific object of interest

- Use shape recognition schemes to decide if the identified areas are in fact the object of interest based on the identified characteristics

- Determine the location (depth and lateral position) of the center of the object of interest

- Eliminate false positives through a check with one or multiple adjacent scans

- Output the results to a spreadsheet along with information about the scan locations

The general outline given above was used to develop an algorithm capable of finding depth of reinforcements or any round inclusion in concrete pavements. Figure A.15 shows the steps (left top to 
bottom, then right top to bottom) in identifying two longitudinal rebar elements in an example SAFT Bscan similar to the example described in Sect. A.1. The figure illustrates the use of circularity criteria to identify longitudinal rebar reflections. After a threshold value of 0.80 of the maximum intensity is applied, the two central reflections are identified as reinforcement, while the left and right reflections are rejected because their characteristics could not be determined reliably. Thus, in this case, the concrete cover for the identified center two reflections would be output to the spreadsheet.
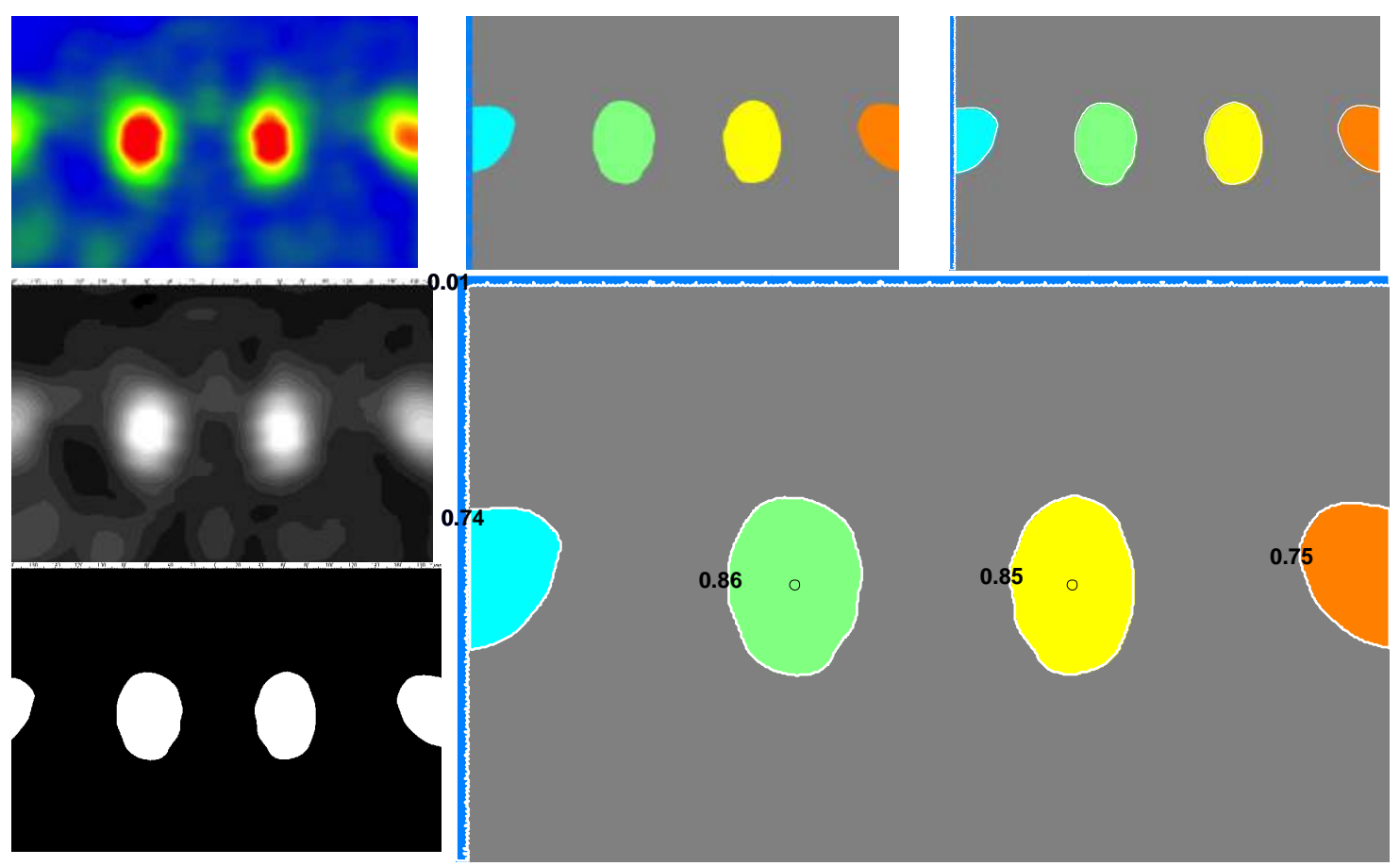

Fig. A.15. Progression in identifying the centroid of reflections caused by round inclusions.

The same general procedure was used to develop an algorithm for automated layer boundary depth detection. In this case, the depth of the reflecting interface was determined by choosing the highest average reflectivity of the rows in the reconstruction. The horizontal portion to be included within each row can be predefined based on the characteristics of the layer boundary.

\section{A.8 FLAW DETECTION}

As discussed in Sect. A.7, identification of inclusions and layer boundaries in concrete from SAFT reconstructions can be automated using shape recognition and absolute maxima techniques. These methods were feasible because the geometry of the area of interest was known within each scan and in relation to adjacent scans. However, flaws in concrete such as improper concrete consolidation are generally nonuniform and a priori knowledge of the reflector type and dimension is not available. Automated detection of these types of defects, which are stochastic in nature, requires a method that does not rely on shape recognition.

To accomplish this task, the impact-echo signature analysis (IESA) method [9] was modified for the reconstruction methods used in this study. While Schubert and Koehler stated that a priori knowledge of a reference signal where no scatterers are present is not generally available, the Kirchoff migration methods described in Sect. A.1 provide intuitive reference scans. It is proposed to generalize the IESA method for use with SAFT reconstructions that are obtained from the ultrasonic linear array or other tomography reconstructions. 
This can be accomplished by generalizing the IESA into a two-dimensional ultrasonic tomography signature analysis method (2D-UTSA or UTSA). Pearson's correlation coefficient is adapted for comparison of reconstructed intensity matrices as follows using the SAFT-IA B-scan variables introduced in Sect. A.1.2:

$$
C^{I A, m}=\frac{\operatorname{cov}\left[[\hat{o}]^{I A, r e f},[\hat{o}]^{I A, m}\right]}{\sqrt{\operatorname{Var}\left[[\hat{o}]^{I A, r e f}\right] \operatorname{Var}\left[[\hat{o}]^{I A, m}\right]}}=\frac{\sum_{i=1}^{W} \sum_{k=1}^{D}\left(\hat{o}_{i, k}^{I A, r e f}-\hat{o}_{\text {mean }}^{I A, r e f}\right)\left(\hat{o}_{i, k}^{I A, m}-\hat{o}_{\text {mean }}^{I A, m}\right)}{\sum_{i=1}^{W} \sum_{k=1}^{D}\left(\hat{o}_{i, k}^{I A, r e f}-\hat{o}_{\text {mean }}^{I A, r e f}\right)^{2} \sum_{i=1}^{W} \sum_{k=1}^{D}\left(\hat{o}_{i, k}^{I A}-\hat{o}_{\text {mean }}^{I A}\right)^{2}},
$$

where $[\widehat{\boldsymbol{O}}]^{\text {IAref }}$ and $[\widehat{\boldsymbol{O}}]^{I A, m}$ are the matrices of reflection intensity for the reference SAFT-IA B-scan and $m$-th SAFT-IA B-scan, respectively; $\widehat{\boldsymbol{o}}_{i, k}^{I A, r e f}$ and $\widehat{\boldsymbol{o}}_{i, k}^{I A}$ are the single intensity values of the reference signal and $m$-th reconstruction, respectively, with depth below the measurement location increasing with $k$ and the location along the horizontal direction of the scan increasing with $i$; $\widehat{\boldsymbol{o}}_{\text {mean }}^{I A \text {,ref }}$ and $\widehat{\boldsymbol{o}}_{\text {mean }}^{I A}$ are the mean intensities of the reference scan and $m$-th scan, respectively; $W$ and $D$ are the number of width and depth intensity values in the depth and device aperture direction, respectively; and $C^{I A, m}$ is Pearson's correlation coefficient, which measures the strength of the linear dependence between $[\widehat{\boldsymbol{o}}]^{\text {IAref }}$ and $[\widehat{\boldsymbol{O}}]^{I A, m}$.

Thus, if a SAFT-IA B-scan taken on relatively sound concrete with similar structural geometry is used as the reference scan, flawed concrete locations can be identified. On the extremes, a $C^{I A, m}$ value of 0 would indicate no correlation and a $C^{I A, m}$ value of 1 would indicate that the two SAFT-IA B-scans are related linearly. Therefore, a higher $C^{I A, m}$ would indicate that the m-th SAFF-IA B-scan was taken on sound concrete, and a significant decrease in the correlation coefficient would indicate non-uniform SAFT-IA B-scans, or flawed concrete, especially if observed in a group of adjacent scans. This type of analysis will be referred to as the 2D-UTSA method.

In addition to making the method applicable for the type of data gathered by the study, the use of the expanded Pearson's correlation for 2D comparison improves the method by correcting the issues of the IESA technique in selecting the reference signal. As explained in Sect. A.1.2, the SAFT-IA reconstruction creates a relatively intuitive reconstructed image of the ROI reflectivity function. Therefore, selection of a damage-free reference scan is possible based on past experience with signal interpretation of SAFT reconstructions [10-13], and the misinterpretations associated with the IESA method can be mitigated. When necessary, coring should be conducted to verify that the reference scan is indeed damage free.

Furthermore, subsurface damage in concrete is generally entropic in that there is little variation between SAFT-IA B-scans of concrete in relatively good condition at different locations if the same instrument settings are used, while there is a significant variation between scans where flaws are present at different locations. Therefore, sound concrete will have similar levels of correlation with the reference SAFT-IA B-scan, whereas the correlation of scans with flaws at different locations will fluctuate. Therefore, a procedure where the reference scan is taken as the average of all of the SAFT-IA B-scans in the set is introduced. It is expected that sound concrete may not necessarily have as high a correlation with the generated reference scan as is the case for a manually selected reference scan. However, if a significant portion of measurements are made on sound concrete, the sound concrete locations should result in similar correlation values, while unsound concrete will result in lower values due to the randomness of flaws. Thus, decreases are still present in the correlogram even when the reference scan includes contributions from the flawed concrete locations. This method is not overly sensitive to selection of the reference scan and can be generally applied to locate areas of flawed concrete. 


\section{A.9 SAFT PANORAMIC-ENHANCED}

While SAFT-Panoramic analysis is useful for many applications, there are some situations where the physical location of each SAFT-IA B-scan is not known to the desired accuracy of the reconstruction. In this case, the SAFT-Pan procedure described in Sect. A.5 can introduce significant error. Figure A.16 shows an example of a SAFT-Pan reconstruction which combines nine individual SAFT-IA reconstructions. The target step size of 2 in. was used in SAFT-Pan development, which resulted in a blurred reflectivity in the region of the center dowel due to imprecise step size inputs.

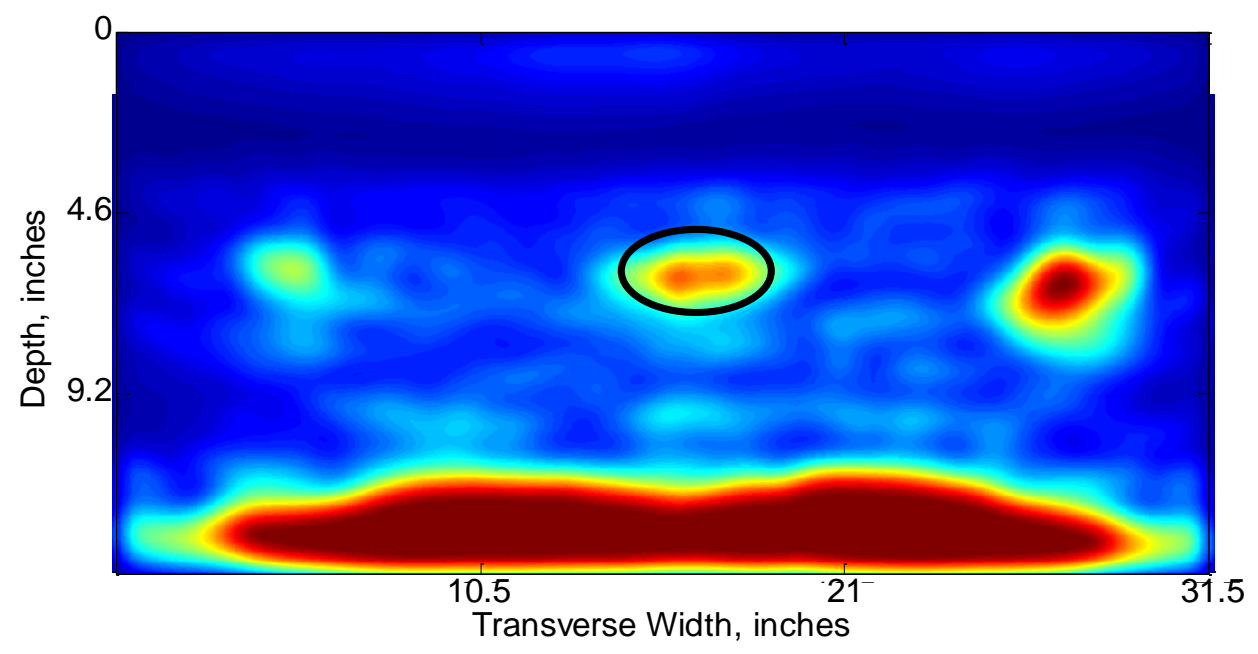

Fig. A.16. SAFT-Pan reconstruction with imprecise step size input.

To address this problem, an iterative procedure for enhanced panoramic reconstruction was developed. First, each SAFT-IA B-scan is numbered according to increasing coordinate in the horizontal direction. Then, the individual SAFT-IA B-scans are added sequentially. The procedure is based on the concept that the same position within the ROI should result in similar relative reflectivity, regardless of the location of the scan, assuming the effect of limited aperture is taken into account.

Denote $[\widehat{\boldsymbol{O}}]^{E P A N, m}$ as the panoramic reconstruction after the $m$-th SAFT-IA B-scan, $[\widehat{\boldsymbol{O}}]^{I A, m}$, is added and $W^{E P A N, m}$ is the number of columns. Naturally, $[\widehat{\boldsymbol{O}}]^{E P A N, 1}=[\widehat{\boldsymbol{O}}]^{I A, 1}$ and $W^{E P A N, 1}=W$, where, as defined earlier, $W$ is the number of columns in the individual SAFT-IA B-scan reconstructions. Unlike Sect. A.1.4, the exact difference in positions of adjacent positions, $\Delta S$, is not known but is assumed to be within a certain range defined by Eq. (A.17):

$$
\iota_{1} \Delta x \leq \Delta S \leq \iota_{2} \Delta x
$$

where $\iota_{1}$ and $\iota_{2}$ are integers and $\Delta x$ is the difference in horizontal position between two adjacent columns in the reconstruction. This means that the difference in adjacent positions can be expressed in terms of the number of additional columns, $\iota$. For each $\iota$ within the range $\left[\iota_{1}, l_{2}\right]$, a similarity between portions of the overlapping regions within $[\widehat{\boldsymbol{O}}]^{E P A N, m}$ and $[\widehat{\boldsymbol{O}}]^{I A, m+1}$ is determined. To account for the effect of limited aperture, the first $W_{1}$ columns of $[\widehat{\boldsymbol{O}}]^{I A, m+1}$ and last $W_{1}$ columns of $[\widehat{\boldsymbol{O}}]^{E P A N, m}$ are not considered in determining similarity. In many cases, the stability of the process is improved if only a portion of the reconstruction in the vertical direction $\left[D_{1}, D_{2}\right]$ is included in the comparison. Figure A.17 illustrates the process of determining overlapping regions used in the similarity analysis. 


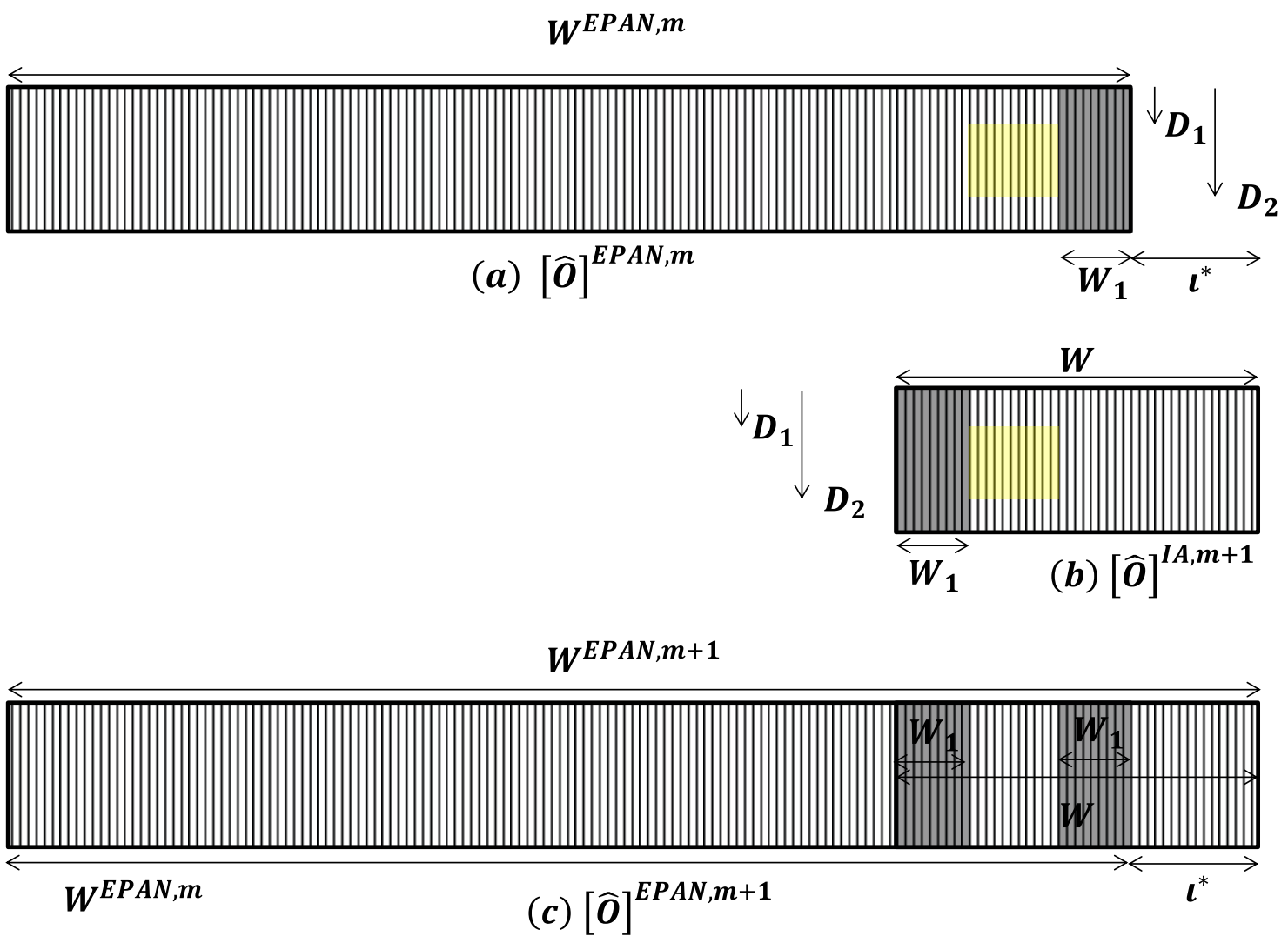

Fig. A.17. Determination of overlapping regions between the reconstructions and determination of the next panoramic reconstruction.

The degree of similarity of the overlapping regions, $H(\iota)$, is determined based on Pearson's Correlation as follows:

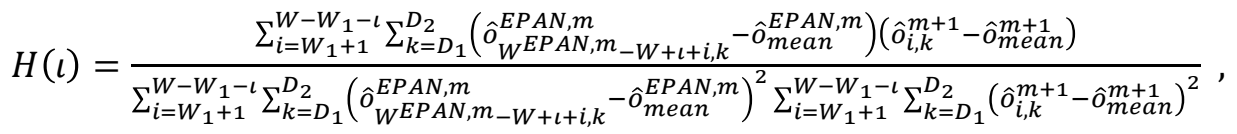

where

$$
\hat{\mathrm{o}}_{\text {mean }}^{\mathrm{EPAN}, \mathrm{m}}=\frac{\sum_{\mathrm{i}=\mathrm{W}^{\mathrm{EPAN}, \mathrm{m}}-\mathrm{W}+\mathrm{l}+\mathrm{W}_{1}+1}^{\mathrm{W}_{\mathrm{EPAN}, \mathrm{m}}^{\mathrm{EP}} \sum_{\mathrm{k}=\mathrm{D}_{1}}^{\mathrm{D}_{2}} \hat{\mathrm{o}}_{\mathrm{i}, \mathrm{k}}^{\mathrm{EPAN}, \mathrm{m}}}}{\left(\mathrm{W}-2 \mathrm{~W}_{1}-\mathrm{l}\right)\left(\mathrm{D}_{2}-\mathrm{D}_{1}+1\right)}
$$

and

$$
\hat{\mathrm{o}}_{\text {mean }}^{\mathrm{m}+1}=\frac{\sum_{\mathrm{i}=\mathrm{W}_{1}+1}^{\mathrm{W}-\mathrm{W}_{1}-\mathrm{l}} \sum_{\mathrm{k}=\mathrm{D}_{1}}^{\mathrm{D}_{2}} \hat{\mathrm{o}}_{\mathrm{i}, \mathrm{k}}^{\mathrm{m}+1}}{\left(\mathrm{~W}-2 \mathrm{~W}_{1}-\mathrm{l}\right)\left(\mathrm{D}_{2}-\mathrm{D}_{1}+1\right)} .
$$

The $\iota$ that results in the maximum value of function $H(\iota)$ on the interval $\left[\iota_{1}, \iota_{2}\right]$ is denoted as the optimal shift factor, $\iota^{*}$. Then the next SAFT-EPAN reconstruction, $[\hat{O}]^{E P A N, m+1}$, is defined as follows: 


$$
\begin{gathered}
\hat{O}_{i, k}^{E P A N, m+1}=\widehat{O}_{i, k}^{E P A N, m} \text { for } i<W^{E P A N, m}-W+\iota^{*}+W_{1}, \\
\widehat{\mathrm{O}}_{\mathrm{i}, \mathrm{k}}^{\mathrm{EPAN}, \mathrm{m}+1}=\max \left(\widehat{\mathrm{O}}_{\mathrm{i}, \mathrm{k}}^{\mathrm{EPAN}, \mathrm{m}}, \widehat{\mathrm{O}}_{\mathrm{i}-\mathrm{W}^{\mathrm{EPAN}, \mathrm{m}}+\mathrm{W}-\iota^{*}, \mathrm{k}}^{\mathrm{m}+1}\right),
\end{gathered}
$$

for

$$
W^{E P A N, m}-W+\iota^{*}+W_{1} \leq i \leq W^{E P A N, m},
$$

and

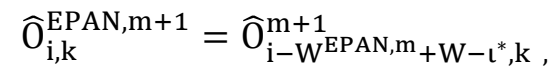

for

$$
W^{E P A N, m}<i \leq W^{E P A N, m+1}
$$

where

$$
W^{E P A N, m+1}
$$

is the width of the new SAFT-EPAN as defined by

$$
W^{E P A N, m+1}=W^{E P A N, m}+\iota^{*} .
$$

Each subsequent SAFT panoramic reconstruction, $[\widehat{\boldsymbol{O}}]^{E P A N, m+1}$, is obtained from addition of $[\widehat{\boldsymbol{O}}]^{E P A N, m}$ and the next SAFT-IA B-scan, $[\widehat{\boldsymbol{O}}]^{I A, m+1}$. The additional number of columns in the new reconstruction, $\iota^{*}$, is determined by comparing the similarity between portions of the overlapping regions within $[\widehat{\boldsymbol{O}}]^{E P A N, m}$ and $[\widehat{\boldsymbol{O}}]^{I A, m+1}$. The additional columns, $\iota^{*}$, are chosen from within the range of potential shift factors $\left[\iota_{1}, \iota_{2}\right]$.

Figure A.18 illustrates the similarity of overlapping scans as a function of possible shift factors, $H(\iota)$, used for fusing of the nine scans from this example. The plot is given on an $x$-axis scale where columns are converted to inches and the target shift factor of $2 \mathrm{in}$. is marked by the black vertical line. The column range, $\left[\iota_{1}, \iota_{2}\right]$, used for determination of the optimal shift factor, $\iota^{*}$, is equivalent to a range of $1.4 \mathrm{in}$. to 2.6 in. It can be observed that the optimal step sizes, as determined by the $\iota$ resulting in the peak of the $H(\iota)$ curves, are significantly different from the target shift factor at various locations. Smaller-than-target shift factors, $\iota^{*}$, were used for placement of $m=2,4,6,8$ SAFT-IA scans, $[\widehat{\boldsymbol{O}}]^{I A, m}$, while larger-thantarget shift factors, $\iota^{*}$, were used for placement of $m=3,5,7,9$ SAFT-IA scans, $[\widehat{\boldsymbol{O}}]^{I A, m}$ into each iterative formulation of the SAFT-Epan, $[\widehat{\boldsymbol{O}}]^{E P A N, m}$. 

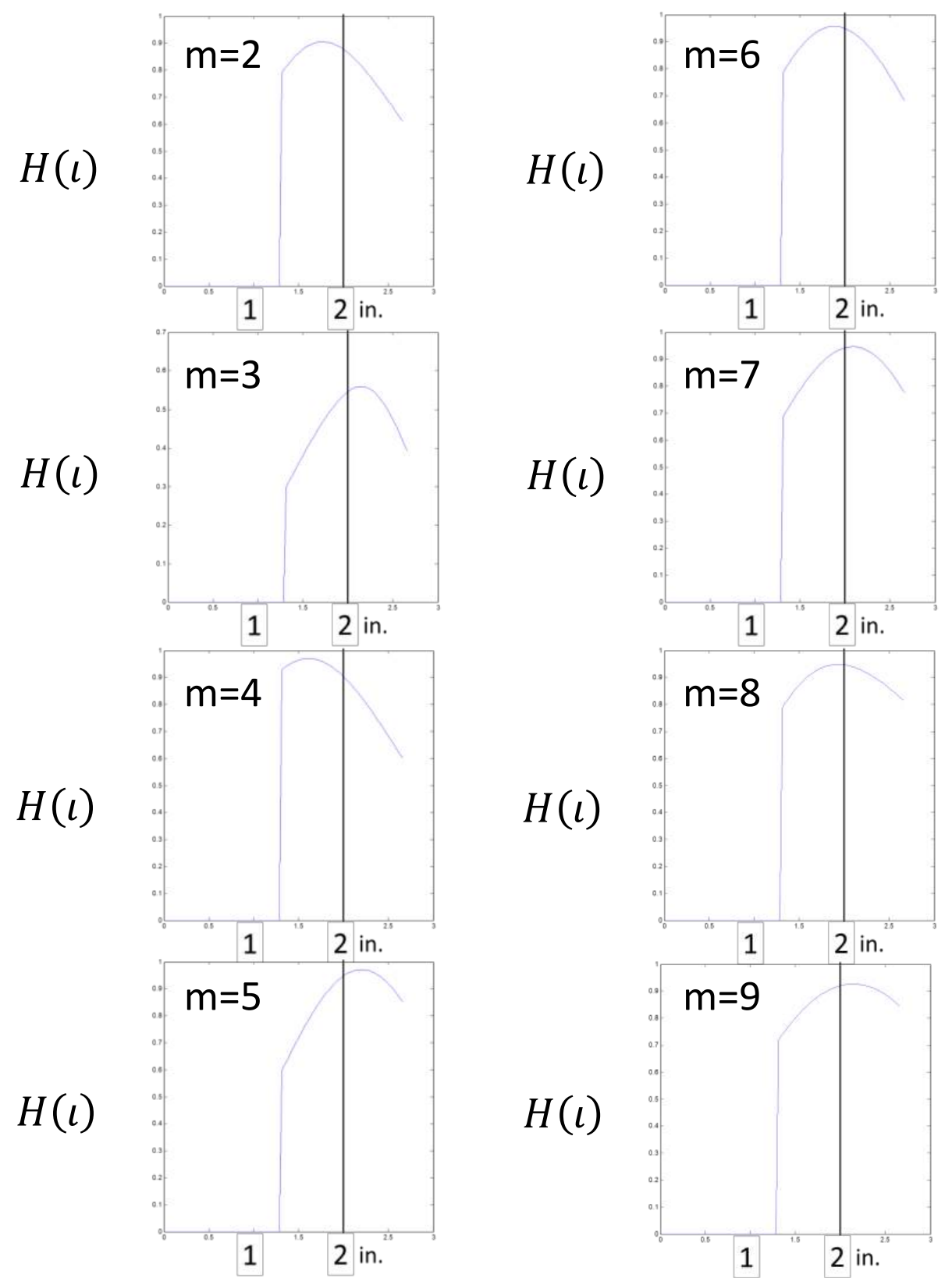

Fig. A.18. Similarity of overlapping region curves used for placement of SAFT-IA reconstructions into the SAFT-EPan reconstruction.

Figure A.19 shows the (a) original SAFT-Pan reconstruction and (b) SAFT-EPan reconstruction both obtained from the same nine individual SAFT-IA scans in this example. It can be observed that the blurry oblong reflection at the center dowel in the SAFT-Pan is a more focused circular reflection when using the SAFT-EPan reconstruction. Correcting for some of the uncertainty in the measurement process by placing the scans based on similarity of overlapping regions allows for a more focused reconstruction that is consistent with the reflectivity in the region. 


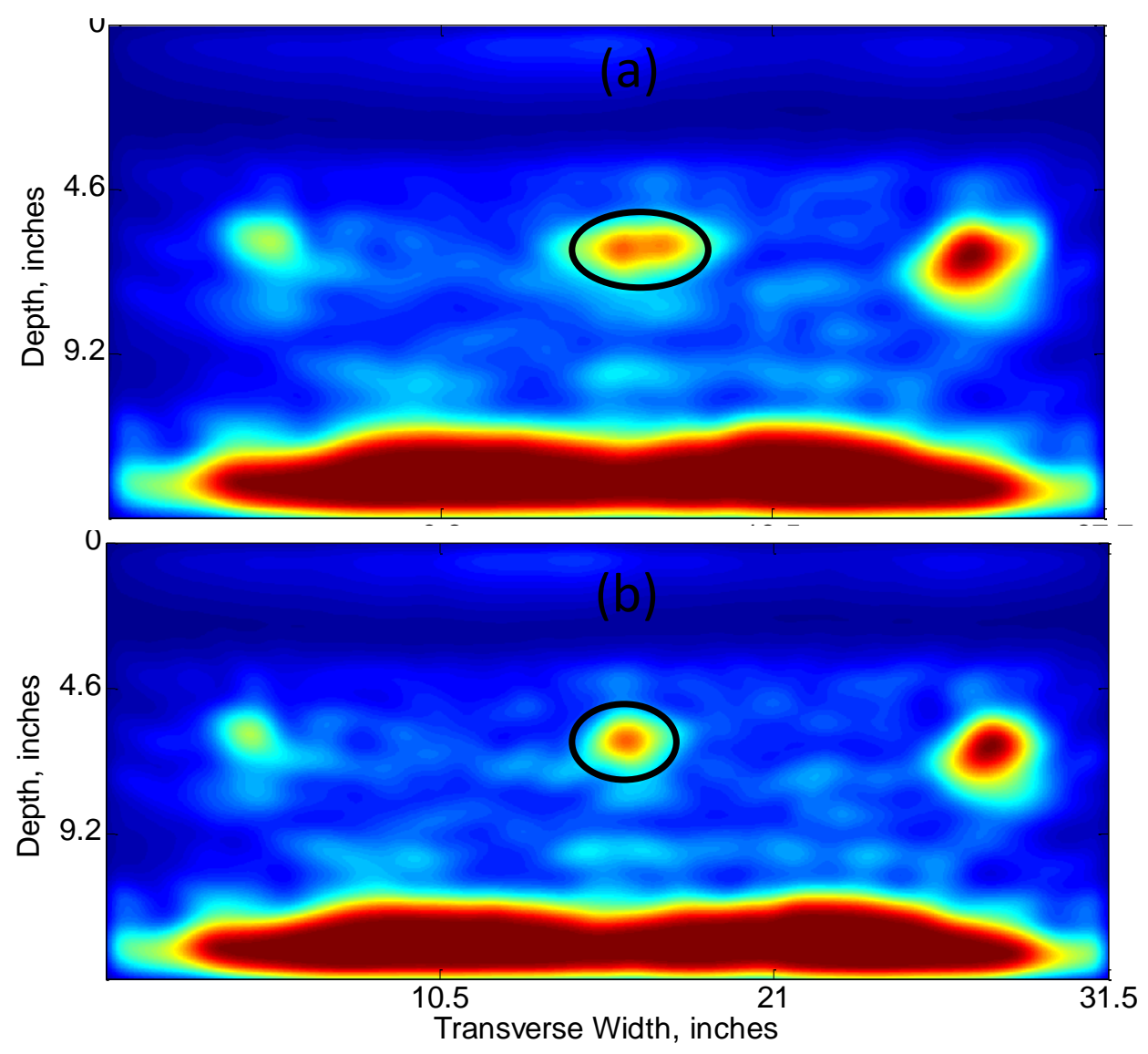

Fig. A.19. Reconstruction of nine overlapping scans over three dowels to create (a) SAFT-Pan and (b) SAFT-EPan reconstructions. 


\section{APPENDIX A REFERENCES}

1. S. L. Hahn, Hilbert Transforms in Signal Processing, Artech House, Boston, 1996.

2. N. E. Huang and N. O. Attoh-Okine, The Hilbert-Huang Transform in Engineering. CRC, 2005.

3. F. R. Kschischang, The Hilbert Transform. University of Toronto, 2006.

4. G. Todoran, R. Holonec, and C. Iakab, Discrete Hilbert Transform. Numeric Algorithms, 2008.

5. L. Marple Jr., "Computing the Discrete-time 'Analytic' Signal via FFT," IEEE Transactions on Signal Processing, 47, 2600-2603 (1999).

6. B. Gold, A. V. Oppenheim, and C. M. Rader, "Theory and Implementation of the Discrete Hilbert Transform," Proceedings of the Symposium on Computer Processing in Communications, New York, 1970.

7. A. V. Oppenheim, R. W. Schafer, and J. R. Buck, Discrete-time Signal Processing, Prentice Hall, Englewood Cliffs, N.J., 1989.

8. P. Shokouhi, J. Wostmann, G. Schneider, B.Milmann, A. Taffe, and H. Wiggenhauser, "Nondestructive Detection of Delamination in Concrete Slabs", in Transportation Research Record: Journal of the Transportation Research Board, 2251(1), 103-113 (2011).

9.

10. R. Schubert and B. Koehler, "Three-dimensional Time Domain Modeling of Ultrasonic Wave Propagation in Concrete in Explicit Consideration of Aggregates and Porosity," Journal of Computational Acoustics, 9(4), 1543-1560 (2001).

11. K. Hoegh, L. Khazanovich, and H. T. Yu, "Ultrasonice Tomography for Evaluation of Concrete Pavements," Transportation Resarch Record: Journal of the Transportation Research Board, 2232, 85-94 (2011).

12. K. Hoegh and L. Khazanovich, "Correlation Analysis of 2D Tomographic images for Flaw Detection in Pavement," ASTM International Journal of Testing and Evaluation, 40(2) (March 2012).

13. K. Hoegh, L. Khazanovich, and T. Yu, "Concrete Pavement Joint Diagnostics Using Ultrasonic Tomography, Transportation Research Board 91st Annual Meeting, 2012. 

APPENDIX B. COMPLETE DETAILS OF FREQUENCY BAND SELECTION 



\section{APPENDIX B. COMPLETE DETAILS OF FREQUENCY BAND SELECTION}

Once a mother wavelet is chosen, the next decisions involve which specific frequency bands are worth segmenting, reconstructing, and running SAFT on. In Figs. B.2-B.26, all of the frequency bands hypothesized to be worthwhile are displayed. Both the "straight" SAFT results as well as the envelope of the "straight" SAFT results are shown to fully convey the information contained in each frequency band. The envelope is created by taking the absolute value of the Hilbert transform of the "straight" SAFT results. It is clear that some frequency bands are of no value, such as the 0 to $7.8125 \mathrm{kHz}$ band (Node 63) and the 7.8125 to $15.625 \mathrm{kHz}$ band (Node 64). As the frequency bandings in Figs. B.2-B.26 become more and more narrow and focused, the frequency ranges containing the energy of the ultrasonic signals can be more specifically identified. Eventually, the frequency bands can become so narrow that there is no useful information in the band. This can quickly be assessed by examining the numeric range represented by each image's color bar.

Figure B.1 shows a wavelet packet decomposition tree for a single signal taken from the sound concrete with dowel dataset. Each decomposition node is labeled with the node index number and the percentage of the signal's total energy contained in each node. While this is just for a single signal from the 45 signals that make up one MIRA version 1 acquisition, it is representative of all 45 signals and therefore provides a reasonable baseline to use in conjunction with the actual SAFT reconstructions shown in Figs. B.2-B.26.

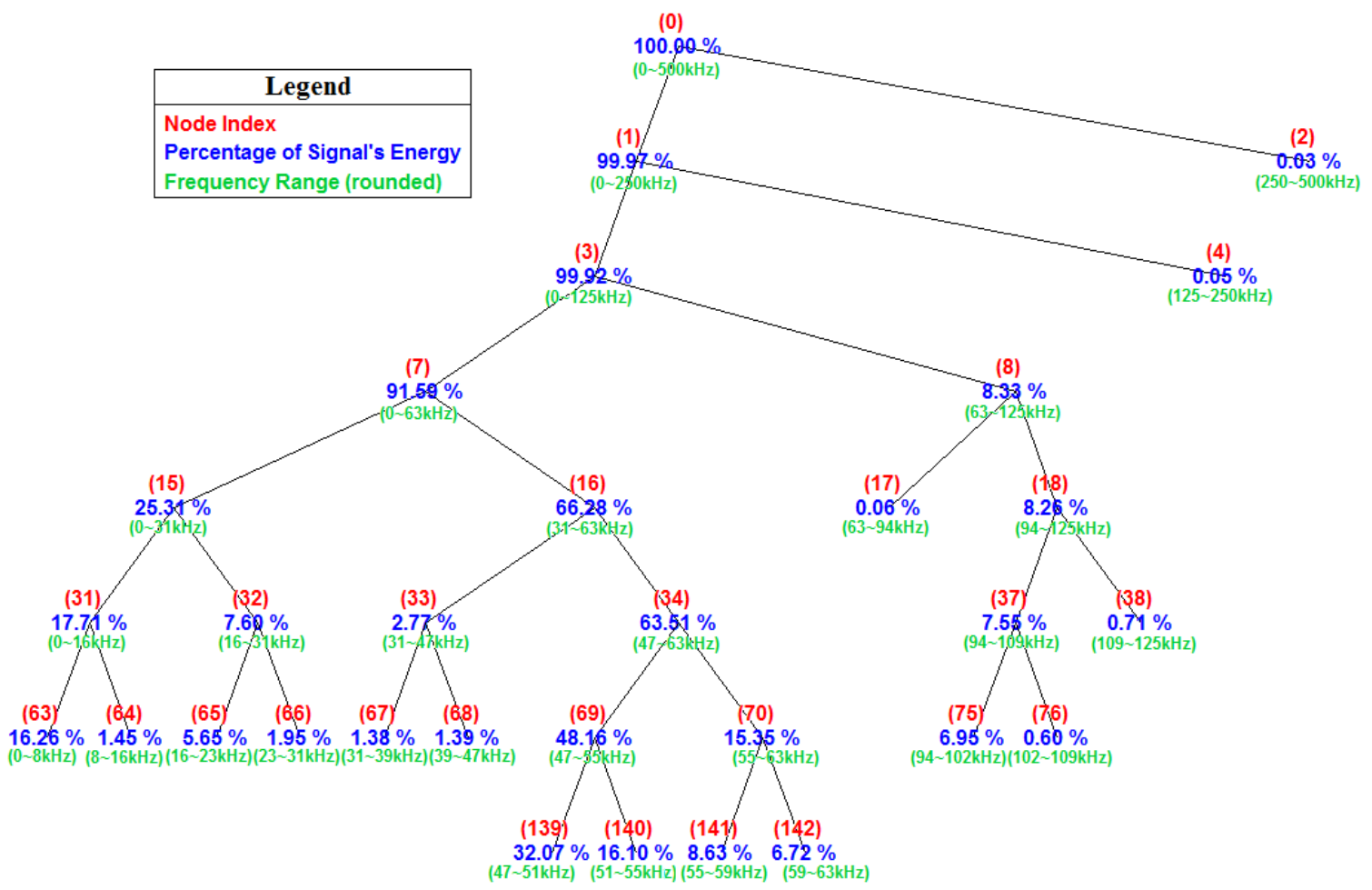

Fig. B.1. Each node is labeled with its index number, the percentage of the total energy in the signal it contains, and the frequency range (rounded to nearest integer for readability). 

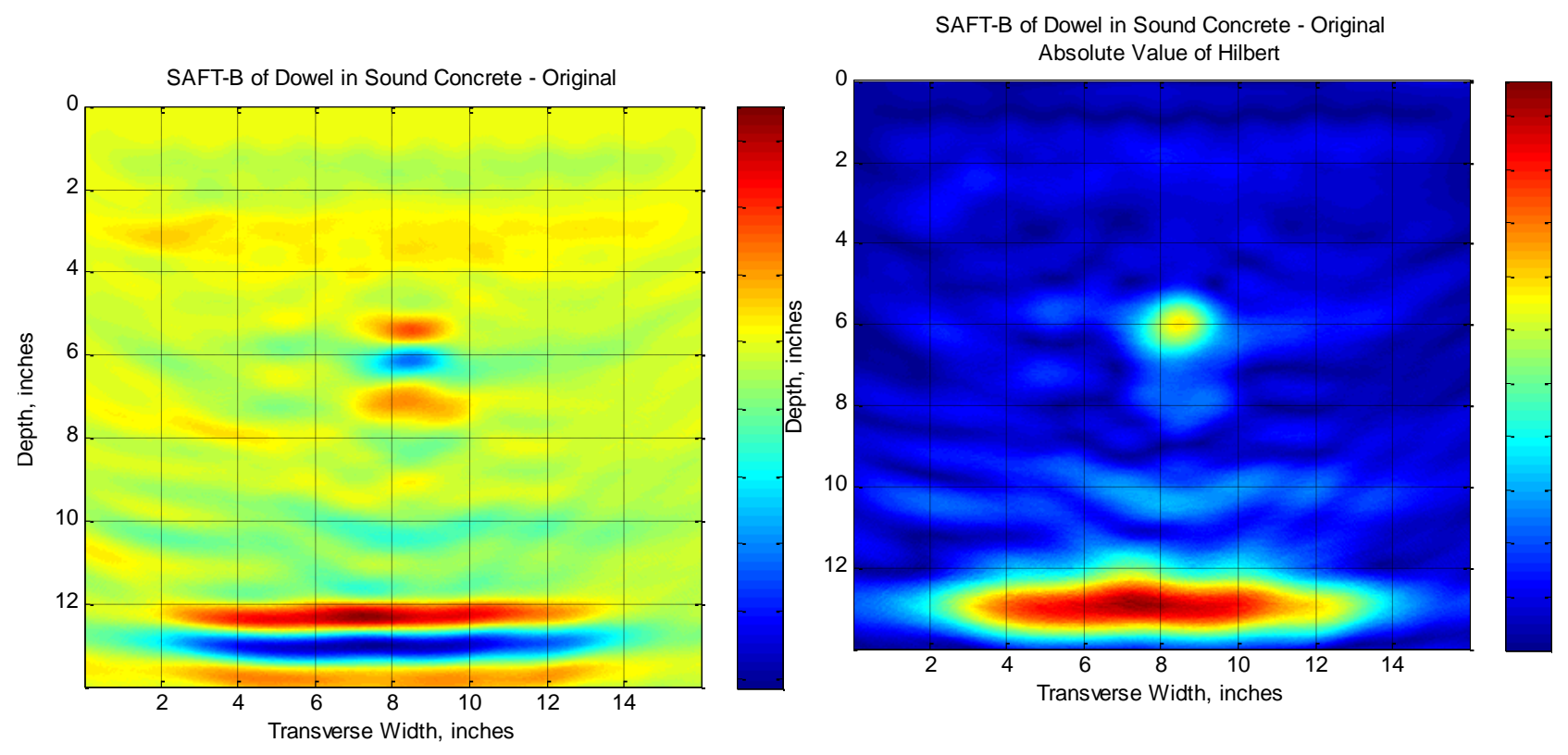

Fig. B.2. Data details - data: original; frequency range: $0 \sim 500 \mathrm{kHz}$; bandwidth: $500 \mathrm{kHz}$.
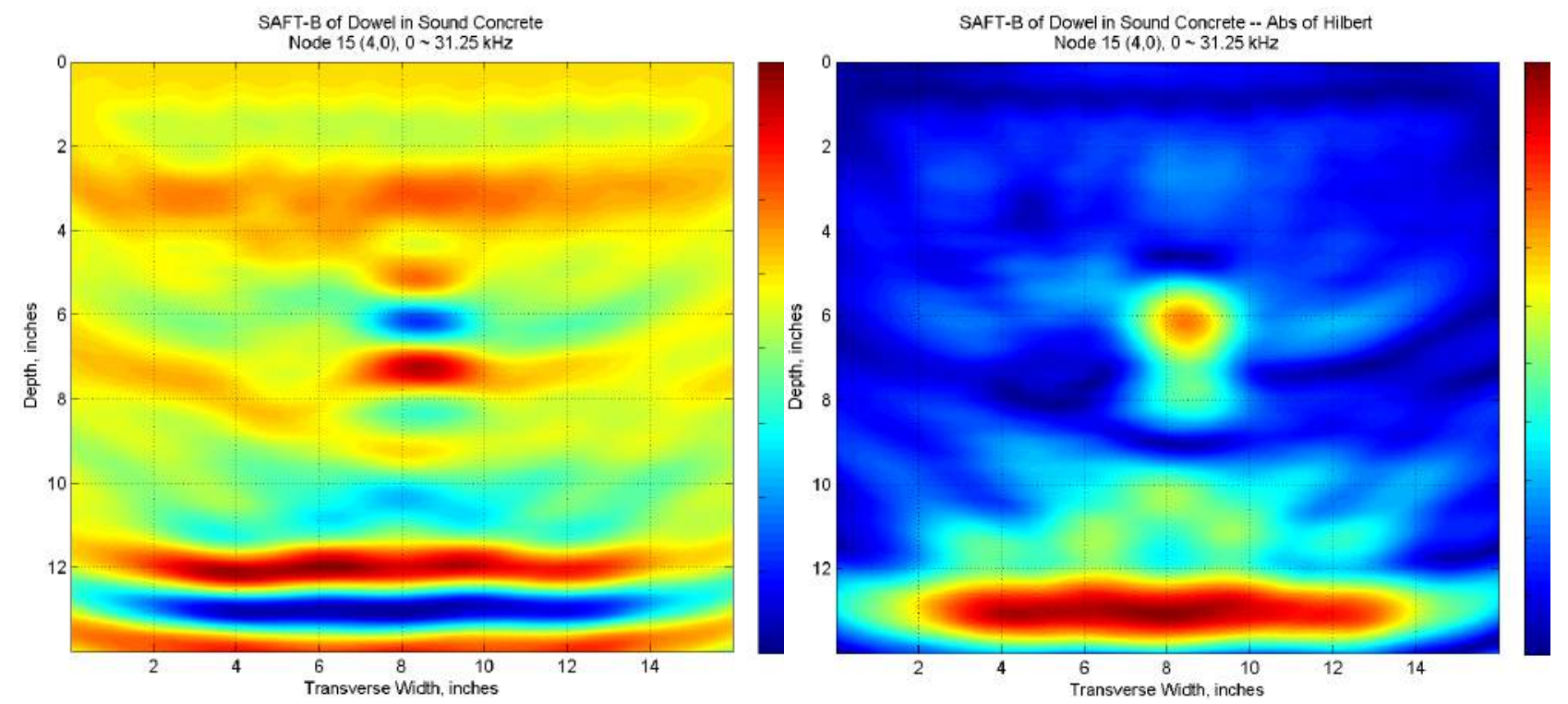

Fig. B.3. Data details - data: Node 15; frequency range: $0 \sim 31.25 \mathrm{kHz}$; bandwidth: $31.25 \mathrm{kHz}$. 

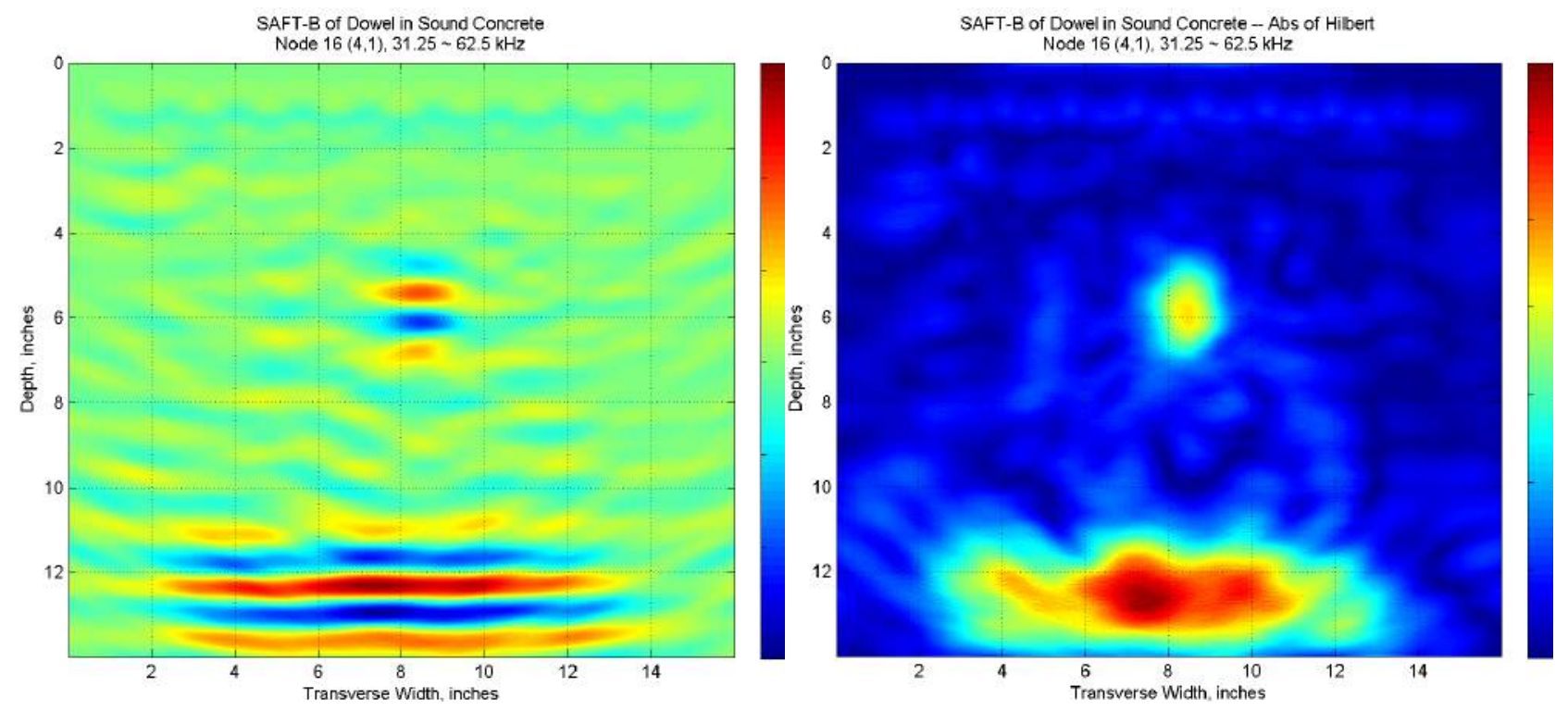

Fig. B.4. Data details - data: Node 16; frequency range: $31.25 \sim 62.5 \mathrm{kHz}$; bandwidth: $31.25 \mathrm{kHz}$.
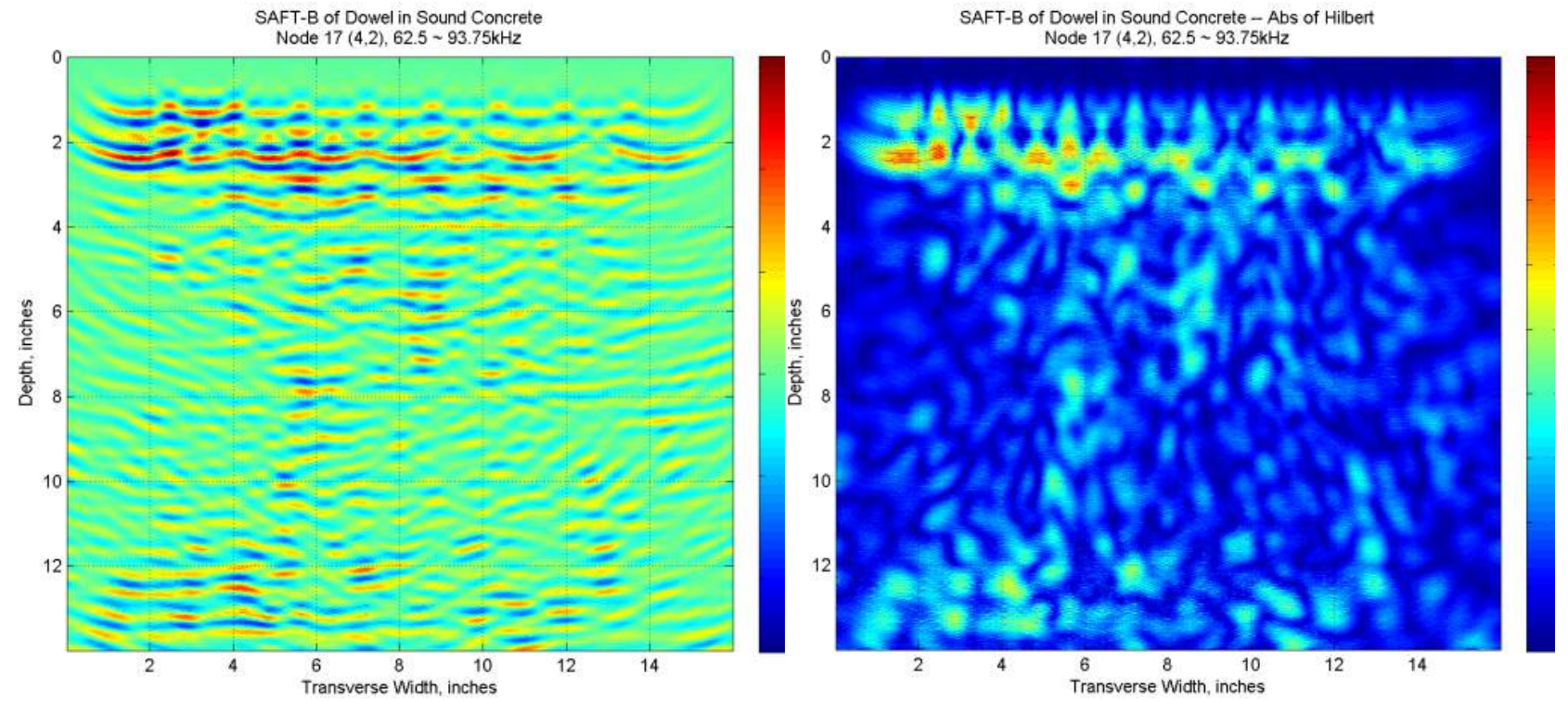

Fig. B.5. Data details - data: Node 17; frequency range: $62.5 \sim 93.75 \mathrm{kHz}$; bandwidth: $31.25 \mathrm{kHz}$. 

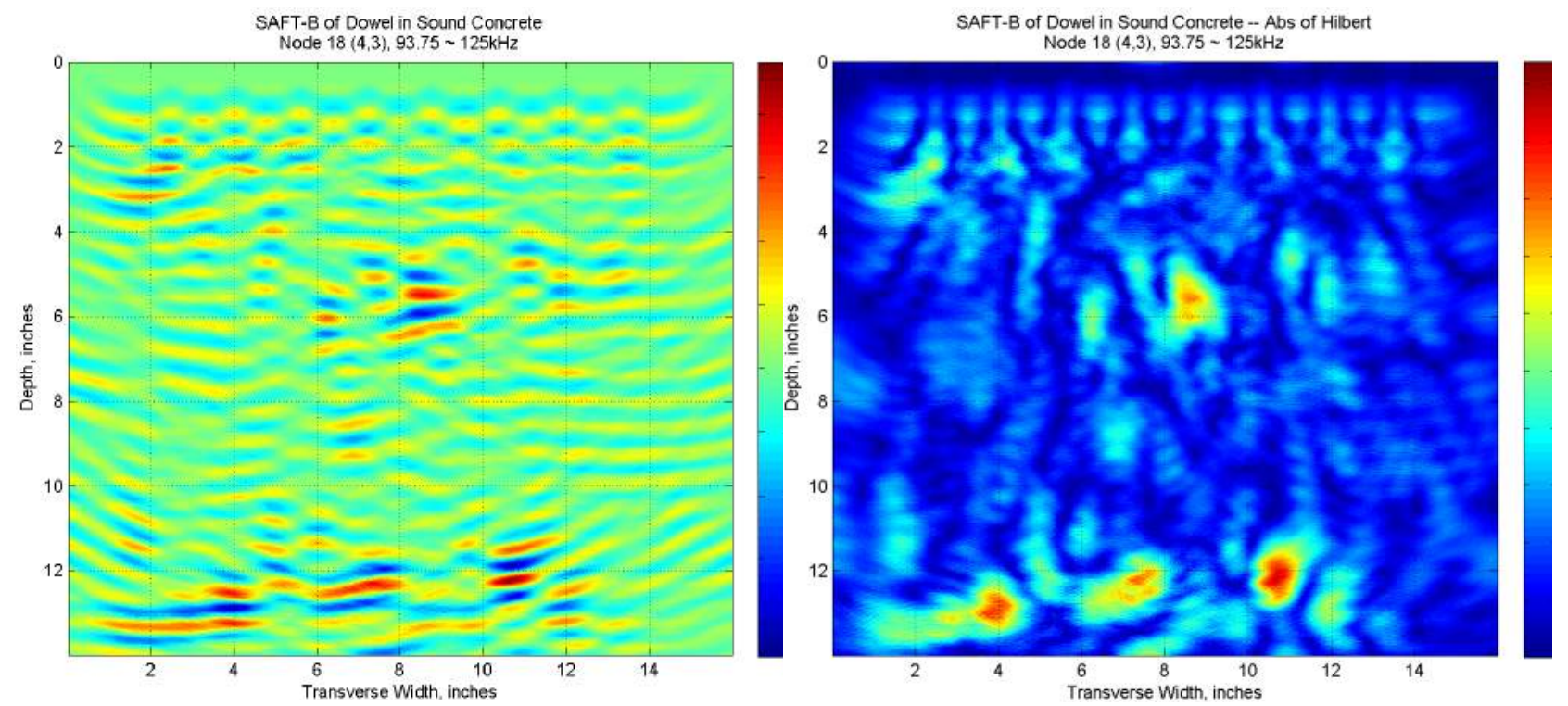

Fig. B.6. Data details - data: Node 18; frequency range: $93.75 \sim 125 \mathrm{kHz}$; bandwidth: $31.25 \mathrm{kHz}$.
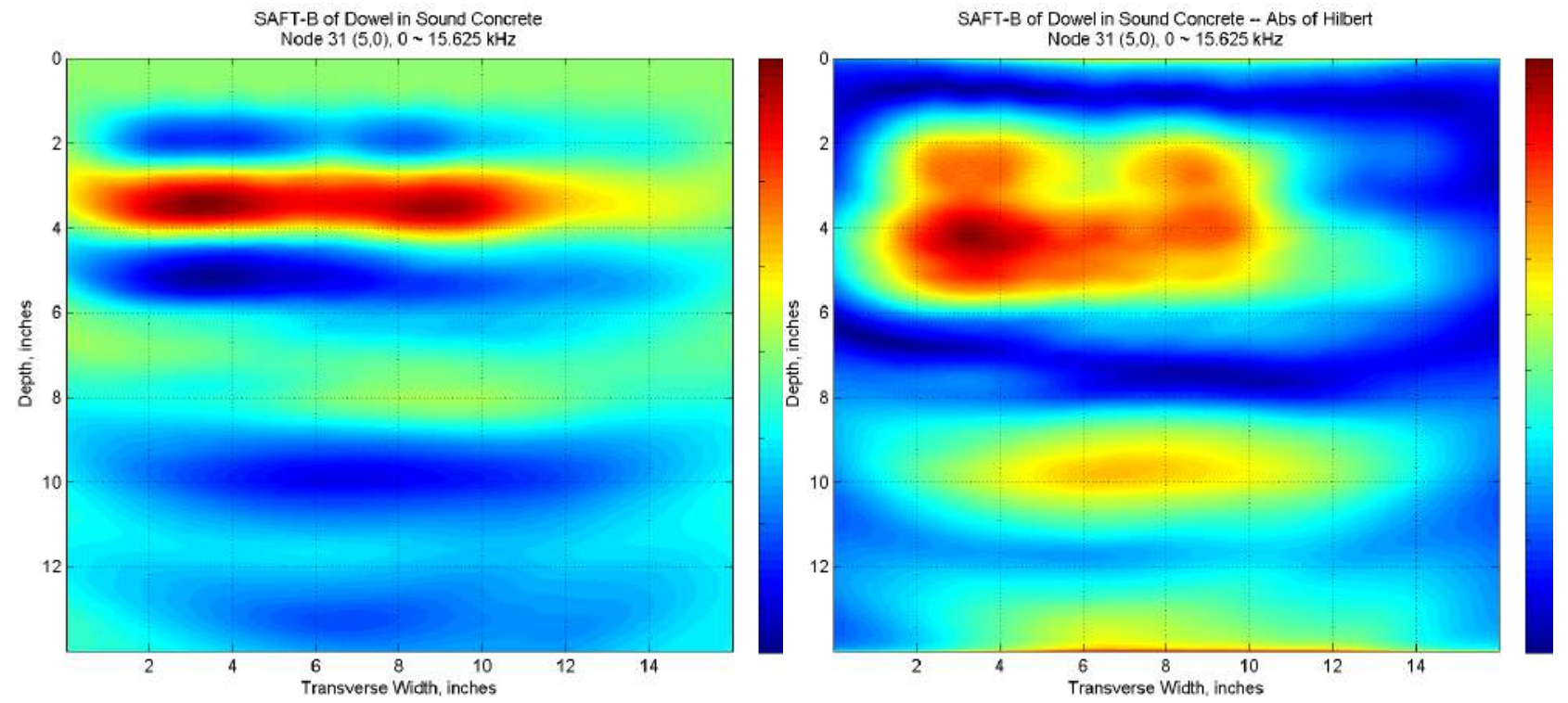

Fig. B.7. Data details - data: Node 31; frequency range: $0 \sim 15.625 \mathrm{kHz}$; bandwidth: $15.625 \mathrm{kHz}$. 

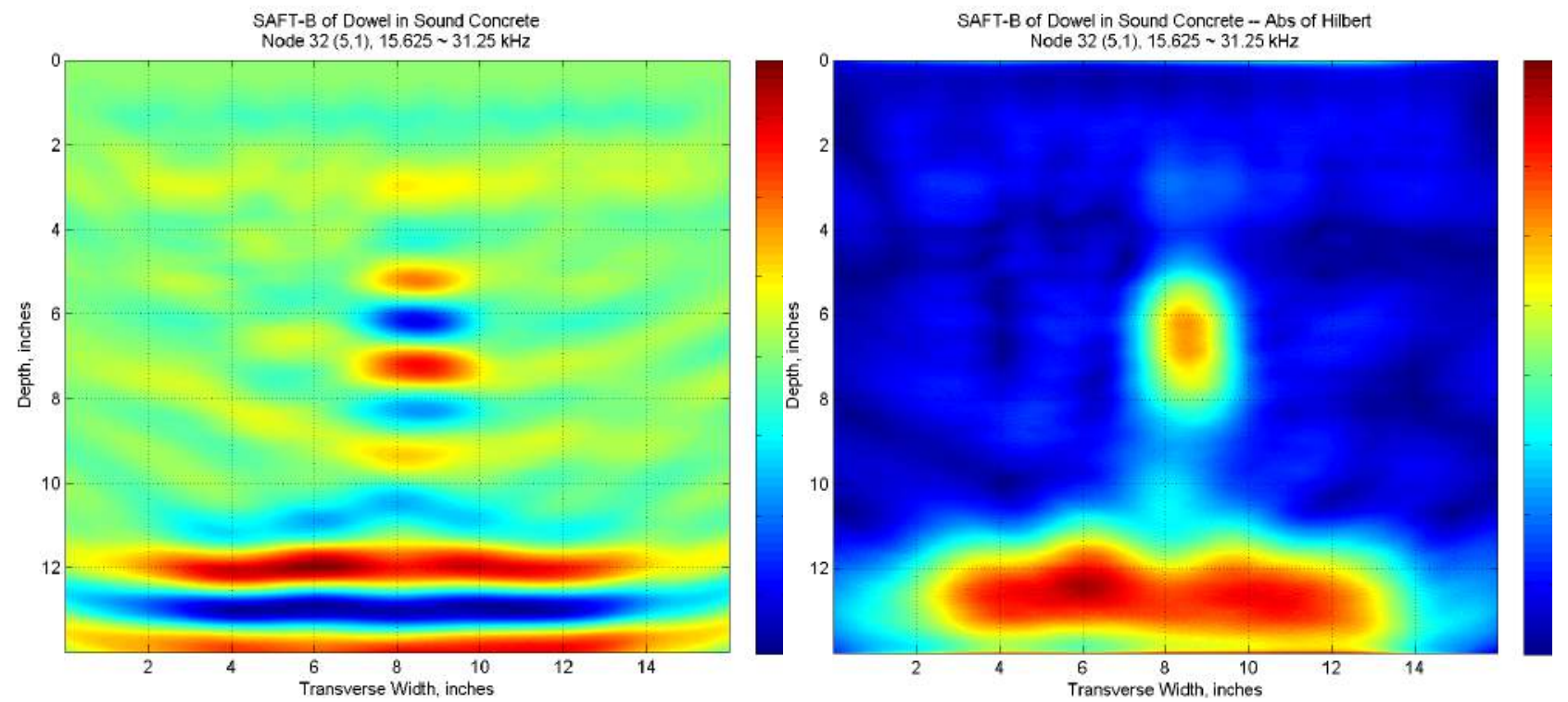

Fig. B.8. Data details - data: Node 32; frequency range: $15.625 \sim 31.25 \mathrm{kHz}$; bandwidth: $15.625 \mathrm{kHz}$.
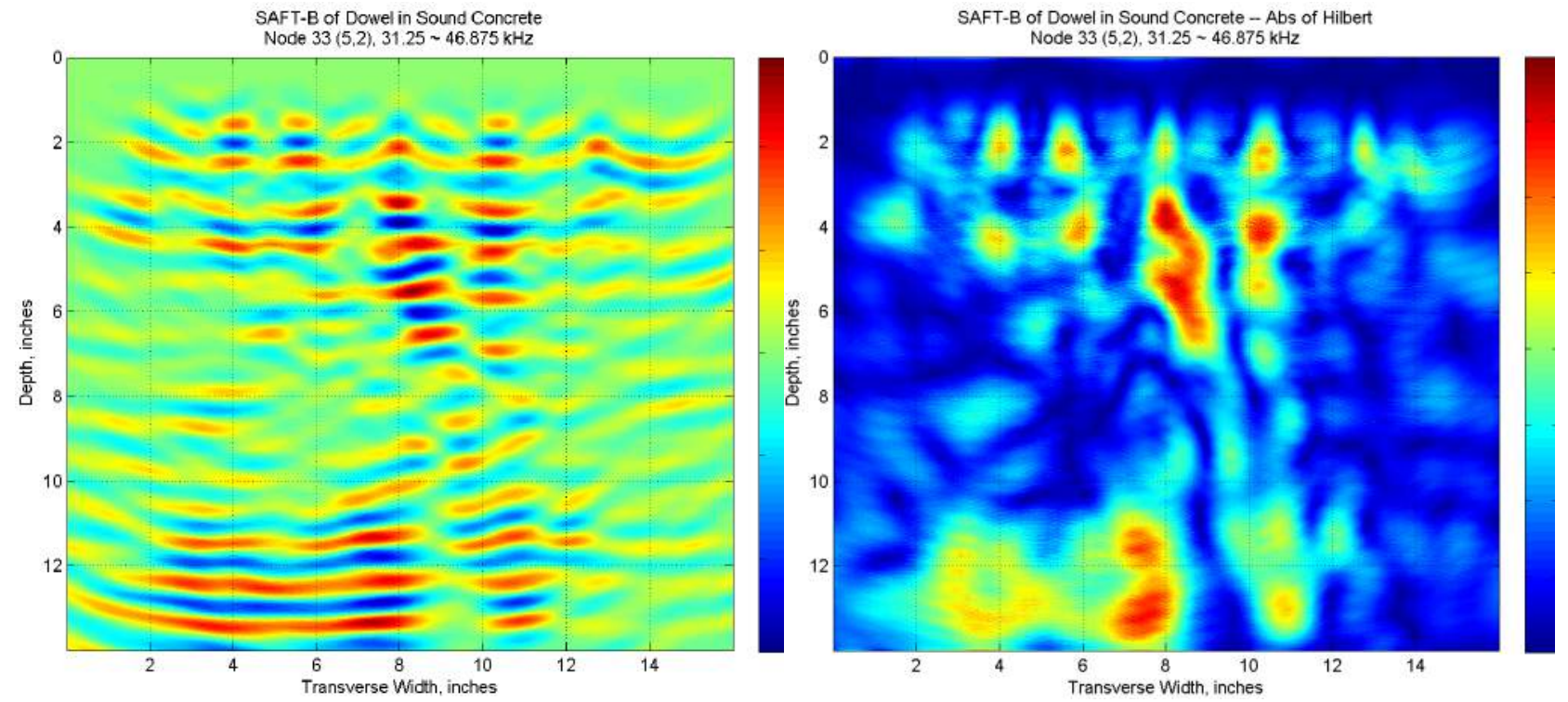

Fig. B.9. Data details - data: Node 33; frequency range: $31.25 \sim 46.875 \mathrm{kHz}$; bandwidth: $15.625 \mathrm{kHz}$. 

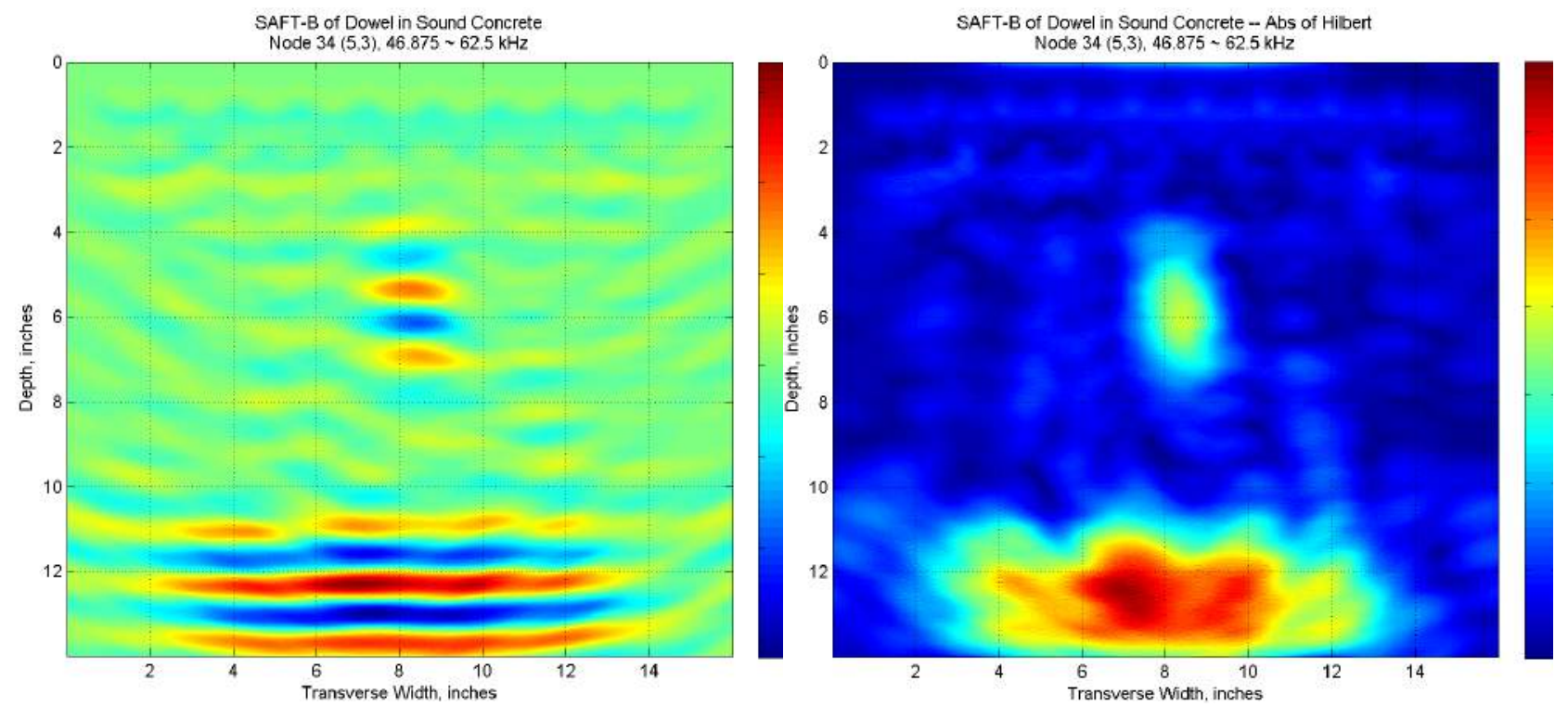

Fig. B.10. Data details - data: Node 34; frequency range: $46.875 \sim 62.5 \mathrm{kHz}$; bandwidth: $15.625 \mathrm{kHz}$.
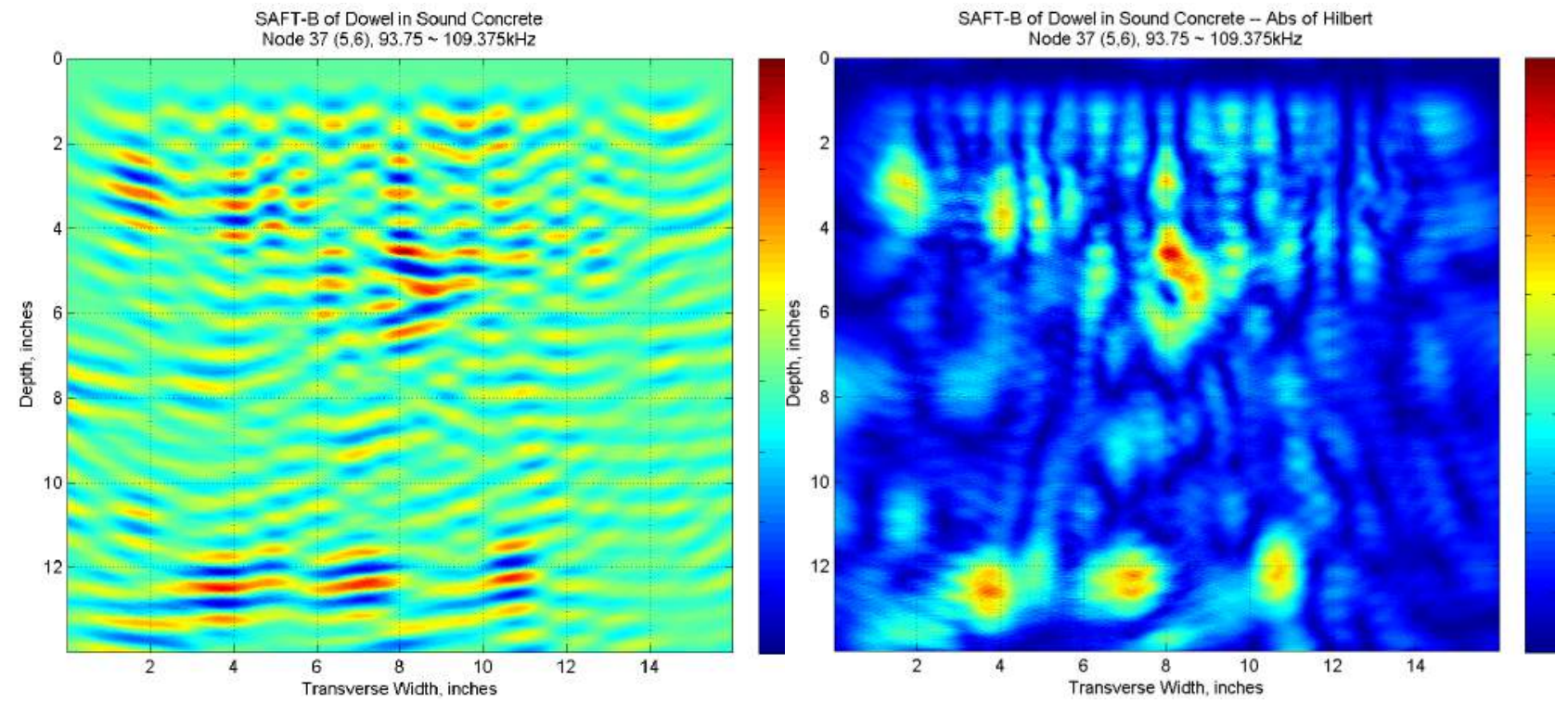

Fig. B.11. Data details - data: Node 37; frequency range: $93.75 \sim 109.375 \mathrm{kHz}$; bandwidth: $15.625 \mathrm{kHz}$. 

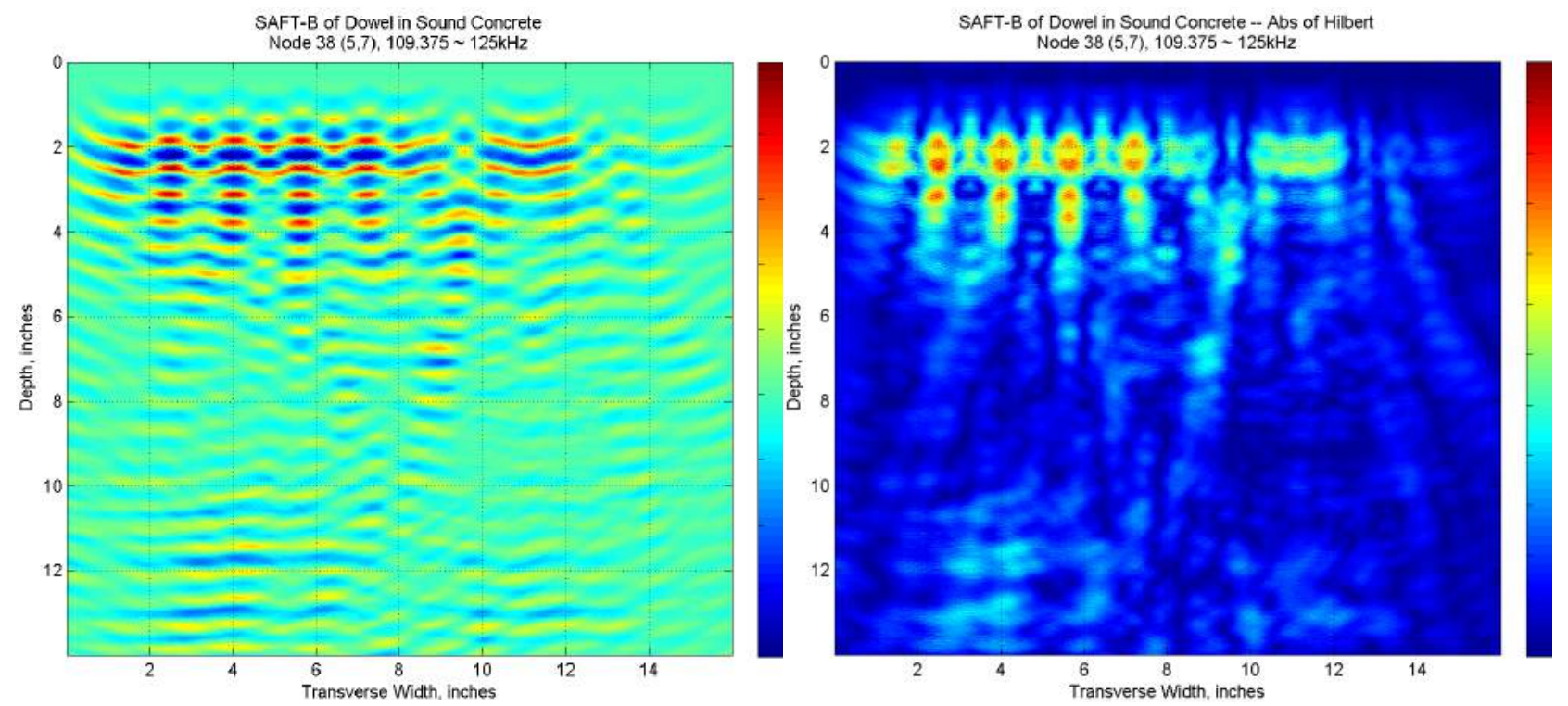

Fig. B.12. Data details - data: Node 38; frequency range: $109.375 \sim 125 \mathrm{kHz}$; bandwidth: $15.625 \mathrm{kHz}$.
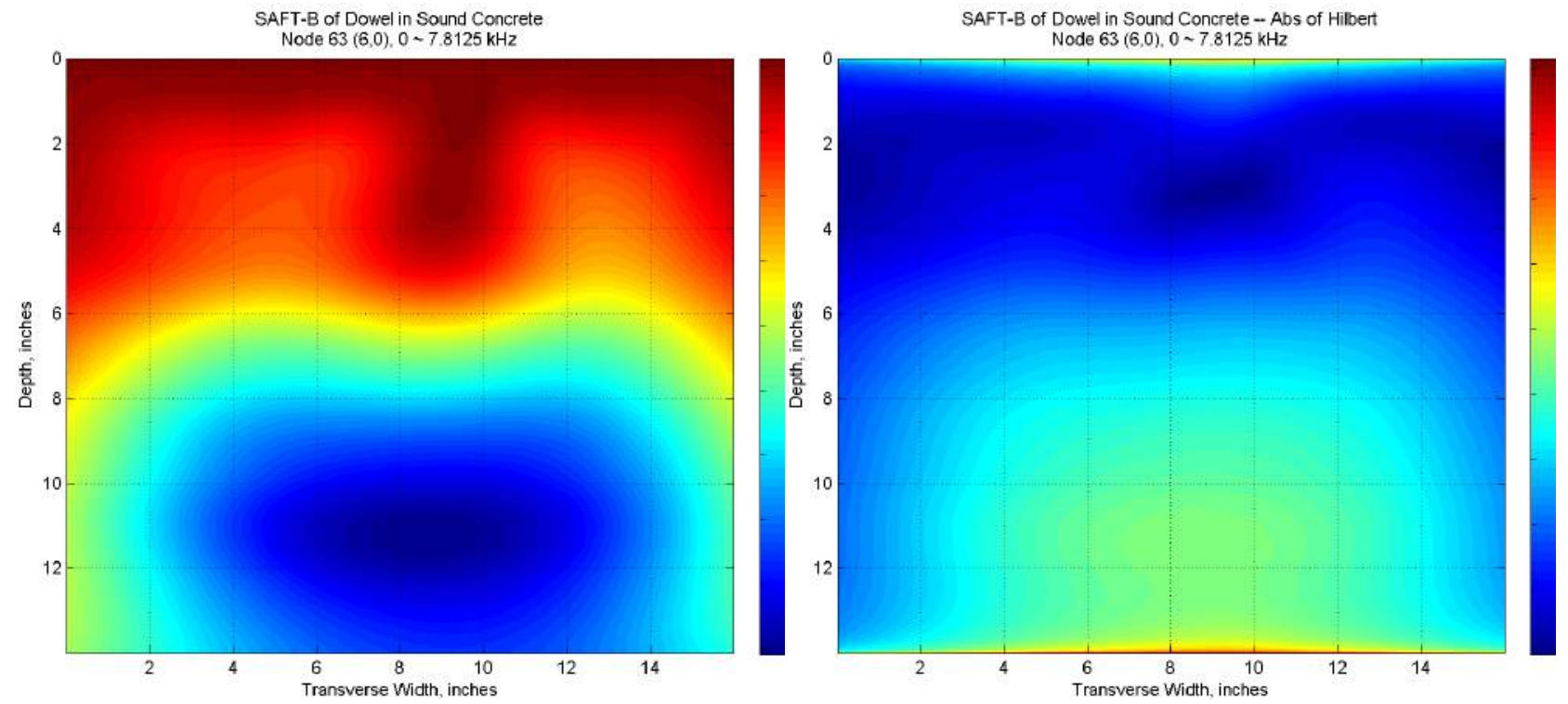

Fig. B.13. Data details - data: Node 63; frequency range: $0 \sim 7.8125 \mathrm{kHz}$; bandwidth: $7.8125 \mathrm{kHz}$. 

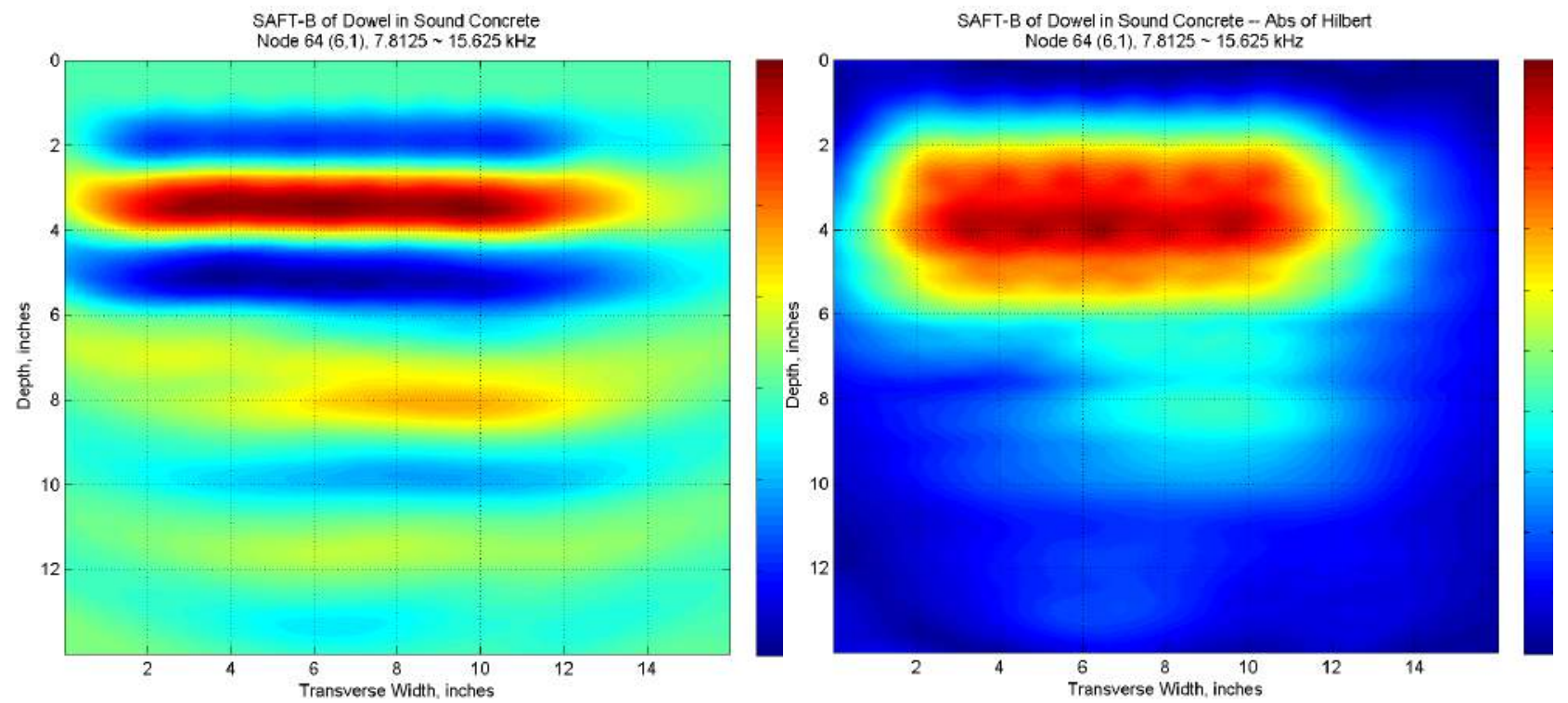

Fig. B.14. Data details - data: Node 64; frequency range: $7.8125 \sim 15.625 \mathrm{kHz}$; bandwidth: $7.8125 \mathrm{kHz}$.
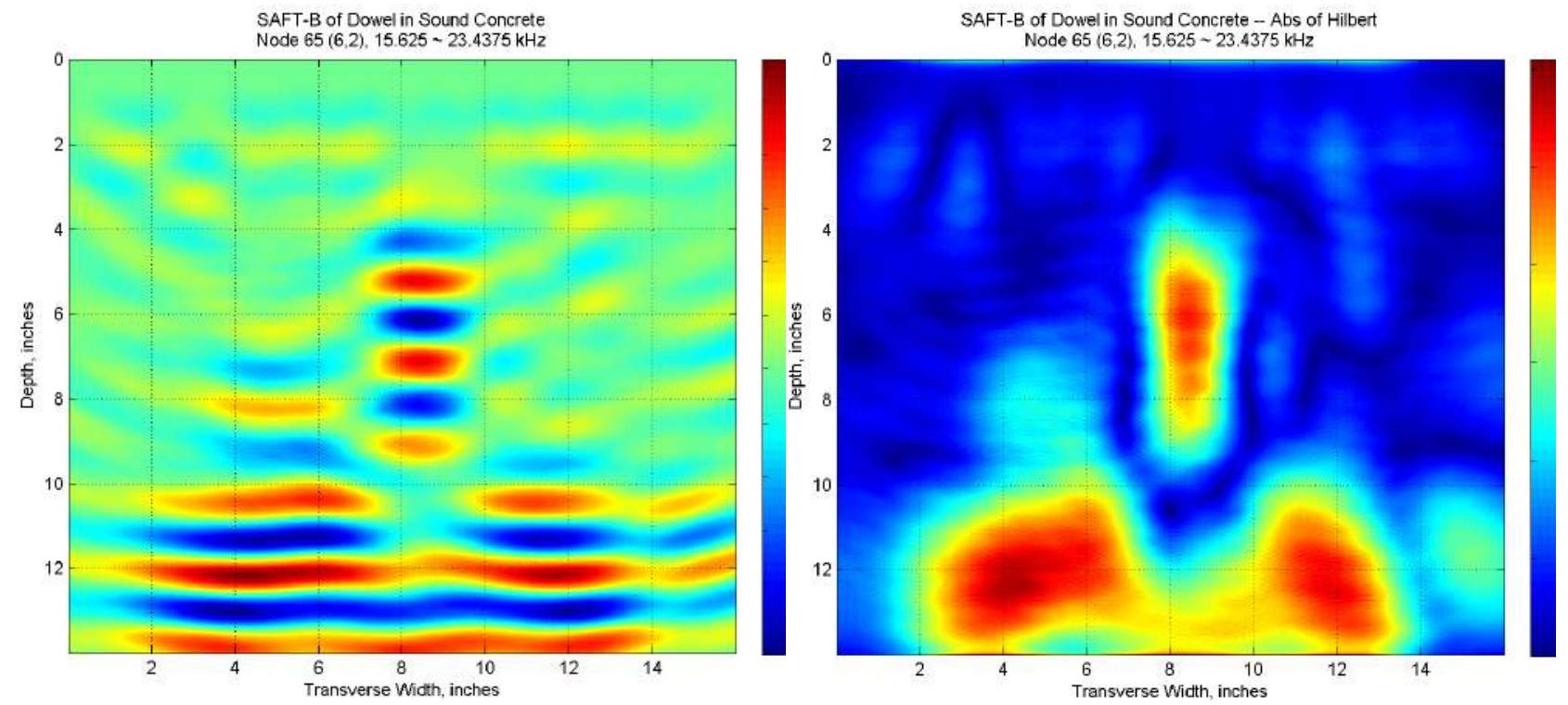

Fig. B.15. Data details - data: Node 65; frequency range: $15.625 \sim 23.4375 \mathrm{kHz}$; bandwidth: $7.8125 \mathrm{kHz}$. 

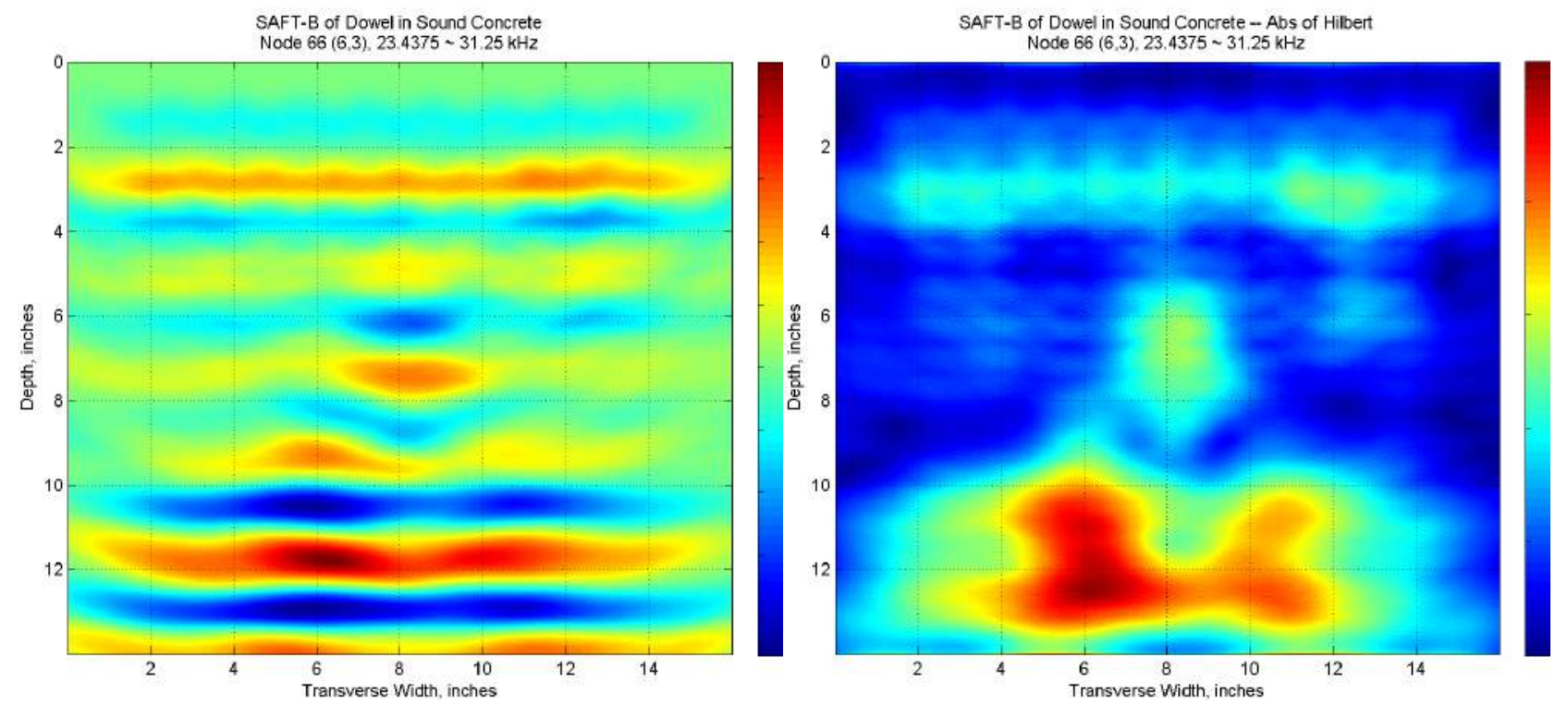

Fig. B.16. Data details - data: Node 66; frequency range: $23.4375 \sim 31.25 \mathrm{kHz}$; bandwidth: $7.8125 \mathrm{kHz}$.
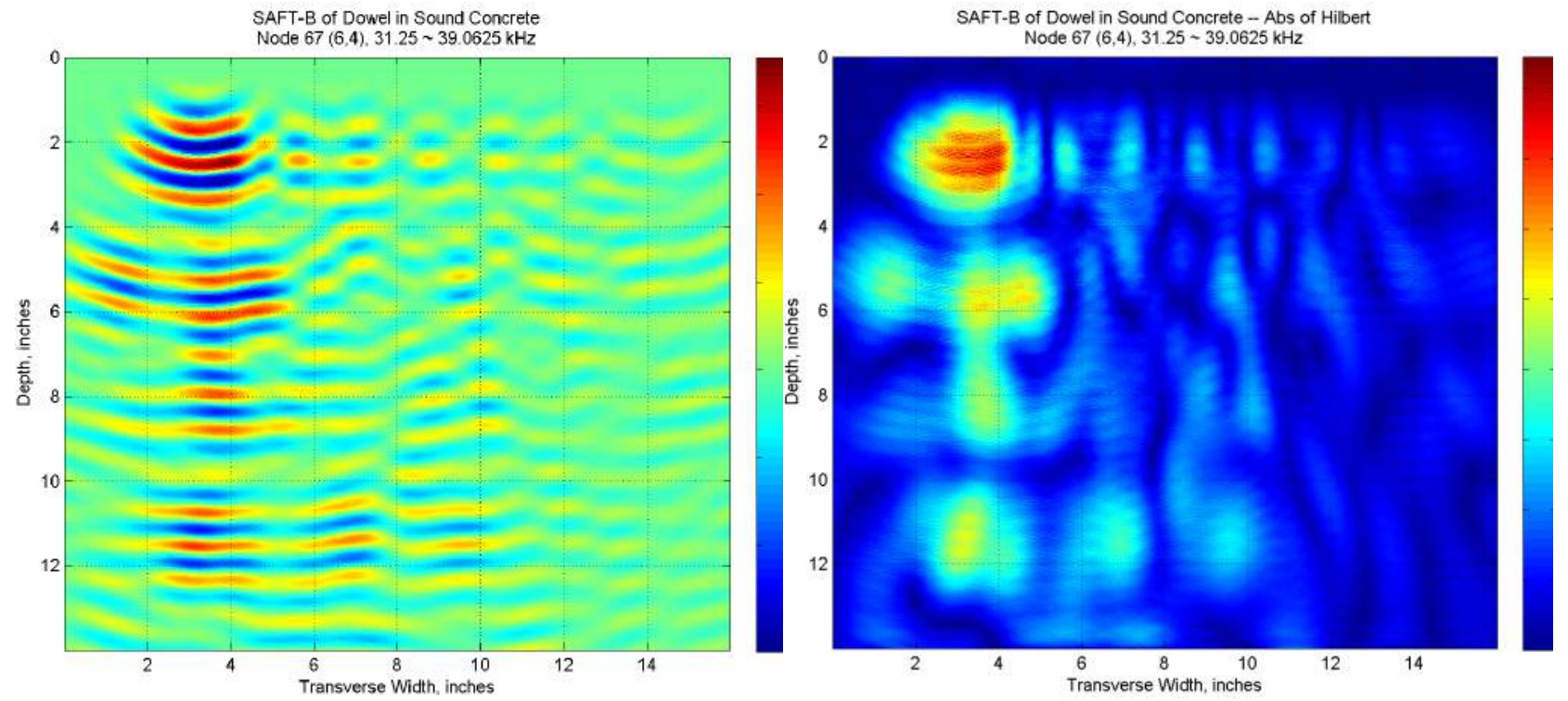

Fig. B.17. Data details - data: Node 67; frequency range: $31.25 \sim 39.0625 \mathrm{kHz}$; bandwidth: $7.8125 \mathrm{kHz}$. 

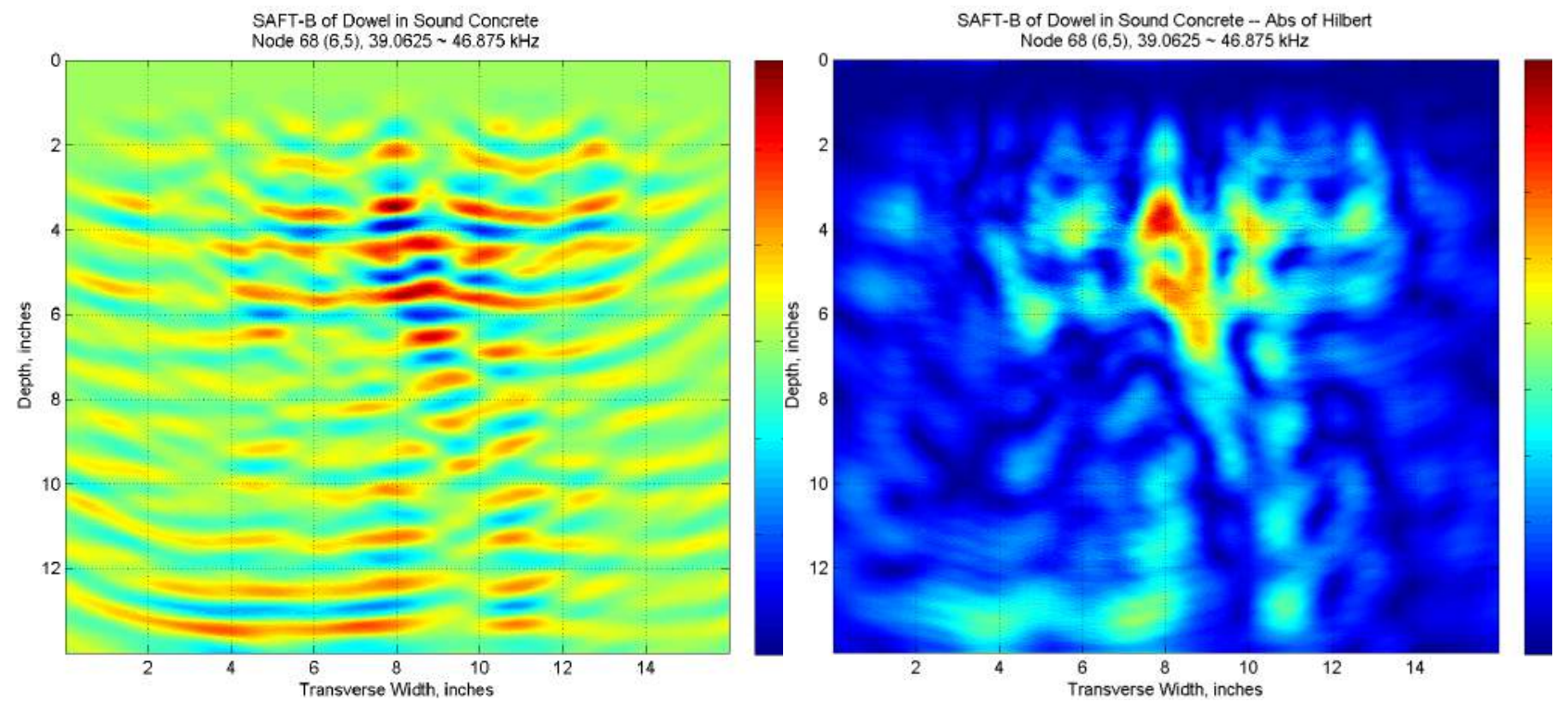

Fig. B.18. Data details - data: Node 68; frequency range: $39.0625 \sim 46.875 \mathrm{kHz}$; bandwidth: $7.8125 \mathrm{kHz}$.
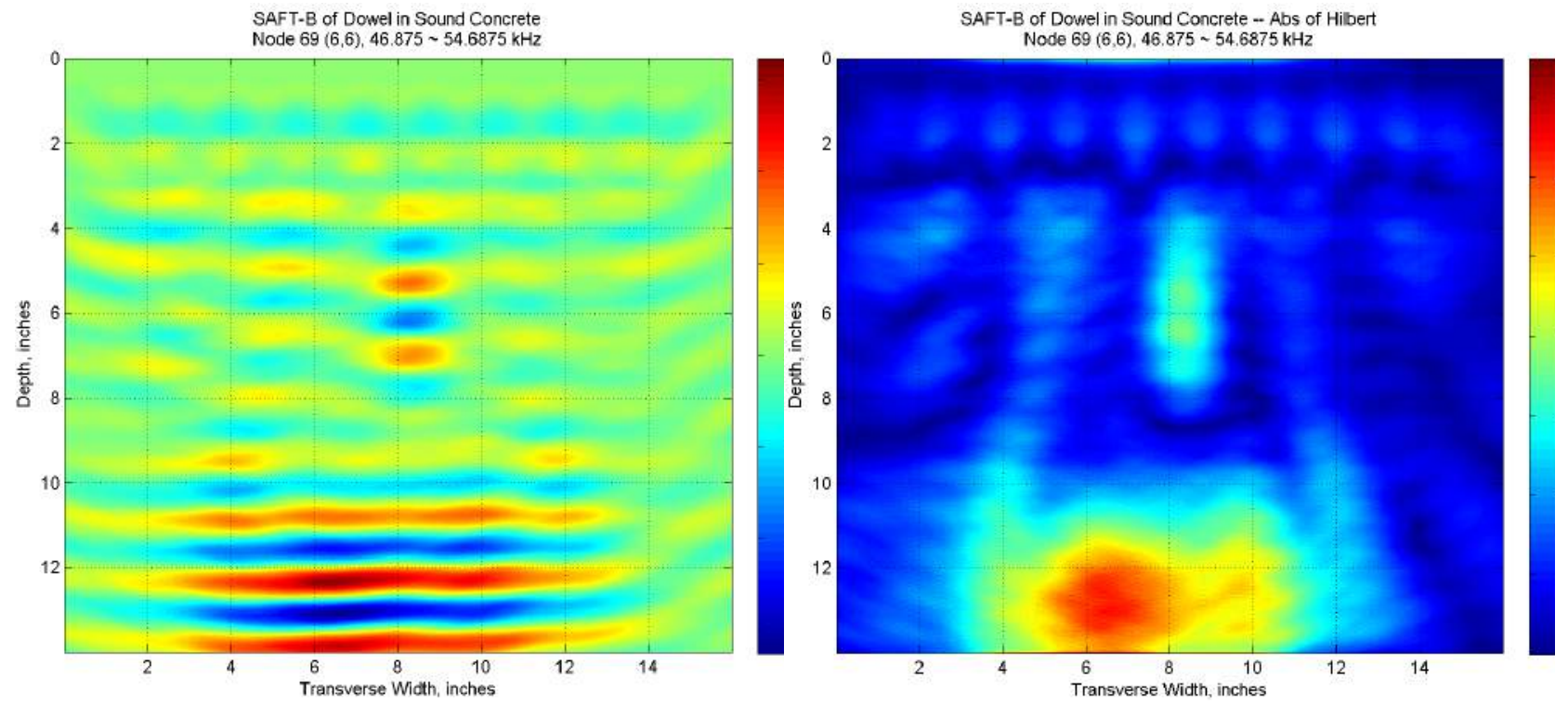

Fig. B.19. Data details - data: Node 69; frequency range: $46.875 \sim 54.6875 \mathrm{kHz}$; bandwidth: $7.8125 \mathrm{kHz}$. 

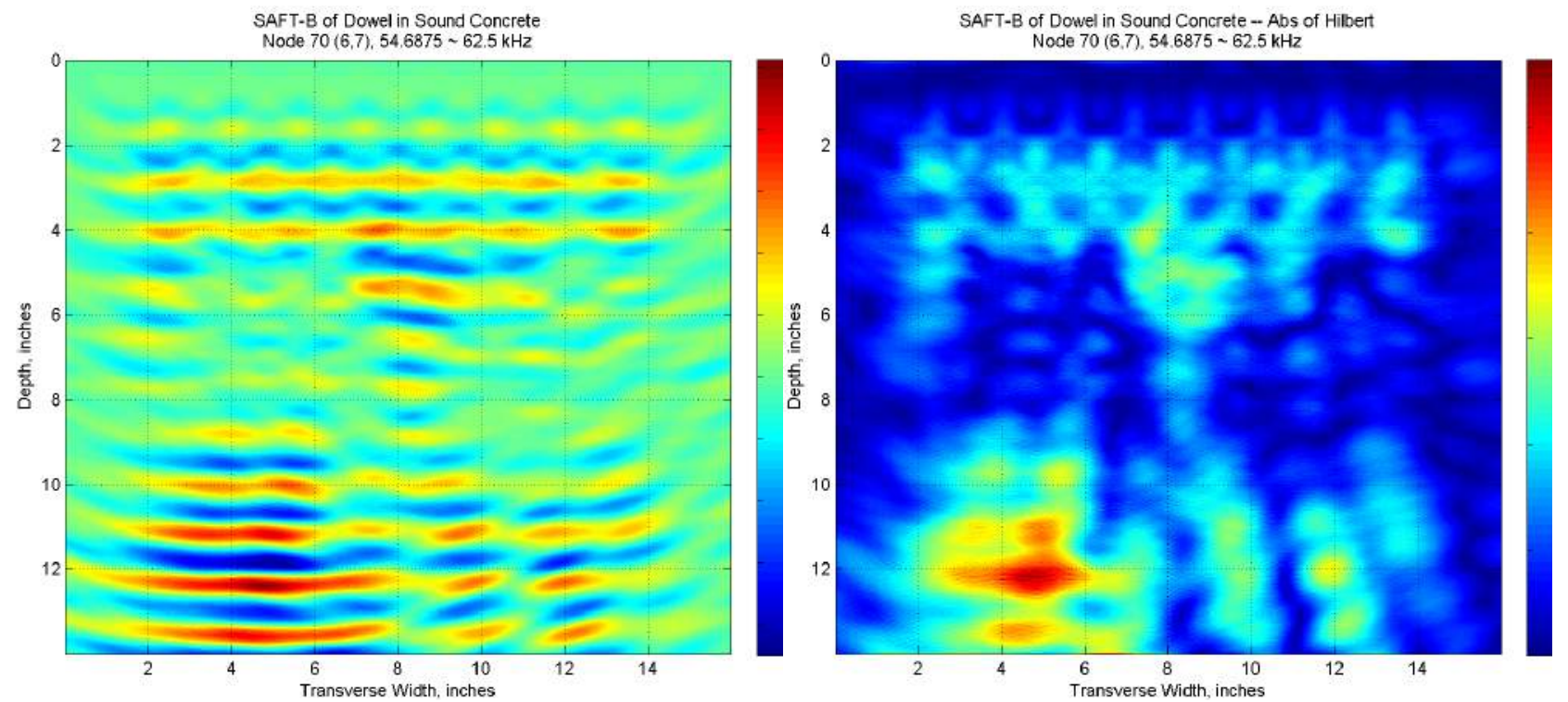

Fig. B.20. Data details - data: Node 70; frequency range: $54.6875 \sim 62.5 \mathrm{kHz}$; bandwidth: $7.8125 \mathrm{kHz}$.
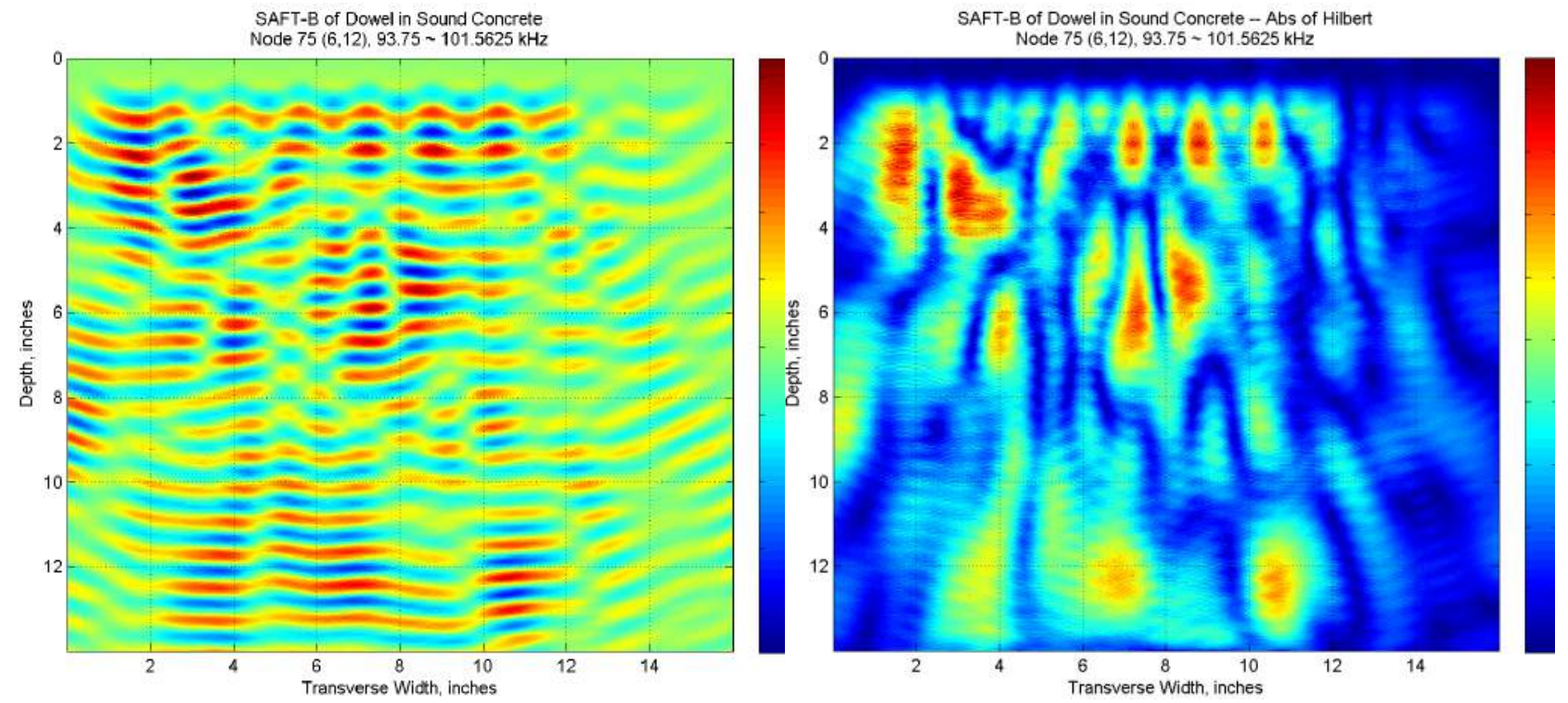

Fig. B.21. Data details - data: Node 75; frequency range: $93.75 \sim 101.5625 \mathrm{kHz}$; bandwidth: $7.8125 \mathrm{kHz}$. 

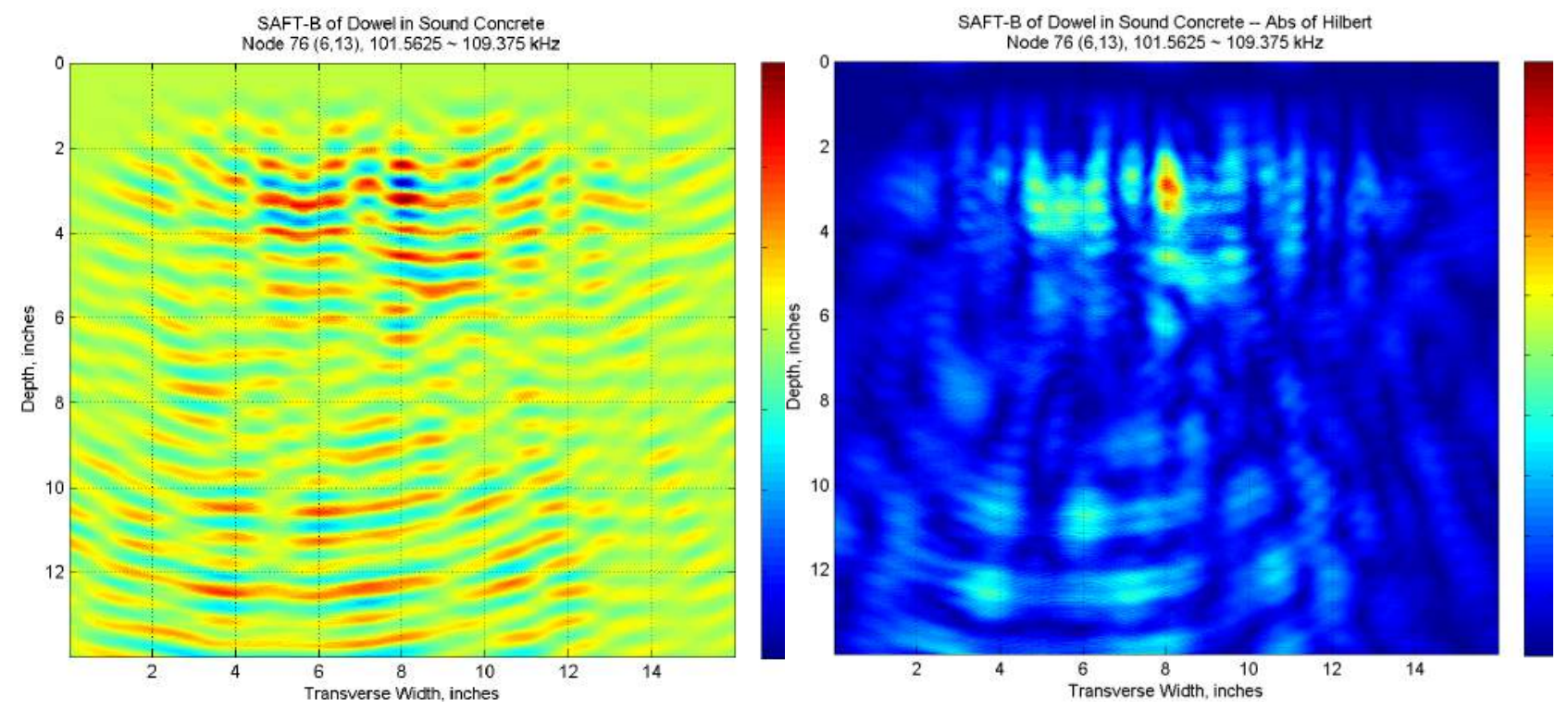

Fig. B.22. Data details - data: Node 76; frequency range: $101.5625 \sim 109.375 \mathrm{kHz}$; bandwidth: $7.8125 \mathrm{kHz}$.
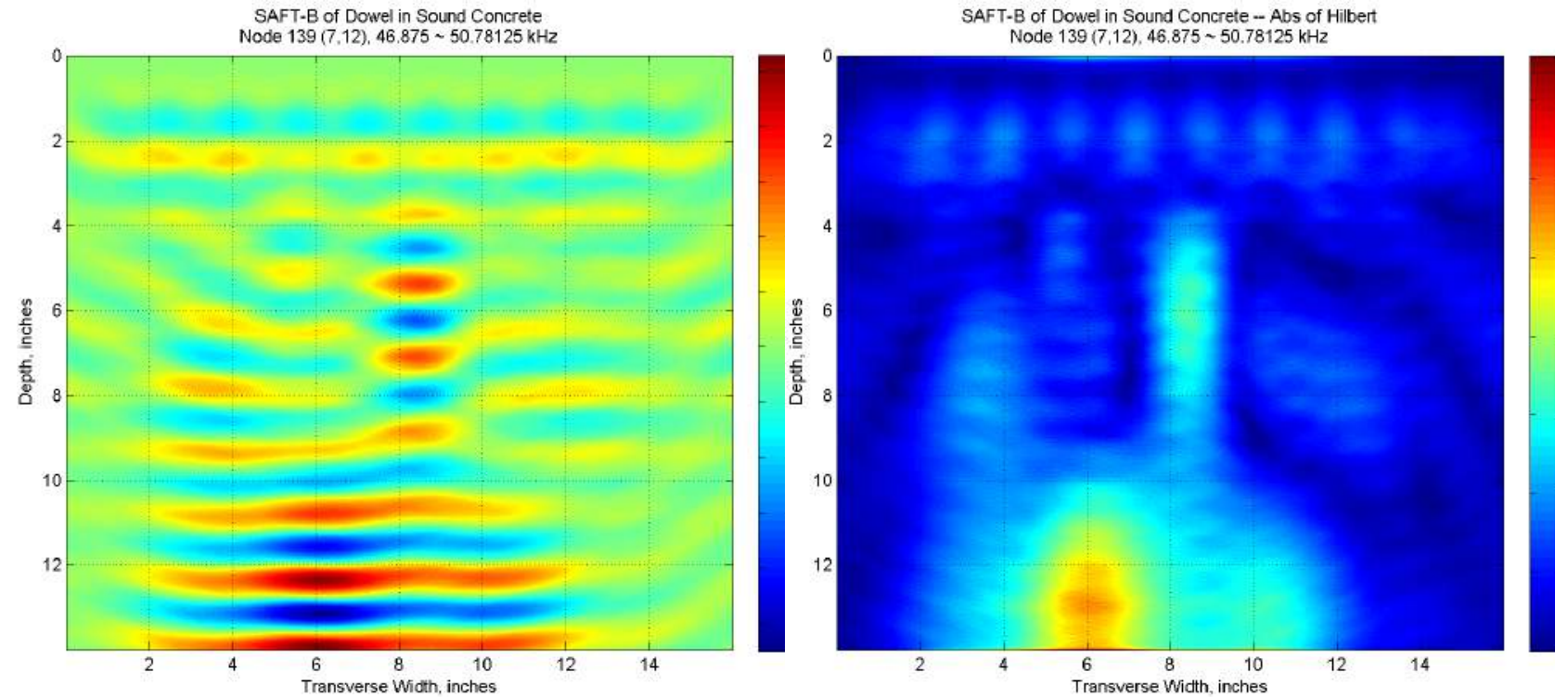

Fig. B.23. Data details - data: Node 139; frequency range: $46.875 \sim 50.78125 \mathrm{kHz}$; bandwidth: $3.90625 \mathrm{kHz}$. 

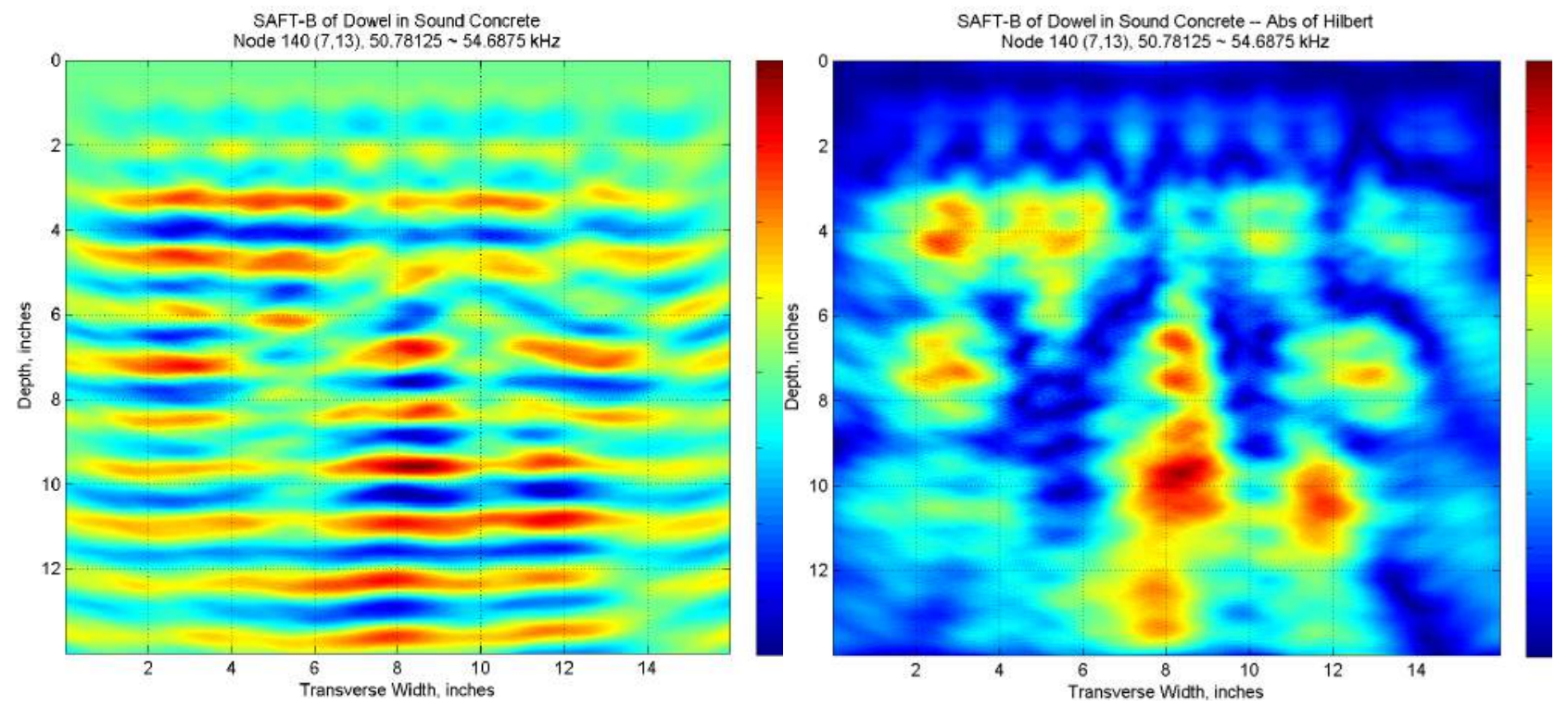

Fig. B.24. Data details - data: Node 140; frequency range: $50.78125 \sim 54.6875 \mathrm{kHz}$; bandwidth: $3.90625 \mathrm{kHz}$.
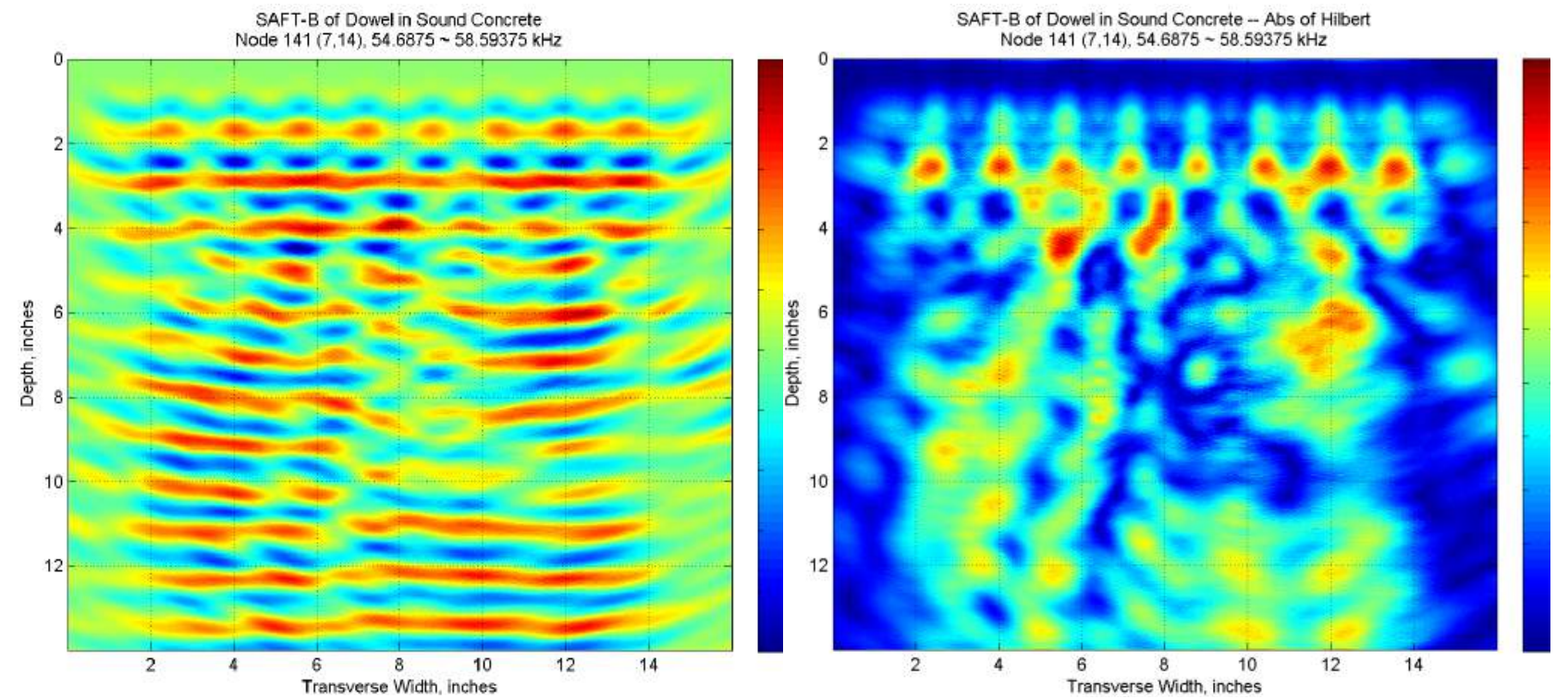

Fig. B.25. Data details - data: Node 141; frequency range: $54.6875 \sim 58.59375 \mathrm{kHz}$; bandwidth: $3.90625 \mathrm{kHz}$. 

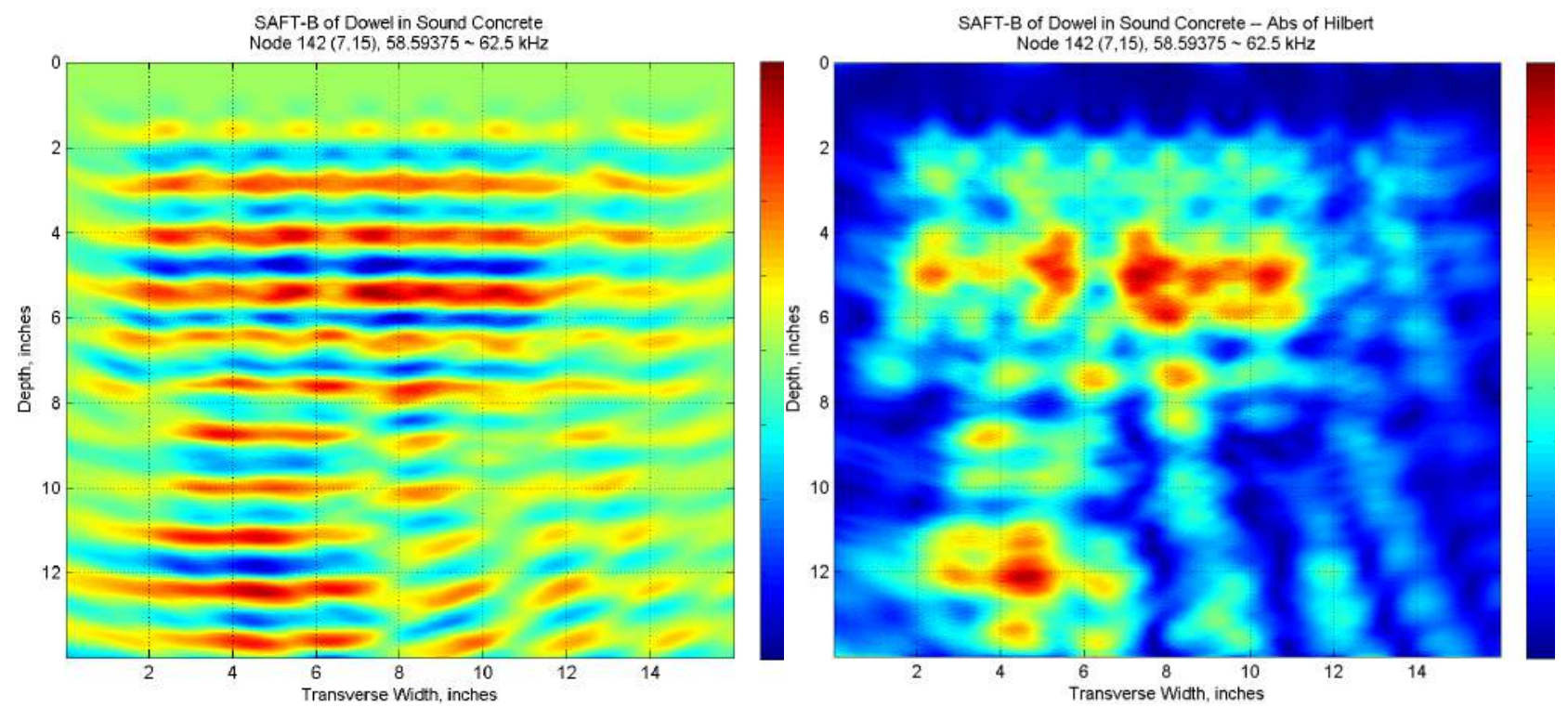

Fig. B.26. Data details - data: Node 142; frequency range: $58.59375 \sim 62.5 \mathrm{kHz}$; bandwidth: $3.90625 \mathrm{kHz}$. 